\title{
Medium-resolution échelle spectroscopy of the Red Square Nebula, MWC 922^
}

\author{
N. Wehres ${ }^{1}$, B. B. Ochsendorf ${ }^{2}$, A. G. G. M. Tielens ${ }^{3}$, N. L. J. Cox ${ }^{4,5}$, L. Kaper ${ }^{6}$, J. Bally ${ }^{7}$, and T. P. Snow ${ }^{7}$ \\ 1 I. Physikalisches Institut, Universität zu Köln, Zülpicher Str. 77, 50937 Köln, Germany \\ e-mail: wehres@ph1.uni-koeln.de \\ 2 Department of Physics and Astronomy, The Johns Hopkins University, 3400 North Charles Street, Baltimore, MD 21218, USA \\ 3 Leiden Observatory, Leiden University, PO Box 9513, 2300 RA Leiden, The Netherlands \\ ${ }^{4}$ Université de Toulouse, UPS-OMP, Institut de Recherche en Astrophysique et Planétologie, 31400 Toulouse, France \\ 5 CNRS, IRAP, 9 Av. Colonel Roche, BP 44346, 31028 Toulouse Cedex 4, France \\ 6 Department voor Sterrenkunde, Universiteit van Amsterdam, The Netherlands \\ 7 Department for Astronomy and Space Sciences, University of Colorado, Boulder, CO 80304, USA
}

Received 13 April 2016 / Accepted 5 March 2017

\begin{abstract}
Context. Medium-resolution échelle spectra of the Red Square Nebula surrounding the star MWC 922 are presented. The spectra have been obtained in 2010 and 2012 using the X-shooter spectrograph mounted on the Very Large Telescope (VLT) in Paranal, Chile. The spectrum covers a wavelength range between $300 \mathrm{~nm}-2.5 \mu \mathrm{m}$ and shows that the nebula is rich in emission lines.

Aims. We aim to identify the emission lines and use them as a tool to determine the physical and chemical characteristics of the nebula. The emission lines are also used to put constraints on the structure of the nebula and on the nature of the central stars.

Methods. We analyzed and identified emission lines that indicated that the Red Square Nebula consists of a low density bipolar outflow, eminent in the broad emission component seen in [Fe II], as well as in P Cygni line profiles indicative of fast outflowing material. The narrow component in the [Fe II] lines is most likely formed in the photosphere of a surrounding disk. Some of the emission lines show a pronounced double peaked profile, such as Ca II, indicating an accretion disk in Keplerian rotation around the central star. [O I] emission lines are formed in the neutral atomic zone separating the ionized disk photosphere from the molecular gas in the interior of the disk, which is prominent in molecular $\mathrm{CO}$ emission in the near-IR. [N II] and [S II] emission clearly originates in a low density but fairly hot $(7000-10000 \mathrm{~K})$ nebular environment. H I recombination lines trace the extended nebula as well as the photosphere of the disk.

Results. These findings put constraints on the evolution of the central objects in MWC 922. The Red Square shows strong similarities to the Red Rectangle Nebula, both in morphology and in its mid-IR spectroscopic characteristics. As for the Red Rectangle, the observed morphology of the nebula reflects mass-loss in a binary system. Specifically, we attribute the biconical morphology and the associated rung-like structure to the action of intermittent jets blown by the accreting companion in a dense shell, which has been created by the primary. We stress, though, that despite the morphological similarities, these two objects represent very different classes of stellar objects.
\end{abstract}

Key words. astrochemistry - methods: observational - techniques: spectroscopic - stars: emission-line, $\mathrm{Be}-$ circumstellar matter - ISM: abundances

\section{Introduction}

The source MWC 922 is a B[e] emission line star in the center of the Red Square Nebula (IRAS 18184-1302). In Lamers et al. (1998) B[e] stars were classified into different groups, whereas MWC 922 remained in a group of unclassified B[e] stars. In Miroshnichenko (2007) a new classification of so-called FS CMa stars was introduced and MWC 922 was eventually classified within the group of FS CMa stars, exhibiting many atomic and ionic emission lines in the optical spectrum, as well as warm dust emission and molecular gas in the near- and mid-IR. The classification of many $\mathrm{B}[\mathrm{e}]$ stars is uncertain, because of the poorly understood distance and hence luminosity. The evolutionary status of the Red Square Nebula is also unclear and both, preand post- main sequence status, have been suggested (The et al. 1994; Voors 1999).

\footnotetext{
* The data-reduced spectra are only available at the CDS via anonymous ftp to cdsarc.u-strasbg. fr (130.79.128.5) or via http://cdsarc.u-strasbg.fr/viz-bin/qcat?J/A+A/601/A69
}

MWC 922 has been studied spectroscopically before, mainly in the mid to near IR (Andrillat \& Swings 1976; Rudy et al. 1992; Molster et al. 2002a,b,c; Peeters et al. 2002). Molster et al. (2002a,b,c) and Peeters et al. (2002) used the Short and Long Wavelengths Spectrographs on board of the Infrared Space Observatory (ISO) in order to study the crystalline silicates and PAHs (polycyclic aromatic hydrocarbons) in a sample of evolved stars. Both emission features, crystalline silicates and PAHs, have been detected around MWC 922. In addition, the near-IR spectrum of MWC 922 reveals a plethora of atomic emission lines. Rudy et al. (1992) remarked the exceptional strength of the [Fe II] lines in this object.

Photometrically, the object was studied using narrow band $\mathrm{H} \alpha$ images by Marston \& McCollum (2008), specifically looking at extended shells in $\mathrm{B}[\mathrm{e}]$ stars. The same study mentions jet-like structures in MWC 922, extending from the south-east to the north-west. This is consistent with a recent study by Tuthill \& Lloyd (2007), who resolved the nebula structure using the $H$-band filter between $1.487-1.783 \mu \mathrm{m}$. In this study the 
morphology of the Red Square Nebula is compared to the Red Rectangle Nebula, both of which exhibit bi-polar outflows showing a so-called ladder-rung structure. The ladder-rungs have been associated with episodic mass-loss events of the evolved postAGB star in the Red Rectangle Nebula.

The most recent study shows low-resolution spectra in the UV, VIS and near-IR, using the DIS spectrograph (Dual Imaging Spectrograph) in the UV/VIS, and the TripleSpec near-IR spectrograph mounted at the $3.5 \mathrm{~m}$ telescope at the Apache Point Observatory (Wehres et al. 2014). In addition to a rich emission line spectrum in the near-IR, this study reveals a plethora of emission lines also in the UV and VIS.

Here, we present a follow-up study that employs mediumresolution échelle spectroscopy of the Red Square Nebula, using the X-shooter spectrograph, mounted on the VLT, Paranal, Chile. In Sect. 2 we give details on the observations, followed by Sect. 3 in which we present the full UV, VIS and near-IR spectra and their analysis, including a linelist with the identified spectral features. Section 4 compares the Red Square Nebula morphologically and chemically to the Red Rectangle Nebula. In Sect. 5 we conclude with the implications on the evolution of the Red Square Nebula.

\section{Observations}

Observations of the Red Square were obtained in summer 2010 and 2012 using the X-shooter spectrograph mounted on the Very Large Telescope (VLT), at the European Southern Observatory (ESO) in Paranal, Chile. The échelle spectrograph consists of three spectrometric arms; the UVB, covering the wavelength range between 300 and $550 \mathrm{~nm}$; VIS, covering the wavelength range between $550-1000 \mathrm{~nm}$, and near-IR, covering the wavelength range between 1000-2500 $\mathrm{nm}$. We observed the object with slit-dimensions of $11^{\prime \prime} \times 0.5^{\prime \prime}(\mathrm{UVB})$ and $11^{\prime \prime} \times 0.4^{\prime \prime}$ (VIS and near-IR), which amounts to the maximum spectral resolution of $R=\frac{\lambda}{\Delta \lambda}=9900,18200$ and 10500 for the UVB, VIS and near-IR arms, respectively.

Three different slit positions were used, depicted in Fig. 1. First, the slit was oriented along the three major symmetry axes of the nebula, with positions one and two along the walls of the outflow cones in summer 2012 (see also labels in Fig. 1), and one position in summer 2010, in which the slit was aligned perpendicular to the circumstellar disk, along the central part of the outflows. The parallactic angle of the Red Square Nebula is $\mathrm{PA} \simeq 46^{\circ}$, with an opening angle of the outflow cones of $\sim 106^{\circ}$. Hence, the position in 2010 is at PA $=46^{\circ}$, and the positions along the wall of the outflow cones were detained through VLT/NACO imaging of the Red Square Nebula which showed the limb brightened walls of the ouflow (Lenzen et al. 2003; Rousset et al. 2003). This lead to position angles for the 2012 observations of $\mathrm{PA}=6.5^{\circ}$ for position 1 , and $\mathrm{PA}=-97^{\circ}$ for position 2 .

Data reduction has been done using the ESO-X-shooter pipeline (version 2.2.0). For the 2010 observations, the UVB and VIS were obtained in staring mode, whereas in the nearIR the sky was subtracted by offsetting the telescope to a dark position well outside the nebula. No telluric standard star was observed in this period. For the 2012 observations, the sky was subtracted using a dedicated offset for all three arms of the spectrograph. Flux calibration for the UVB arm was done using the flux standard star GD153 taken in the beginning of the night with a wide slit $\left(5^{\prime \prime}\right)$ to minimize slit losses. For the VIS and nearIR arms, we used the B9V star HD 134218 both as a telluric and as a flux standard star. Telluric corrections in the VIS and

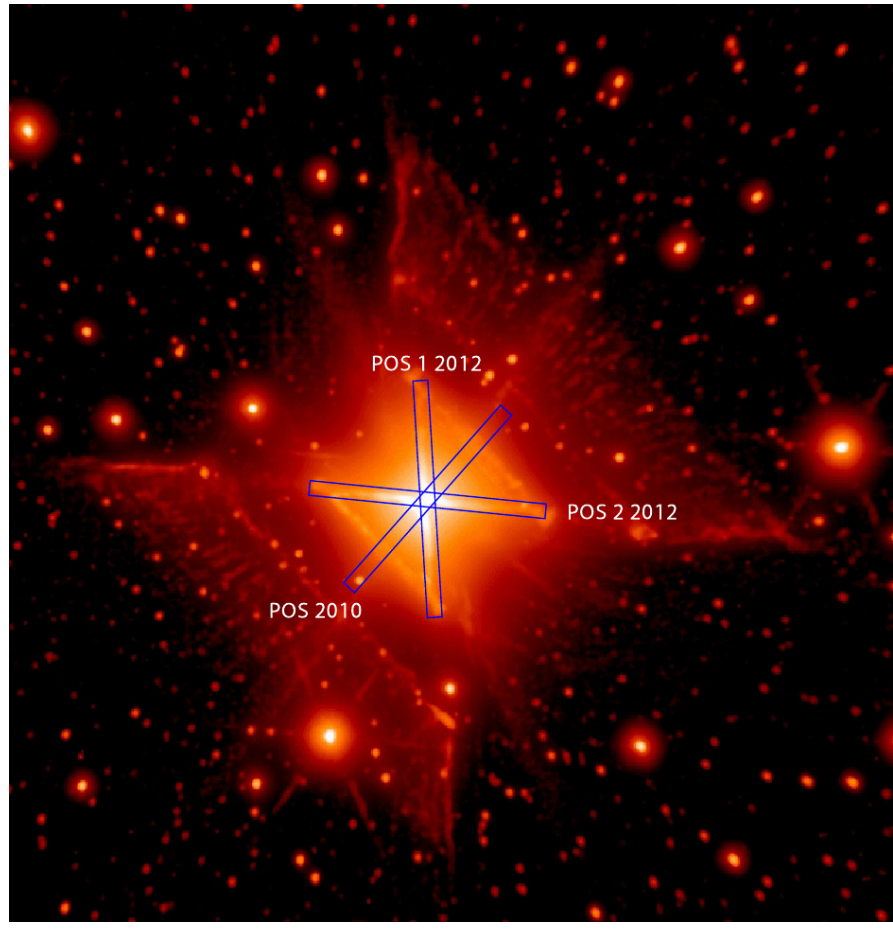

Fig. 1. Three slit positions for the observations are shown. The slit is oriented along the three symmetry axis of the Red Square Nebula, that is along the outflow walls and perpendicular to the circumstellar disk. North is up and east is to the left. (Credit: the $H$-band image was taken by Tuthill \& Lloyd 2007.)

Table 1. Observations used in this study.

\begin{tabular}{l|c|c|c|c}
\hline \hline Arm & Slit (") & $\Delta \lambda / \lambda$ & $\operatorname{PA}\left({ }^{\circ}\right)$ & $t_{\text {exp }}(\mathrm{s})$ \\
\hline \multicolumn{5}{c}{2010} \\
\hline UVB & $0.5 \times 11$ & 9900 & -46 & 2400 \\
VIS & $0.4 \times 11$ & 18000 & -46 & 2000 \\
near-IR & $0.4 \times 11$ & 10500 & -46 & 2000 \\
\hline \multicolumn{5}{|c|}{2012} \\
UVB & $0.5 \times 11$ & 9900 & 6.5 & 1200 \\
VIS & $0.4 \times 11$ & 18000 & 6.5 & 1340 \\
near-IR & $0.4 \times 11$ & 10500 & 6.5 & 600 \\
\hline UVB & $0.5 \times 11$ & 9900 & -97 & 1200 \\
VIS & $0.4 \times 11$ & 18000 & -97 & 1340 \\
near-IR & $0.4 \times 11$ & 10500 & -97 & 1200 \\
\hline
\end{tabular}

near-IR were performed using Spextool (Cushing et al. 2004; Vacca et al. 2003), using the well-known $B$ and $V$ magnitudes of this star to put the flux level of the spectrum on an absolute scale. In this case, a slit loss correction is not needed as the telluric star was observed using the same slit settings as the science target. For better overview we provide Table 1 with the observational details $^{1}$.

\section{Results and discussion}

The full spectrum for slit position 2 (2012) is shown in Fig. 2. We carefully checked the other two slit spectra (2010 and 2012, position 1) for significant changes compared to the 2012 (position 2)

1 The data-reduced spectra will be made publicly available through the Strasbourg astronomical Data Center (CDS) website. 


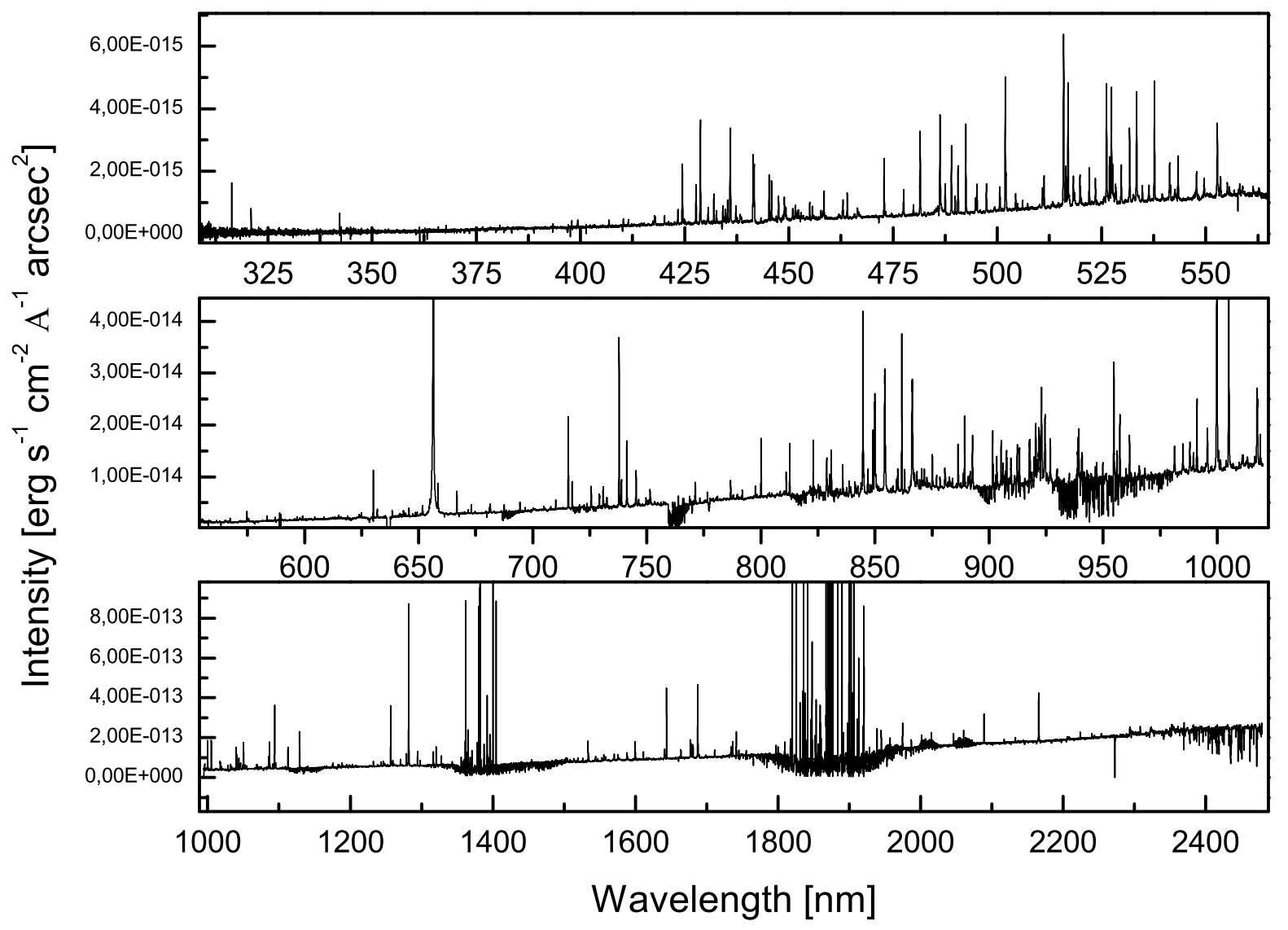

Fig. 2. X-shooter spectrum, obtained in 2012, using slit position 2. The spectra are the sum of all pixels of the slit. The three panels reflect the three spectrograph arms for the UVB (upper panel), VIS (middle panel) and near-IR (lower panel).

spectrum, that is changes in detected atomic species, strong fluctuations in intensity and/or a change in the line profiles. This also included a careful inspection of the individual pixel spectra for a given slit spectrum. This will be described in more detail in the following sections. Thus, whenever a specific emission line is discussed in the text, we will show the different slit spectra, decomposed to the individual pixel spectra for comparison, as this is crucial for some of the conclusions that are drawn. These plots also include a comparison spectrum that shows the residuals of two individual pixel spectra. If not otherwise mentioned in the text, the residuals will be from the spectrum taken in 2012 (position 2) at offsets of roughly +0.6" (pixel 30) and -2.5" (pixel 15) distance from the central star. Here, the star is located at the center of the slit. Dependent on the spectrum the center of the slit is located between pixel position 30-35. Negative distances to the central star thus belong to positions in the south-(east) of the slit (pixel values e.g., $\leq 30$ ), and positive distances belong to distances in the north-(west) of the star (i.e., pixel values $\geq 30$ ). Figures A.1-A.3 show the full spectral range in more detail, as well as a table with the identified emission lines (Table A.1). For emission line identifications the NIST database (Kramida et al. 2013) and "The Atomic Linelist v.2.04" (van Hoof 2014) are used. Diffuse interstellar absorption bands (DIBs) are also detected in the spectrum (Hobbs et al. 2009; Geballe et al. 2011; Cox et al. 2014).

Overall, the spectrum is characterized by strong permitted and forbidden emission lines, where the majority of the lines is due to atomic hydrogen and singly ionized Fe II and [Fe II] lines. In the whole spectral range we found emission bands of only one molecular species: $\mathrm{CO}$.
In addition Table A.1 categorizes the spectral features into (single peaked) emission line, double (peaked) emission line, absorption and P Cygni lines, as well as DIBs in the line of sight towards the Red Square Nebula. For every line this labelling is mentioned in Table A.1. Following this, we distinguish the following environments where the emission lines arise from:

1. Ca II showing a double peaked profile, indicative of a rotating disk (Aret et al. 2012; Aret \& Kraus 2015);

2. emission lines with P Cygni profiles indicating fast winds in the outflows (Israelian \& de Groot 1999);

3. H I recombination lines tracing the extended nebula and the disk photosphere;

4. forbidden emission lines, such as [N II] and [S II], trace low density, but fairly hot nebular gas;

5. permitted emission lines originate from the photosphere of the disk;

6. we place $[\mathrm{OI}]$ in the disk environment, separating the ionized photosphere from the neutral molecular gas, for example CO (Aret et al. 2012; Aret \& Kraus 2015);

7. diffuse interstellar absorption bands, indicating interstellar matter in the line of sight.

Furthermore, some of the emission lines show a broad underlying emission component, superimposed on a narrow emission profile, for instance [Fe II] emission lines. The narrow emission component is likely to originate from the photosphere of the disk, whereas the broad underlying component is indicative of shocks in the outflows. The next sections will discuss the identified species and the physical characteristics of their environments in more detail. 
Table 2. Line parameters for extinction correction using [Fe II] lines.

\begin{tabular}{c|c|c|c|c|c|c|c}
\hline \hline $\begin{array}{c}\lambda \\
\mathrm{nm}\end{array}$ & $\begin{array}{c}\text { Intensity } \\
\mathrm{erg} \mathrm{s}^{-1} \mathrm{~cm}^{-2} \mathrm{arcsec}^{-2}\end{array}$ & $\begin{array}{c}A \\
\mathrm{~s}^{-1}\end{array}$ & $J_{\text {up }}$ & $\lambda(1) / \lambda(2)$ & $\Delta \tau$ & $\begin{array}{c}\text { H I } \\
\mathrm{cm}^{-2}\end{array}$ & $A(V)$ \\
\hline \multicolumn{7}{c}{$\mathrm{b}^{4} F$} \\
\hline 441.68 & $4.39 \mathrm{E}-15 \pm 4 \mathrm{E}-16$ & $4.60 \mathrm{E}-01$ & $9 / 2$ & $680.98 / 441.68$ & 4.46 & $1.59 \mathrm{E} 22$ & $8.47 \pm 0.8$ \\
494.79 & $1.73 \mathrm{E}-15 \pm 2 \mathrm{E}-16$ & $5.90 \mathrm{E}-02$ & $9 / 2$ & $494.79 / 441.68$ & 1.24 & $1.51 \mathrm{E} 22$ & $8.05 \pm 0.8$ \\
680.98 & $1.34 \mathrm{E}-14 \pm 1 \mathrm{E}-15$ & $2.50 \mathrm{E}-02$ & $9 / 2$ & $680.98 / 494.79$ & 3.22 & $1.62 \mathrm{E} 22$ & $8.64 \pm 0.9$ \\
\hline 452.87 & $4.86 \mathrm{E}-16 \pm 5 \mathrm{E}-17$ & $3.98 \mathrm{E}-02$ & $5 / 2$ & $497.38 / 452.87$ & 0.84 & $1.24 \mathrm{E} 22$ & $6.62 \pm 0.7$ \\
497.38 & $3.59 \mathrm{E}-15 \pm 4 \mathrm{E}-16$ & $1.40 \mathrm{E}-01$ & $5 / 2$ & & \\
\hline \multicolumn{7}{c}{$\mathrm{c}^{2} G$} \\
\hline 567.36 & $4.42 \mathrm{E}-15 \pm 4 \mathrm{E}-16$ & $3.00 \mathrm{E}-01$ & $9 / 2$ & $1625.22 / 567.36$ & 5.10 & $1.37 \mathrm{E} 22$ & $7.27 \pm 0.7$ \\
1625.22 & $3.13 \mathrm{E}-14 \pm 3 \mathrm{E}-56$ & $3.70 \mathrm{E}-02$ & $9 / 2$ & & \\
\hline 583.59 & $4.34 \mathrm{E}-15 \pm 4 \mathrm{E}-16$ & $3.20 \mathrm{E}-01$ & $7 / 2$ & $1616.13 / 583.59$ & 5.04 & $1.42 \mathrm{E} 22$ & $7.58 \pm 0.8$ \\
848.06 & $2.21 \mathrm{E}-14 \pm 2 \mathrm{E}-15$ & $1.00 \mathrm{E}-01$ & $7 / 2$ & $848.06 / 583.59$ & 3.17 & $1.67 \mathrm{E} 22$ & $8.89 \pm 0.9$ \\
1616.13 & $5.50 \mathrm{E}-14 \pm 6 \mathrm{E}-15$ & $7.30 \mathrm{E}-02$ & $7 / 2$ & $1616.13 / 848.06$ & 1.87 & $1.13 \mathrm{E} 22$ & $6.03 \pm 0.6$ \\
\hline
\end{tabular}

Notes. The table consists of two parts: the first four columns are the line intensities measured in the spectra. The last 4 columns are the color excesses derived from the different line pairs. The extinction correction takes transitions from two different electronic states into account, that span $1.2 \mu \mathrm{m}$ in total.

\subsection{Atomic emission features}

\subsubsection{Extinction correction}

A plethora of forbidden and permitted Fe II lines can be identified. Here, two sets of transitions with the same upper level provide a measure of the extinction (color excess). We have put these on an absolute optical depth scale by using the relation between color excess and total visual extinction provided from Draine (2003) or equivalently, an equivalent total H column density using $N_{\mathrm{H}}=1.9 \times 10^{-21} A(V)$. The emission lines that we used for the extinction correction are given in Table 2. We used two different electronic states of $[\mathrm{Fe} I \mathrm{II}]$, i.e., $\mathrm{b}^{4} \mathrm{~F}$ and $\mathrm{c}^{2} \mathrm{G}$. The transitions span all three parts of the échelle gratings (UV, VIS and near-IR). $A(V)$ is then given with an average value of 7.69 when all values are considered. The errors on the final $A(V)$ depend here on the uncertainties in the intensities of the emission lines, which is estimated to be in the order of $10 \%$. An additional error is also introduced when using Draine (2003) in which the uncertainty in wavelength also has an influence on the H-atom column density, and thus on $A(V)$. Here, we use the relationship for $A_{\lambda} / A(V)$ provided by Cardelli et al. (1989) and using an $R_{V}=3.1$ (for the general ISM). We note that the DIB strength in the Red Square seems to be comparable to the DIB strength seen towards BD+63 1964, which has an $A(V) \sim 3.1$ mag (Ehrenfreund et al. 1997; Tuairisg et al. 2000). This would suggest that half of the measured extinction is due to interstellar extinction and about half of it is due to circumstellar extinction.

\subsubsection{The excitation temperature of [Fe II]}

An overview of all detected energy levels of [Fe II] and Fe II can be seen in Table A.1. The identifications are based on NIST (Kramida et al. 2013) and "The Atomic Linelist v.2.04" (van Hoof 2014). Transitions that were lacking Einstein $A$ coefficients $\left(\mathrm{s}^{-1}\right)$ in the NIST atomic database - mainly for Fe II and [Fe II] transitions - were adopted from Garstang (1962) or Nussbaumer \& Storey (1980, 1988).

Most of the observed transitions come from energy levels with excitation energies less than $40000 \mathrm{~cm}^{-1}$. Only a small fraction of energy transitions come from energy levels higher than 40000 . This multitude of linked energy levels provides us with a very sensitive tool to allow an estimate on the temperature.

Therefore, we took a total of 40 transitions into account, spanning a range of almost $20000 \mathrm{~cm}^{-1}$ and 6 different multiplets of $[\mathrm{Fe} \mathrm{II}]$ in order to calculate excitation temperatures. The result is plotted in Fig. 3. The individual transitions that have been taken into account are given in Table 3. From the Boltzmann diagram we arrive at an excitation temperature of around $6700 \mathrm{~K}$.

In addition Pesenti et al. (2003) have designed two diagnostic diagrams that enable us to derive density and temperature estimates of the emitting gas from near-IR line ratios. Specifically, the [Fe II] $0.8617 \mu \mathrm{m} /[\mathrm{Fe}$ II] $1.257 \mu \mathrm{m}$ and [Fe II] $1.533 \mu \mathrm{m} /[\mathrm{Fe}$ II] $1.644 \mu \mathrm{m}$ ratios imply densities of $5 \times 10^{4}-1 \times 10^{5} \mathrm{~cm}^{-3}$ and temperatures of 7000-10000 K. This is confirmed by the [Fe II] $1.257 \mu \mathrm{m} /$ [S II] $1.03 \mu \mathrm{m}$ and [Fe II] $1.533 \mu \mathrm{m} /[\mathrm{Fe} \mathrm{II}] 1.644 \mu \mathrm{m}$ line ratios which also result in $5 \times 10^{4}-1 \times 10^{5} \mathrm{~cm}^{-3}$ and $7000-10000 \mathrm{~K}$ gas temperature.

We compared our results to the results by Rudy et al. (1992). In their study the authors concluded on $T_{\mathrm{e}} \sim 5000 \mathrm{~K}$ and $N_{\mathrm{e}}=1 \times 10^{5} \mathrm{~cm}^{-3}$ in their near-IR spectrum. This temperature is lower than the excitation temperature found in our study. This might be due to the fact that their upper state energies come from a narrow range of upper state energies. All of the [Fe II] lines come from the same upper level $\left(\mathrm{a}^{4} \mathrm{D}\right)$, taking into account 7 transitions, whereas our study spans a range of almost $20000 \mathrm{~cm}^{-1}$ in upper state energies, with a total of 6 multiplets including 40 transitions.

In conclusion, we derive an electron temperature of at least $7000 \mathrm{~K}$ and an electron density of $8 \times 10^{4} \mathrm{~cm}^{-3}$ for the [Fe II] emitting gas.

\subsubsection{The Call triplet - a double peaked profile}

Figure 4 shows the permitted Ca II triplet at $\lambda=849 \mathrm{~nm}$, $\lambda=854 \mathrm{~nm}$, and $\lambda=866 \mathrm{~nm}$, respectively. The $x$-axis is corrected for the local standard of rest of $12.12 \mathrm{~km} \mathrm{~s}^{-1}$. Zero $\mathrm{km} \mathrm{s}^{-1}$ in the figure corresponds to the wavelength given from the NIST database $\left(\lambda_{\text {air }}\right)$ (Kramida et al. 2013). The intensity ratio of the lines is about $1: 1: 1$. The Einstein $A$ coefficients for all three 


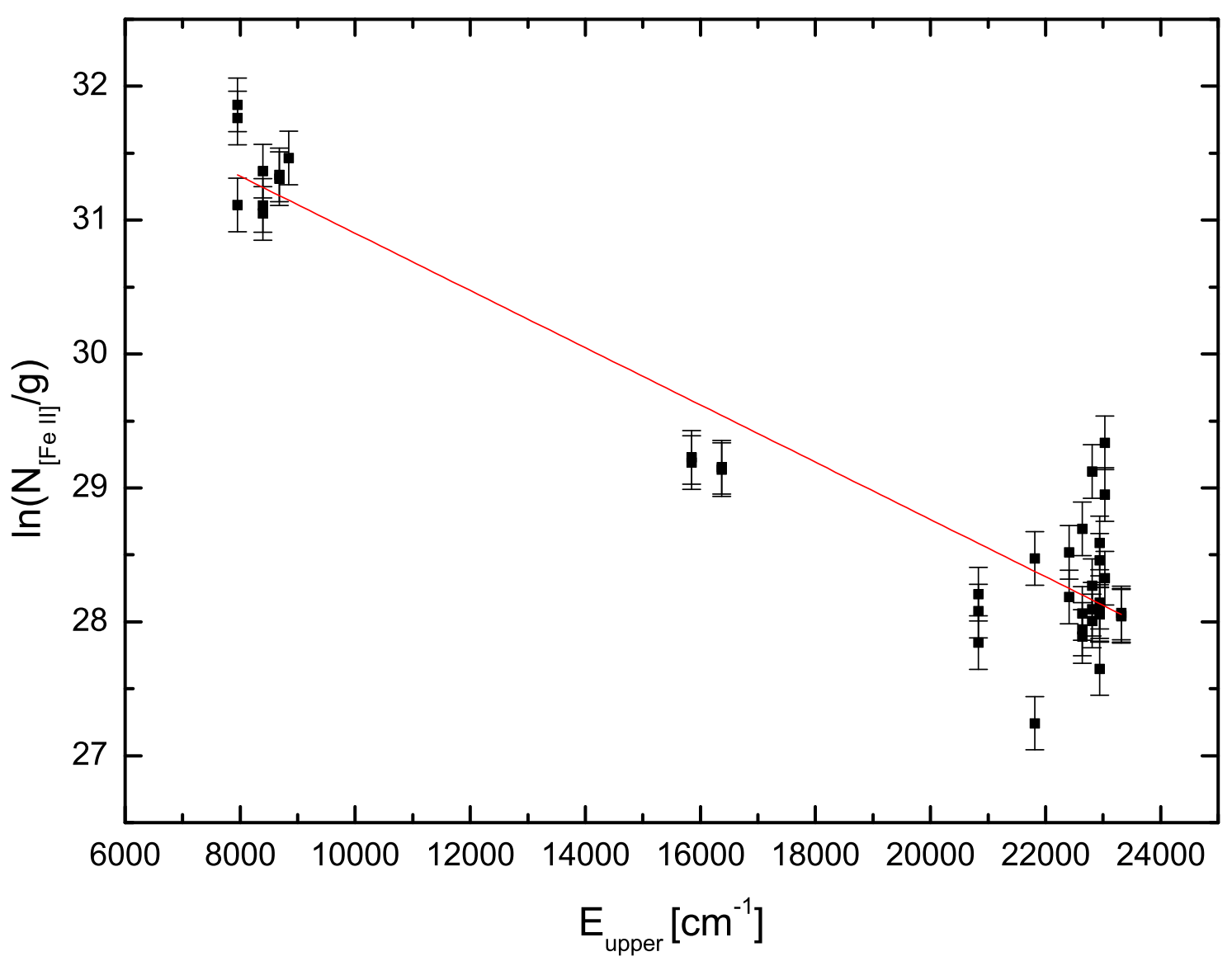

Fig. 3. Boltzmann excitation diagram for a selection of 40 [Fe II] emission lines with $N$ and $g$ the column density and statistical weight of the upper level. The slope indicates an excitation temperature of $6700 \mathrm{~K}$.

emission lines are: $A=1.11 \times 10^{6} \mathrm{~s}^{-1}$ at $\lambda=849 \mathrm{~nm}, A=9.9 \times$ $10^{6} \mathrm{~s}^{-1}$ at $\lambda=854 \mathrm{~nm}$, and $A=1.06 \times 10^{7} \mathrm{~s}^{-1}$ at $\lambda=866 \mathrm{~nm}$. In case of an optically thin environment, we would expect an emission line ratio of 1:9:5 for the Ca II triplet. This has been detected for example in Herbig-Haro objects (Reipurth et al. 1986). In contrast, an intensity ratio of 1:1:1 implies saturation from an optically thick environment, as argued by Reipurth et al. (1986), and might be indicative for an accretion-related origin. This suggestion is strengthened by a study by Rudy et al. (1992), who argues that Ca II comes from a dense $\left(N_{\mathrm{e}}=10^{10} \mathrm{~cm}^{-3}\right)$, warm (4000-8000 K) environment in MWC 922. Rudy et al. (1992) argued that the observed intensity ratios are also indicative of an optical thick environment with a minimum optical depth of 30 at $854 \mathrm{~nm}$. This in turn was used to imply a minimum column density of hydrogen of $2 \times 10^{19} \mathrm{~cm}^{-2}$. If their detected lines were broadened by mass motions of $20 \mathrm{~km} \mathrm{~s}^{-1}$, this would imply a column density of an order of magnitude higher.

We can furthermore strengthen the argument that Ca II exists in the disk environment by the fact that the Ca II triplet shows a clear double peaked profile resolved with the X-shooter spectrograph and shown in Fig. 4. From this figure the FWHM of the emission peaks can be estimated to be around $150 \mathrm{~km} \mathrm{~s}^{-1}$. We also note an emission plateau underlying all three Ca II lines. This broad plateau shows velocities as high as $100 \mathrm{~km} \mathrm{~s}^{-1}$ on the red shifted side, whereas the blue shifted side extends to about $200 \mathrm{~km} \mathrm{~s}^{-1}$. The velocity separation between the two maxima is around $50 \mathrm{~km} \mathrm{~s}^{-1}$. That means that the gas would be rotating with a projected velocity of around $25 \mathrm{~km} \mathrm{~s}^{-1}$. We also compared the 2012 (position 2) spectrum to the 2010 spectrum. In both spectra we took the sum of a couple of pixels at the center (around the central star) and edge of the slit, and compared the Ca II emission bands. If we assume the origin of those $\mathrm{Ca}$ II bands in a central region in the disk, then the double peaked profile should disappear as we move outward from the star (center of the slit). This is not the case. The double peaked profile is clearly visible at both slit positions (2010 and 2012, position 2), and it also makes no difference whether we look at the pixels in the center of the slit or at the edge. For a better comparison we also show the spectra taken in 2010 and 2012 (position 2) decomposed into the individual pixel spectra in Fig. 5. The pixel number is given, as well as the distance from the central star. It can clearly be seen that the 2012 (position 2) and 2010 pixel positions resemble each other with respect to the content of atomic species and line profiles, as well as intensity ratios between the detected species. Apart from a decrease in intensity with distance to the central star, there is barely any spectral change for the Ca II double peaks. For a more quantitative assessment, we also plot the residuals of two individual pixel spectra of 2012 (position 2). The residuals are plotted in the bottom-most spectrum. Here, we choose to subtract one spectrum which is close to the star (at $+0.6^{\prime \prime}$ distance, i.e., pixel 30) from one spectrum which is at roughly $-2.5^{\prime \prime}$ (pixel 15) distance to the central star. Here, intensity fluctuations in the Ca II species are in the order of $10 \%$.

At first sight the detection of Ca II along the full length of the slit spectrum seems to contradict our conclusion that Ca II is located in the disk only. However, following the argumentation for the Red Rectangle Nebula of Cohen et al. (2004), and given 
A\&A 601, A69 (2017)

Table 3. Parameters for determining the excitation temperature and column density of [Fe II].

\begin{tabular}{|c|c|c|c|c|c|}
\hline $\begin{array}{c}\text { Transition } \\
\mathrm{nm}\end{array}$ & $\begin{array}{c}\text { Area } \\
\operatorname{erg~s}^{-1} \mathrm{~cm}^{-2} \operatorname{arcsec}^{-2}\end{array}$ & $\begin{array}{c}\text { Einstein } A \\
\mathrm{~s}^{-1}\end{array}$ & $\begin{array}{c}E_{\text {up }} \\
\mathrm{cm}^{-1}\end{array}$ & $g_{\text {up }}$ & $\ln [N / g]$ \\
\hline \multicolumn{6}{|c|}{$a^{6} D-a^{6} s$} \\
\hline 445.25 & $3.51 \mathrm{E}-11 \pm 4 \mathrm{E}-12$ & $4.65 \mathrm{E}-1$ & 23317.64 & 6 & $28.04 \pm 0.2$ \\
\hline 441.41 & $5.60 \mathrm{E}-11 \pm 6 \mathrm{E}-12$ & $7.30 \mathrm{E}-1$ & 23317.64 & 6 & $28.05 \pm 0.2$ \\
\hline 447.53 & $1.75 \mathrm{E}-11 \pm 2 \mathrm{E}-12$ & $2.27 \mathrm{E}-1$ & 23317.64 & 6 & $28.07 \pm 0.2$ \\
\hline \multicolumn{6}{|c|}{$a^{6} D-b^{4} F$} \\
\hline 441.66 & $5.43 \mathrm{E}-11 \pm 5 \mathrm{E}-12$ & $4.19 \mathrm{E}-1$ & 22637.20 & 10 & $28.06 \pm 0.2$ \\
\hline 443.28 & $2.50 \mathrm{E}-12 \pm 3 \mathrm{E}-13$ & $4.88 \mathrm{E}-2$ & 22939.35 & 6 & $27.65 \pm 0.2$ \\
\hline 445.83 & $3.21 \mathrm{E}-11 \pm 3 \mathrm{E}-12$ & $2.55 \mathrm{E}-1$ & 22810.35 & 8 & $28.27 \pm 0.2$ \\
\hline 448.92 & $1.75 \mathrm{E}-11 \pm 2 \mathrm{E}-12$ & $1.35 \mathrm{E}-1$ & 22939.35 & 6 & $28.59 \pm 0.2$ \\
\hline 449.28 & $1.34 \mathrm{E}-11 \pm 1 \mathrm{E}-12$ & $5.61 \mathrm{E}-2$ & 22637.20 & 10 & $28.69 \pm 0.2$ \\
\hline 450.97 & $9.44 \mathrm{E}-12 \pm 9 \mathrm{E}-13$ & $5.2 \mathrm{E}-2$ & 23031.28 & 4 & $29.34 \pm 0.2$ \\
\hline 451.56 & $1.31 \mathrm{E}-11 \pm 1 \mathrm{E}-12$ & $6.0 \mathrm{E}-2$ & 22810.35 & 6 & $29.12 \pm 0.2$ \\
\hline 452.87 & $4.48 \mathrm{E}-12 \pm 4 \mathrm{E}-13$ & $3.98 \mathrm{E}-2$ & 22939.35 & 6 & $28.46 \pm 0.2$ \\
\hline 453.35 & $1.78 \mathrm{E}-12 \pm 2 \mathrm{E}-13$ & $1.45 \mathrm{E}-2$ & 23031.28 & 4 & $28.95 \pm 0.2$ \\
\hline \multicolumn{6}{|c|}{$\mathrm{a}^{6} D-\mathrm{b}^{4} \mathrm{P}$} \\
\hline 464.00 & $1.33 \mathrm{E}-11 \pm 1 \mathrm{E}-12$ & $4.67 \mathrm{E}-1$ & 20830.55 & 2 & $28.21 \pm 0.2$ \\
\hline 466.47 & $5.70 \mathrm{E}-12 \pm 6 \mathrm{E}-13$ & $1.47 \mathrm{E}-1$ & 22409.82 & 2 & $28.52 \pm 0.2$ \\
\hline 472.88 & $2.48 \mathrm{E}-11 \pm 2 \mathrm{E}-12$ & $4.53 \mathrm{E}-1$ & 22409.82 & 4 & $28.18 \pm 0.2$ \\
\hline 477.24 & $4.86 \mathrm{E}-13 \pm 5 \mathrm{E}-14$ & $2.3 \mathrm{E}-2$ & 21812.05 & 4 & $27.84 \pm 0.2$ \\
\hline 479.86 & $4.86 \mathrm{E}-12 \pm 5 \mathrm{E}-13$ & $6.75 \mathrm{E}-2$ & 21812.05 & 4 & $28.47 \pm 0.2$ \\
\hline 489.00 & $2.43 \mathrm{E}-11 \pm 2 \mathrm{E}-12$ & $3.41 \mathrm{E}-1$ & 20830.55 & 6 & $28.08 \pm 0.2$ \\
\hline 495.88 & $2.78 \mathrm{E}-13 \pm 3 \mathrm{E}-14$ & $5.0 \mathrm{E}-3$ & 20830.55 & 6 & $27.84 \pm 0.2$ \\
\hline \multicolumn{6}{|c|}{$a^{4} F-b^{4} F$} \\
\hline 494.79 & $5.74 \mathrm{E}-12 \pm 6 \mathrm{E}-13$ & $5.90 \mathrm{E}-2$ & 22637.21 & 10 & $27.89 \pm 0.2$ \\
\hline 495.11 & $1.02 \mathrm{E}-11 \pm 1 \mathrm{E}-12$ & $1.70 \mathrm{E}-1$ & 23031.30 & 4 & $28.33 \pm 0.2$ \\
\hline 497.37 & $9.81 \mathrm{E}-12 \pm 1 \mathrm{E}-12$ & $1.4 \mathrm{E}-1$ & 22939.36 & 6 & $28.08 \pm 0.2$ \\
\hline 504.39 & $4.39 \mathrm{E}-12 \pm 4 \mathrm{E}-13$ & $6.50 \mathrm{E}-2$ & 22939.36 & 6 & $28.05 \pm 0.2$ \\
\hline 477.51 & $1.28 \mathrm{E}-11 \pm 2 \mathrm{E}-12$ & $1.30 \mathrm{E}-1$ & 22810.36 & 8 & $28.09 \pm 0.2$ \\
\hline 481.48 & $4.22 \mathrm{E}-11 \pm 4 \mathrm{E}-12$ & $4.00 \mathrm{E}-1$ & 22637.21 & 10 & $27.94 \pm 0.2$ \\
\hline 487.48 & $1.30 \mathrm{E}-11 \pm 1 \mathrm{E}-12$ & $1.7 \mathrm{E}-1$ & 22939.36 & 6 & $28.14 \pm 0.2$ \\
\hline 490.57 & $1.94 \mathrm{E}-11 \pm 2 \mathrm{E}-12$ & $2.2 \mathrm{E}-1$ & 22810.36 & 8 & $28.01 \pm 0.2$ \\
\hline \multicolumn{6}{|c|}{$a^{6} D-a^{4} D$} \\
\hline 1248.63 & $2.56 \mathrm{E}-13 \pm 3 \mathrm{E}-14$ & $4.7 \mathrm{E}-4$ & 8391.4 & 6 & $31.05 \pm 0.2$ \\
\hline 1252.20 & $2.00 \mathrm{E}-11 \pm 2 \mathrm{E}-12$ & 7.3E-4 & 8846.77 & 2 & $31.46 \pm 0.2$ \\
\hline 1256.76 & $7.70 \mathrm{E}-12 \pm 8 \mathrm{E}-13$ & $4.74 \mathrm{E}-3$ & 7955.30 & 8 & $31.86 \pm 0.2$ \\
\hline 1270.44 & $7.42 \mathrm{E}-13 \pm 7 \mathrm{E}-14$ & $3.9 \mathrm{E}-3$ & 7955.30 & 2 & $31.11 \pm 0.2$ \\
\hline 1278.87 & $1.37 \mathrm{E}-12 \pm 1 \mathrm{E}-13$ & $2.9 \mathrm{E}-3$ & 8680.45 & 4 & $31.34 \pm 0.2$ \\
\hline 1294.38 & $1.59 \mathrm{E}-12 \pm 2 \mathrm{E}-13$ & $2.2 \mathrm{E}-3$ & 8391.94 & 6 & $31.37 \pm 0.2$ \\
\hline 1297.86 & $5.90 \mathrm{E}-13 \pm 6 \mathrm{E}-14$ & $1.3 \mathrm{E}-3$ & 8680.45 & 4 & $31.31 \pm 0.2$ \\
\hline 1320.63 & $1.96 \mathrm{E}-12 \pm 2 \mathrm{E}-13$ & $1.40 \mathrm{E}-3$ & 7955.30 & 8 & $31.76 \pm 0.2$ \\
\hline 1327.86 & $7.61 \mathrm{E}-13 \pm 8 \mathrm{E}-14$ & $1.4 \mathrm{E}-3$ & 8391.94 & 6 & $31.11 \pm 0.2$ \\
\hline \multicolumn{6}{|c|}{$a^{4} F-a^{2} G$} \\
\hline 715.58 & $3.74 \mathrm{E}-11 \pm 4 \mathrm{E}-12$ & $1.46 \mathrm{E}-1$ & 15844.65 & 10 & $29.23 \pm 0.2$ \\
\hline 717.26 & $1.03 \mathrm{E}-11 \pm 1 \mathrm{E}-12$ & $5.51 \mathrm{E}-2$ & 16369.36 & 8 & $29.14 \pm 0.2$ \\
\hline 738.88 & $7.76 \mathrm{E}-12 \pm 8 \mathrm{E}-13$ & $4.21 \mathrm{E}-2$ & 16369.36 & 8 & $29.15 \pm 0.2$ \\
\hline 745.32 & $1.13 \mathrm{E}-11 \pm 1 \mathrm{E}-12$ & 4.77E-2 & 15844.65 & 10 & $29.19 \pm 0.2$ \\
\hline
\end{tabular}




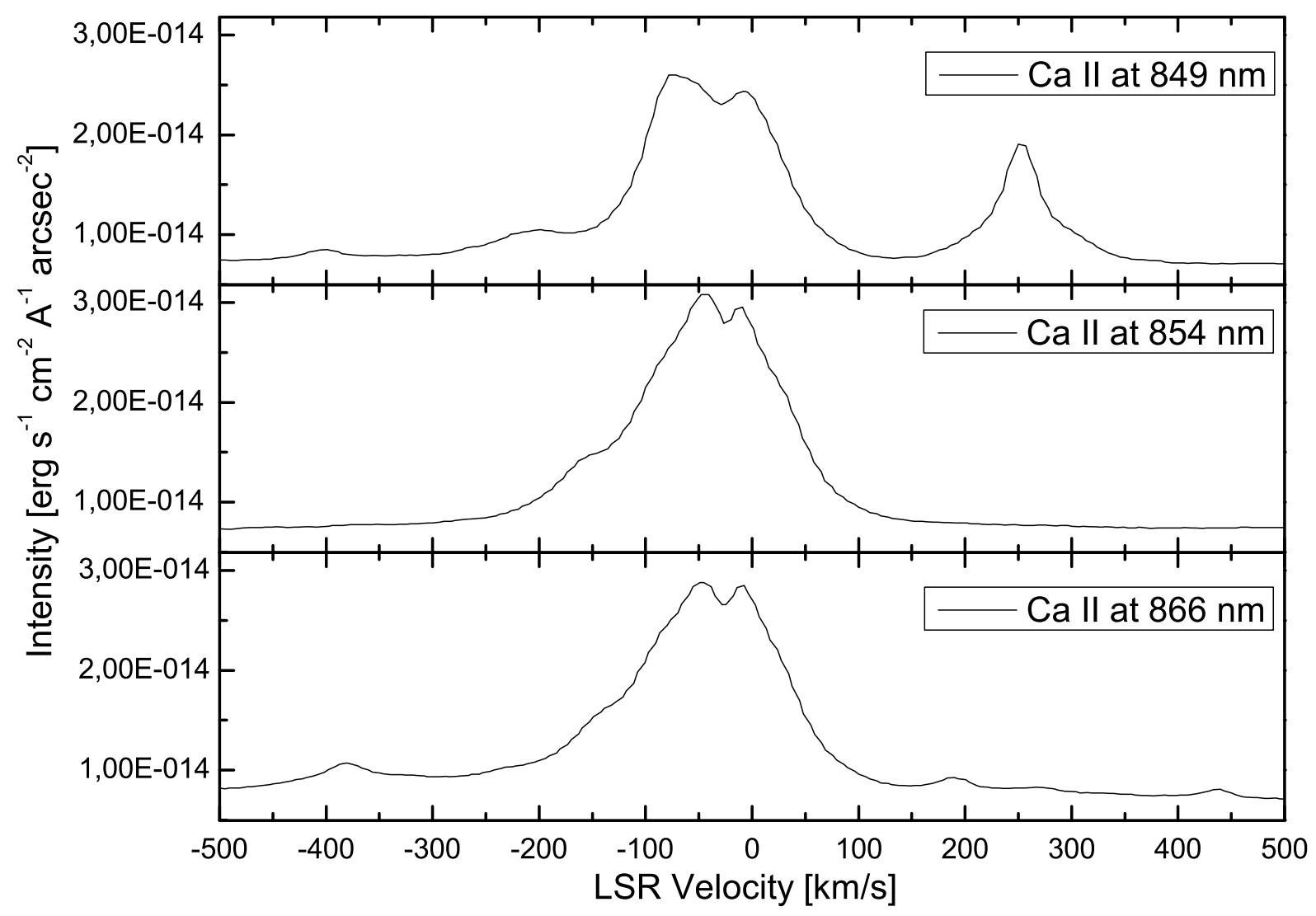

Fig. 4. Ca II triplet emission lines. The wavelength is corrected for the local standard of rest (LSR $=12.12 \mathrm{~km} \mathrm{~s}^{-1}$ ). The zero point is equal to the wavelength $\left(\lambda_{\text {air }}\right)$ given from the NIST database. The intensity ratios of the three lines are about 1:1:1, indicative of an optically thick environment. The double peaked profile is indicative that the lines originate in a circumstellar and rotating disk.

the geometry of the Red Square Nebula, the conclusion that Ca II is located in the disk seems inescapable. Here, we will argue that $\mathrm{Ca}$ II is indeed formed in the inner part of a rotating disk. The geometry of the Nebula is dominated by a cone structure similar as seen in the Red Rectangle Nebula for which a hollow bicone surface was invoked (Cohen et al. 2004). In our study, the slit spectra are oriented along the limb-brightened walls of the cone as well as along the central axis. Hence, all the light we see is scattered by dust in the inner rim of the bicone walls. As the scattering angle does not change, the spectral signature of the disk rotation will also not change, and hence no change in the line profiles of the individual pixel spectra is expected.

The [Ca II] and Ca II triplet lines at $730 \mathrm{~nm}$, and around $850 \mathrm{~nm}$, as well as the [O I] lines at 558, 630, and $636 \mathrm{~nm}$ and the $\mathrm{H} \alpha$ line have been used before as disk indicators for $\mathrm{B}[\mathrm{e}] \mathrm{su}-$ pergiant stars (Aret et al. 2012; Aret \& Kraus 2015). The kinematics of the disk was determined by comparing the line profiles of the different sets of emission lines. Following the approach by Aret et al. (2012), we do detect a double peak profile in the Ca II triplet. The [Ca II] lines around $730 \mathrm{~nm}$ are located in a region which is dominted by atmospheric absorption bands. We carefully looked at several individual pixel spectra which all show clear indication of the double peak profile and overplotted a simulated atmospheric transmission spectrum. This gave additional evidence that the double peak profile in the Ca II around $730 \mathrm{~nm}$ shows real signatures from the Red Square. Figure 6 shows again several individual pixel spectra. The residual spectrum of the spectra at $+0.6^{\prime \prime}$ and $-2.5^{\prime \prime}$ is plotted in the bottom-most panel. Here, the residuals are in the same order as the baseline noise. However, both, the morphology of the nebula, and the double peaked line profiles suggest that we are seeing a disk from the side.

\subsubsection{HI recombination lines - $P$ Cygni lines}

Figure 7 shows a selection of H I Balmer transitions between $397 \mathrm{~nm}$ and $656 \mathrm{~nm}$ in the upper panel. Again, a velocity correction of LSR $=12.12 \mathrm{~km} \mathrm{~s}^{-1}$ for the local standard of rest is applied. The zero-point is set to the wavelength given in the NIST database $\left(\lambda_{\text {air }}\right)$ (Kramida et al. 2013). The transition process from H I absorption lines (H I 2-7) towards a P Cygni line profile starting with (H I 2-6) is shown. The P Cygni profiles are indicative of an extended shell of gas moving away from the central star (Israelian \& de Groot 1999). The H I lines show a $F W H M=100 \mathrm{~km} \mathrm{~s}^{-1}$ and are good tracers of the structure of the extended nebula. For most of the H I Balmer lines we do not detect a change in the line profiles with change in slit position. For a better comparison we plot the Red Square spectrum obtained in 2010 and the spectrum obtained in 2012, position 2, decomposed into the individual pixel spectra (see Fig. 8). The P Cygni line profile of the $\mathrm{H} \beta$ emission line is clearly visible for both slit positions and again only a decrease in intensity is detected with distance from the star. Again, we also plot the residual spectrum in the bottom-most panel. The residuals have been obtained by subtracting pixel spectrum $30\left(+0.6^{\prime \prime}\right)$ from pixel spectrum 15 $\left(-2.5^{\prime \prime}\right)$ of the slit spectrum taken in 2012, position 2. The residuals in Fig. 8 cannot be distinguished from the baseline noise. A zoom-in on the $\mathrm{H} \beta$ emission line is also shown in Fig. 9 for better comparison.

In addition, we show the individual pixel spectra for the $\mathrm{H} \alpha$ emission line in which also the P Cygni line profile can be 


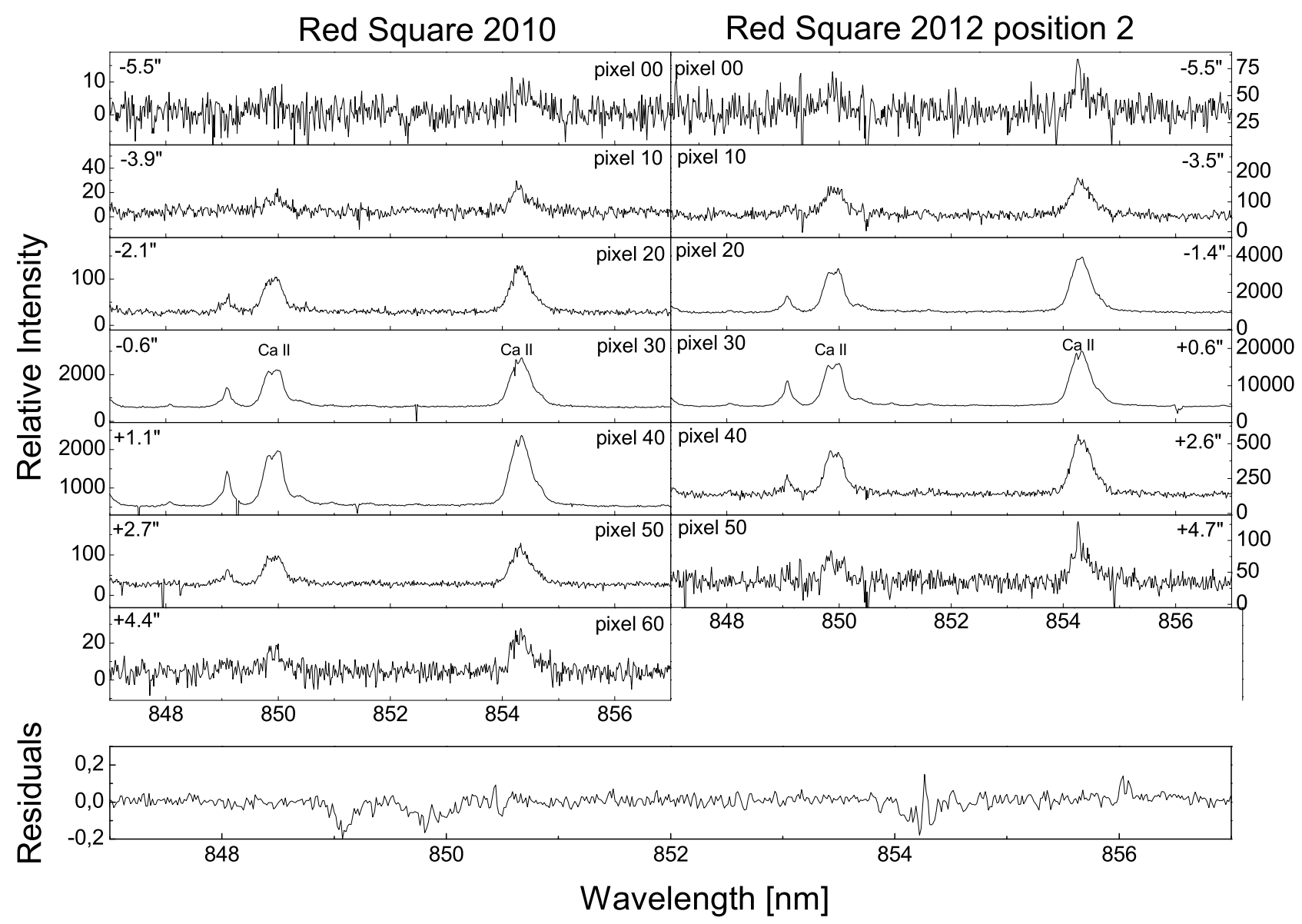

Fig. 5. Some of the individual pixel spectra of Ca II are shown. The left panel shows the spectra from 2010, and the right panel shows the spectra taken in 2012, position 2. Pixel 00 (2010) corresponds to the south-east position. Pixel 00 for position 2 in 2012 is located in the east. The intensities of the lines decrease with distance to the central star, but the double peaked line profiles as well as the atomic content of the spectra do not show any significant changes. The bottom-most panel gives the residuals of the slit spectrum in 2012 (position 2) when the spectrum at $+0.6^{\prime \prime}$ (pixel 30) is subtracted from the spectrum at $-2.5^{\prime \prime}$ (pixel 15) distance to the central star. Negative distances indicate directions in the south-(east) of the nebula. Positive distances indicate directions towards the north-(west).

detected. We plot the spectra obtained in 2010 and 2012, as well as the individual decomposed pixel spectra (see Fig. 10). Especially for larger distances from the central star, we note that the P Cygni profile seems more pronounced in the 2010 dataset, compared to the dataset in 2012, position 2. This hints towards the fact that the outflows are centrally located on the (pole of the) star. This is conclusive when also looking at the residual spectrum in the bottom-most panel. Again, the spectrum tracing $+0,6^{\prime \prime}$ has been subtracted from the spectrum at $-2.5^{\prime \prime}$ distance to the central star. Here, the residuals are about twice as large (about 25\%) compared to the Ca II triplet lines.

\subsubsection{Dipole forbidden lines - low density gas tracers [S II] and [N II]}

Forbidden lines can be used as a probe for low density regions. The [S II] emission features at 406.9 and $407.6 \mathrm{~nm}$, as well as at $671.7,673.1$ and $1032.1 \mathrm{~nm}$ are often used as density tracers in circumstellar shells, as the first two lines at $400 \mathrm{~nm}$ appear in preference over the emission lines at 671 and $673 \mathrm{~nm}$ if the density in circumstellar shells is high (Allen \& Swings 1976). Here, we detect weak [S II] emission at $406.9 \mathrm{~nm}$. The emission line at $407.6 \mathrm{~nm}$ is completely absent. The absence of the $407.6 \mathrm{~nm}$ [S II] line in our spectrum supports the hypothesis of a low density origin. The [S II] emission lines at $\lambda=671.7,673.1 \mathrm{~nm}$ and at $1032.1 \mathrm{~nm}$ are clearly present here, and can be used as an estimate for the density (Hamann 1994). The first two [S II] transitions connect states of the same parity and are hence socalled dipole forbidden transitions: $\lambda \lambda 6717\left({ }^{4} \mathrm{~S}_{3 / 2}^{\mathrm{o}}-{ }^{2} \mathrm{D}_{5 / 2}^{\mathrm{o}}\right)$ and $\lambda \lambda 6731\left({ }^{4} \mathrm{~S}_{3 / 2}^{\mathrm{o}}-{ }^{2} \mathrm{D}_{3 / 2}^{\mathrm{o}}\right)$. These two lines can be excited by collisions, and since their upper state energies are about the same (14885 $\mathrm{cm}^{-1}$ and $14852 \mathrm{~cm}^{-1}$ for the $\Omega=5 / 2$ and $\Omega=3 / 2$, respectively), the de-excitation is solely governed by the ratio of the Einstein $A$ coefficients of $2.01 \times 10^{-4} \mathrm{~s}^{-1}$ and $5.75 \times 10^{-4} \mathrm{~s}^{-1}$ in the low density regime, where collisions hardly occur. Following Hamann (1994) we can determine the electron densities from the line ratios of the aformentioned transitions and we arrive at an electron density of $3 \times 10^{4} \mathrm{~cm}^{-3}$. This density is very similar to that derived for the $[\mathrm{Fe}$ II $]$ emitting gas (between $5 \times 10^{4}$ and $\left.1 \times 10^{5} \mathrm{~cm}^{-3}\right)$.

The [N II] emission lines at 654.8 and $658.4 \mathrm{~nm}$ are affected by the $\mathrm{H} \alpha$ emission line. Although present in our spectra, we did not attempt to use these lines for getting estimates on the electronic temperatures or densities. However, a zoom-in on the $\mathrm{H} \alpha$ line reveals that the low density gas tracers (see Fig. 11) are present in both slit positions, that is in 2010 and 2012, position 2. The emission lines are strongest in the center of the slit position and their intensities decrease with distance to the central star. The bottom-most spectrum shows the residuals by subtracting the individual pixel spectra at $+0.6^{\prime \prime}$ from the spectrum at 


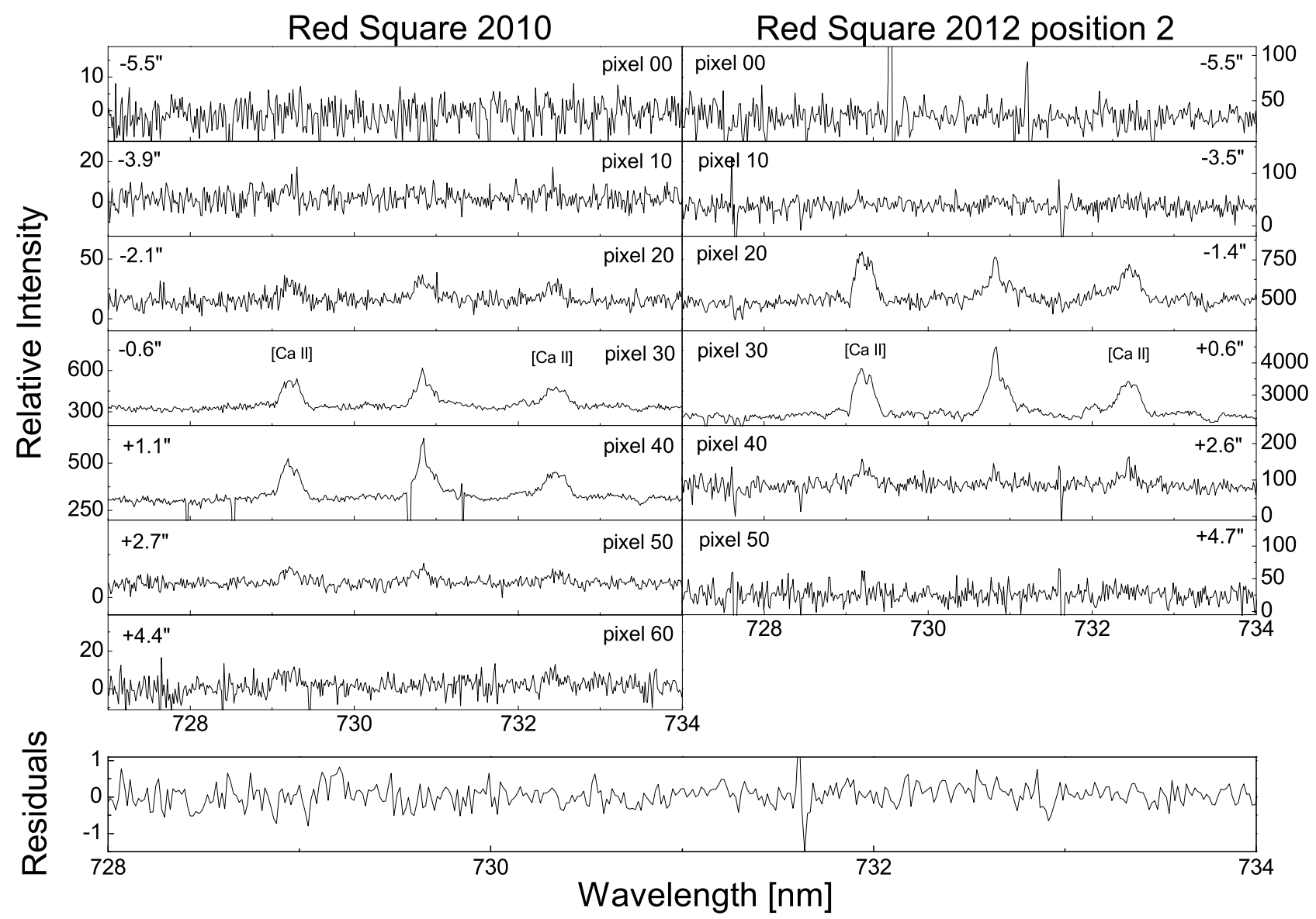

Fig. 6. Some of the individual pixel spectra of [Ca II] lines are shown. The left panel shows the spectra from 2010, and the right panel shows the spectra taken in 2012, position 2. Pixel 00 (2010) corresponds to the south-east position. Pixel 00 for position 2 in 2012 is located in the east. Since the $[\mathrm{Ca}$ II] lines are located in a region which is dominated by atmospheric absorption lines, it is hard to determine whether the substructure seen on the [Ca II] lines is intrinsic. The bottom-most panel gives the residuals of the slit spectrum in 2012 (position 2) when the spectrum at $+0.6^{\prime \prime}$ (pixel 30) is subtracted from the spectrum at $-2.5^{\prime \prime}$ (pixel 15) distance to the central star. Negative distances indicate directions in the south-(east) of the nebula. Positive distances indicate directions towards the north-(west).

$-2.5^{\prime \prime}$ in the 2012 (position 2) dataset. Residuals in the order of around $5 \%$ can be detected at $658.4 \mathrm{~nm}$. We furthermore notice an absorption band at pixel position 00, in the 2010 dataset. This absorption component changes to the emission line seen closer to the star (pixel 30, 2010). This absorption is statistically significant $(4 \sigma)$, but is only detected in that specific spectrum. Neither at pixel 60 (2010 datset), nor in the 2012, position 2 dataset, we do detect the absorption profile. Nontheless, the ion is clearly present in the environment close to the central star (in emission).

\subsection{Molecular emission lines}

\subsubsection{CO emission lines as temperature tracers}

The first and second overtone transitions of $\mathrm{CO}$ are detected and are used to determine the rotational temperature and column densities of $\mathrm{CO}$ in the Red Square Nebula. The bandheads of the $\Delta v=2$ of $\mathrm{CO}$, these are $\mathrm{CO}(0-2),(1-3)$, and (2-4) transitions, are identified at $2.29 \mu \mathrm{m}, 2.32 \mu \mathrm{m}$, and at $2.35 \mu \mathrm{m}$, respectively. We also identified the $\Delta v=3$ transitions of $\mathrm{CO}$, CO (0-3), (1-4), (2-5), (3-6), and (4-7) at around $1.56 \mu \mathrm{m}$, $1.58 \mu \mathrm{m}, 1.60 \mu \mathrm{m}, 1.62 \mu \mathrm{m}$, and $1.64 \mu \mathrm{m}$. The analysis of the observed emission features is explained in the following sections and the results are plotted in Figs. 12 and 13. We simulate the observational data using PGOPHER (Western 2013), a program for simulating the rotational structure of molecules. The program assumes LTE conditions. As input parameters for the PGOPHER simulation we used the HITEMP (High-Temperature Molecular Spectroscopic Database) datafiles (Rothman et al. 2010). Using these datafiles we simulated the CO spectrum for various temperatures and overplotted the observational spectrum with the simulated PGOPHER spectrum.

Figures 12 and 13 show a comparison between the observational data (black) and the simulated spectrum (red). Figure 12 shows the first overtone transition. The simulation is normalized to the intensity in the $\mathrm{CO}(0-2)$ bandhead of the observational dataset. Figure 13 shows the second overtone transition of $\mathrm{CO}$ and is normalized to the intensity of $\mathrm{CO}(3-6)$ transition. The rotational temperatures are increased from top to bottom, respectively.

\subsubsection{First overtone transitions of $\mathrm{CO}$}

The band profile and relative intensities of the $\mathrm{CO}(0-2)$ transitions are very sensitive to the gas temperature. Only for temperatures in excess of $1000 \mathrm{~K}$, the bandheads do start to become prominent (Fig. 12). Comparison of the observations with the simulations indicate a temperature of roughly $3000 \mathrm{~K}$. The uncertainty in the temperature is around $20 \%$. 


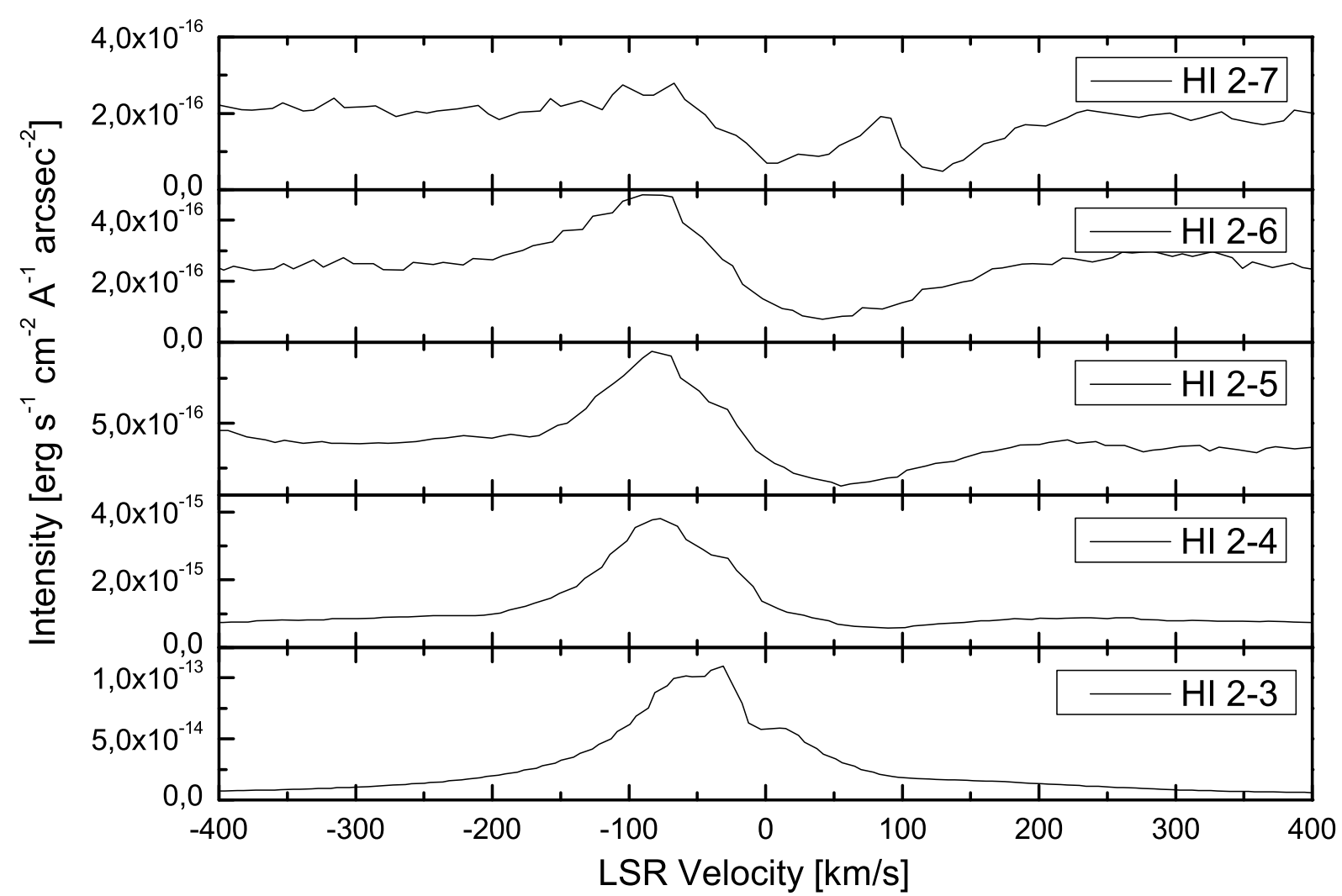

Fig. 7. H I line profiles. The wavelength is corrected for the local standard of rest (LSR $\left.=12.12 \mathrm{~km} \mathrm{~s}^{-1}\right)$. The zero point is equal to the wavelength $\left(\lambda_{\text {air }}\right)$ given from the NIST database. The figures show the transition of absorption features towards P Cygni line profiles.

\subsubsection{Second overtone transitions of $\mathrm{CO}$}

Figure 13 shows a zoom-in on the many weak emission lines in a very crowded part of the spectrum. Many of the second overtone transitions of $\mathrm{CO}$ are blended. However, the $\mathrm{CO}(3-6)$ transition lies free of other emission lines, and clearly exhibits rotational substructure. The simulation of the $\Delta v=3$ transition also suggests temperatures of about $3000 \mathrm{~K}$. Here, the uncertainty in the temperature is also in the order of $20 \%$.

\subsubsection{CO column density}

The CO column density for the various ro-vibrational transitions has been obtained. We integrated the emission bands $I_{\lambda}$ ( $\mathrm{erg} \mathrm{s}^{-1} \mathrm{~cm}^{-2} \mu \mathrm{m}^{-1} \operatorname{arcsec}^{-2}$ ) of the individual bandheads. The wavelength range over which the emission was integrated was chosen by comparison to the simulations at temperatures of $3000 \mathrm{~K}$. For $\Delta v=2$, for example, the simulation shows a clear and steep blue edge and a long degraded red tail. The flux can still be traced towards the steep blue edge of the next $\mathrm{CO}$ bandhead at longer wavelengths (i.e., at CO (1-3)). Hence, we integrated all fluxes in between two adjacent bandheads to account for the column density in each individual emission band of the $\Delta v=2$ transitions. However, for CO (2-4) and for the $\Delta v=3$ transitions, the situation is not as straight forward, due to low $\mathrm{S} / \mathrm{N}$ as well as blended atomic lines, respectively. Thus, the error bars depend on the $\mathrm{S} / \mathrm{N}$, the chosen baseline, and overlapping or blended emission lines. Here, the error in the integrated intensity is estimated to be in the order of $20 \%$ for individual unblended $\mathrm{CO}$ emission bands. For most lines in the $\Delta v=3$ overtone transition we adopted an error of 50\%, and an error of $20 \%$ for the CO (3-6) transition. Einstein $A$ coefficients were adopted from
Chandra et al. (1996) available through the vizier database for up to $J=150$. The rotational constants for the different vibrational levels were taken from Mishra et al. (2005). We then calculated the column density using

$N_{i}=\frac{4 \Pi \times I_{i}}{A_{i} \times h v}$

The results are given in Table 4. The table shows the flux for each ro-vibrational transition. The Einstein $A$ coefficients are also given. The resulting CO column density for each individual transition is shown, including the estimate on the error bars.

Figure 14 shows the relation between CO column density and upper level energy. A linear fit is used to determine the excitation temperature of $\mathrm{CO}$ in the Red Square. The excitation temperature is around $2100 \mathrm{~K}$ for the $\Delta v=2$ and $3200 \mathrm{~K}$ for the $\Delta v=3$ bands, respectively. The error on the linear fit is in the order of $50 \%$. These temperatures match within $1 \sigma$ with the $3000 \mathrm{~K}$ determined by the PGOPHER LTE simulations. From the integrated emission intensity we directly obtain the column densities of the various energy levels involved. We also determine a total column density of $\mathrm{CO}$ by using $3000 \mathrm{~K}$ as excitation temperature and extrapolating the column densities for the $v=0$ and $v=1$ transition by adopting the column density for the $\mathrm{CO}$ $(v=2)$ transition of $4.87 \times 10^{13} \mathrm{~cm}^{-2}$. This yields CO columns of $8.5 \times 10^{14}$ and $2.0 \times 10^{14} \mathrm{~cm}^{-2}$ for the $\mathrm{CO}(v=0)$ and $\mathrm{CO}$ $(v=1)$ vibrational levels, respectively. Adding up all the column densities from the various upper levels, and taking into account the extrapolated $\mathrm{CO}$ column densities for the ground and first vibrationally excited state, we obtain a total column density of $\mathrm{CO}$ of about $1.7 \times 10^{15} \mathrm{~cm}^{-2}$. The results are shown in Table 4 . 


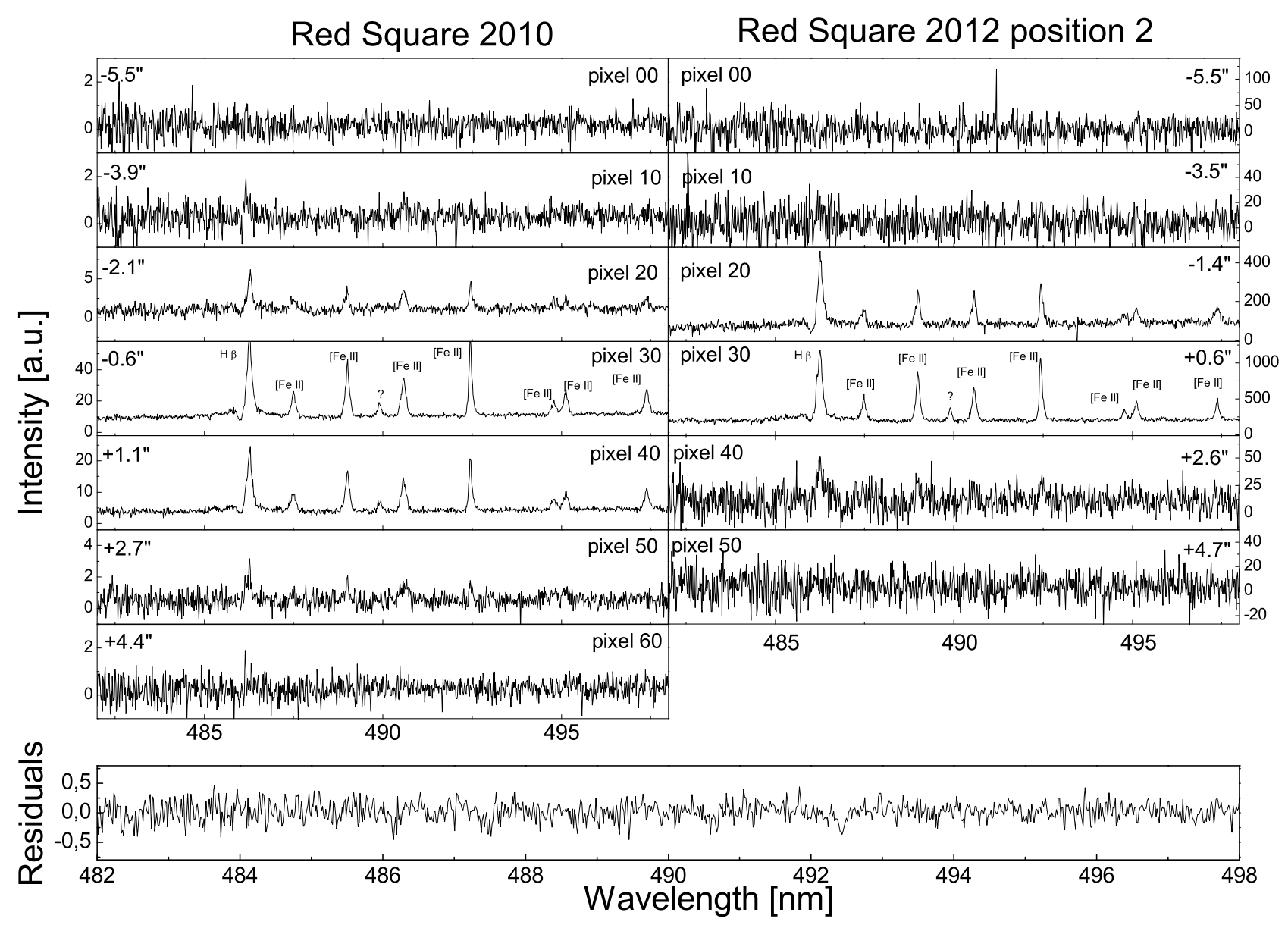

Fig. 8. $\mathrm{H} \beta$ line is shown for the spectrum taken in 2010 and for the spectrum taken in 2012, position 2 . The identified emission lines are labeled for better comparison. Pixel 00 (2010) corresponds to the south-east position. Pixel 00 for position 2 in 2012 is located in the east. Both spectra show clearly the $\mathrm{P}$ Cygni line profile of the $\mathrm{H} \beta$ line. Again, there is no difference in the individual line profiles for the different pixel spectra, and/or slit positions. There is also no change in relative intensity among the detected emission features. A clear decrease in intensity is detected with distance to the central star. The bottom-most panel gives the residuals of the slit spectrum in 2012 (position 2) when the spectrum at $+0.6^{\prime \prime}$ (pixel 30) is subtracted from the spectrum at $-2.5^{\prime \prime}$ (pixel 15) distance to the central star. Negative distances indicate directions in the south-(east) of the nebula. Positive distances indicate directions towards the north-(west).

\section{The Red Square and the Red Rectangle Nebula}

\subsection{Morphology}

Figure 15 shows a comparison of the morphology of both objects. The left panel shows the Red Square Nebula (Tuthill \& Lloyd 2007). The image has been taken using $H$-band adaptive optics from Mount Palomar. The right panel shows the Red Rectangle, optical image, taken with the HST PC and WF cameras applying different filters (Cohen et al. 2004). The similarities are striking, as both objects show the same X-shaped structure. In the Red Rectangle the circumbinary disk shapes the outflows into two conical lobes. The biconical outflows show regions of higher densities, perpendicular to the outflow direction, which have been named ladder-rungs. Most likely they are ring shaped features on the bicone surface. The origin of these features is unclear, but it has been suggested that these are caused by strong stellar pulsations leading to strong mass-loss events during stellar evolution in the binary system of the Red Rectangle Nebula. The X-shaped appearance, limbshaped paraboloids co-axial with the polar axis, and ladderrungs can also be found in the Red Square Nebula. The origin of these rungs is puzzling in the Red Square Nebula, as the central object is not confirmed to be a post-AGB object.
However, Miroshnichenko (2007) studied a wide range of objects that were classified as B[e] stars (Miroshnichenko 2007; Miroshnichenko et al. 2007). This classification was based on the presence of optical emission lines as well as IR excess. For many of their objects the evolutionary status is unknown, as it is for MWC 922, for which both pre- and post main sequence have been suggested. Based on these studies it has been suggested, that many B[e] objects belong to the class of FS CMa stars. In the same study, it is mentioned that likely all of these objects have a secondary companion (Miroshnichenko et al. 2007). Based on their findings, we speculate that the same processes that shape the nebula in HD 44179 is also operating in MWC 922. Our studies clearly indicate outflowing material, a disk in rotation around the central object(s) as well as shocked gas. In $H$-band images (Tuthill \& Lloyd 2007) the structure of the outflows is resolved and shows the same morphological similarities as seen in the Red Rectangle, as mentioned above. We argue that the Red Square Nebula is indeed formed by a binary system.

Soker (2015) has developed a detailed model for the origin of the various morphological characteristics of the Red Rectangle. In this model, the slowly expanding biconical structure of the Red Rectangle is formed by intermittent jets blown by the accreting companion. Many objects contain a pair of wineglass structures, symmetrically arranged with respect to a central disk, 


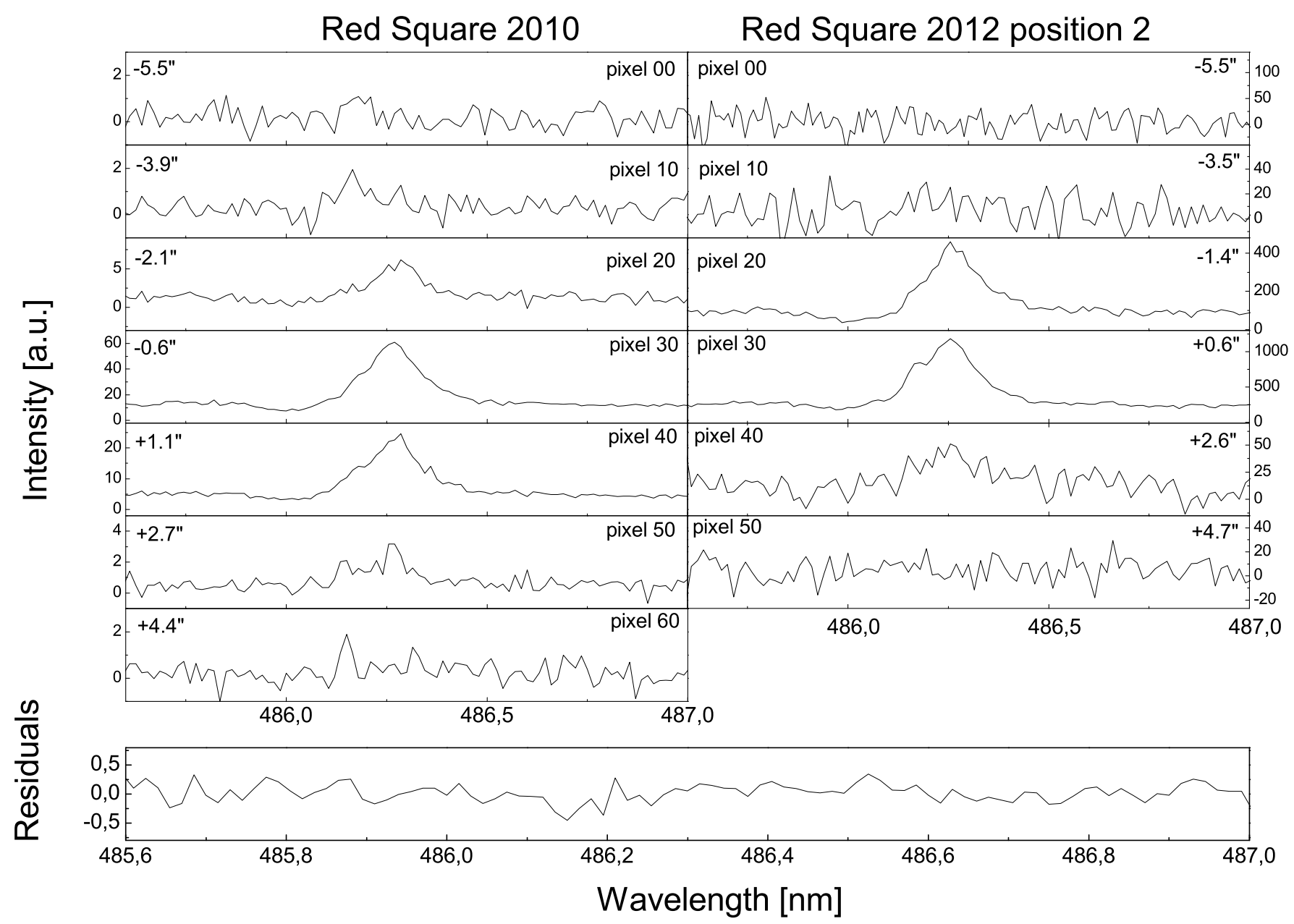

Fig. 9. Zoom-in on the $\mathrm{H} \beta$ line is shown for the spectrum taken in 2010 and for the spectrum taken in 2012, position 2. Both spectra show clearly the $\mathrm{P}$ Cygni line profile of the $\mathrm{H} \beta$ line. Again, there is no difference in the individual line profiles for the different pixel spectra, and/or slit positions. The bottom-most panel gives the residuals of the slit spectrum in 2012 (position 2) when the spectrum at $+0.6^{\prime \prime}$ (pixel 30) is subtracted from the spectrum at $-2.5^{\prime \prime}$ (pixel 15) distance to the central star. Negative distances indicate directions in the south-(east) of the nebula. Positive distances indicate directions towards the north-(west).

consisting of a ring connected by a paraboloid to a central object. Such a structure might be explained by a binary system where the primary object has a period of enhanced mass-loss forming a dense shell. Some of this material forms an accretion disk around the secondary and that leads to the launch of a jet. Interaction of this jet with the shell forms then the symmetric wineglass structures (Soker 2002). The Red Rectangle Nebula - and the Red Square Nebula - are special in that respect as they contain a set of those wineglass structures and this has been taken to imply an intermittent series of enhanced mass-loss periods in the primary object of the Red Rectangle and associated jet activity (Soker 2005). Given the morphological similarities, we argue that a similar scenario might be at work in the Red Square Nebula and there is some supporting evidence. While no companion has been directly or indirectly detected in MWC 922, the class of FS CMa objects is characterized by binarity. The mid IR excess heralds the presence of hot dust presumably in a circumstellar disk and that is supported by our analysis of the emission lines. Furthermore, Marston \& McCollum (2008) have interpreted the observed morphology in their deep $\mathrm{H} \alpha$ images as evidence for a one-sided jet in MWC 922. However, we note that this supposed jet is along the disk axis rather than the poles and it is not clear that this structure is a jet. An extended study of deep imaging of the Red Square Nebula might be very illuminating in this respect. While the morphologies and IR spectral signatures of the nebulae are very similar, there are also clear differences between the Red Rectangle and the Red Square. Specifically, the Red Rectangle contains an evolved luminous star, likely in a post-AGB evolutionary stage (Waelkens et al. 1996), while the primary star in MWC 922 is more massive and likely in an earlier evolutionary stage (Witt et al. 2009). So, in a way, the Red Square and the Red Rectangle are an astronomical example of convergent evolution where similar morphological characteristics are expressed by environmental pressures in very different objects.

\subsection{Comparison of the chemical components}

Despite the morphological similarities (disk, X-shaped outflows, including vortices and so-called ladder-rungs), we notice strong differences in the physical and chemical conditions of both sources. The reason for the difference in the physics and chemistry is based on the nature of the primary star. In the Red Rectangle Nebula, the central objects are a spectroscopic binary system, with a post-AGB star $(\simeq 8000 \mathrm{~K})$ feeding a main sequence 
Red Square 2010

Red Square 2012 position 2

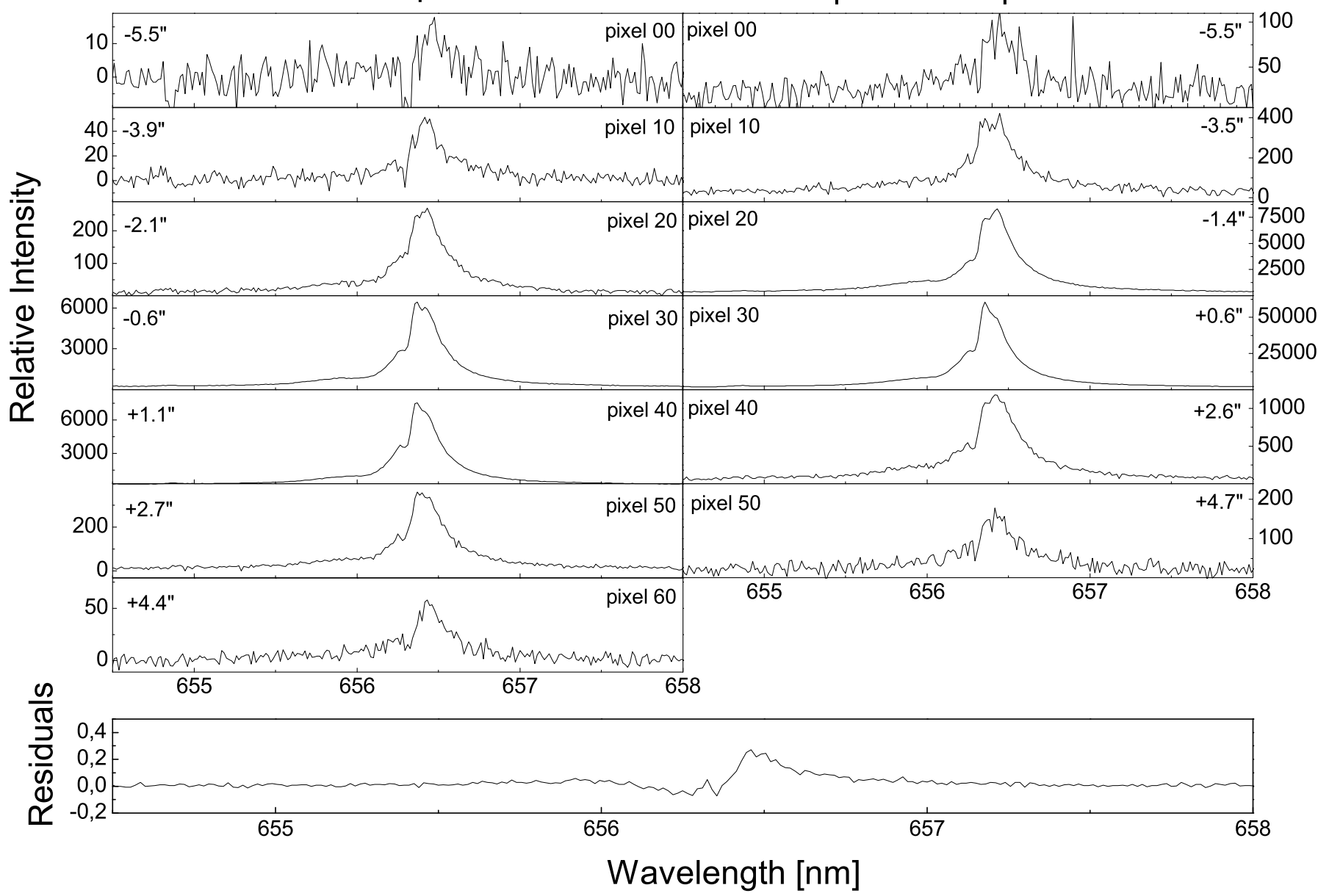

Fig. 10. $\mathrm{H} \alpha$ line is shown for the spectrum taken in 2010 and for the spectrum taken in 2012, position 2. Pixel 00 (2010) corresponds to the southeast position. Pixel 00 for position 2 in 2012 is located in the east. Here, actually a change in the line profile is visible: the absorption component at pixel 00 (south-east, in the 2010 dataset, and east for 2012) becomes weaker when going closer to the star (see pixel 30 at $-0.6^{\prime \prime}$ in 2010 and $+0.6^{\prime \prime}$ offset in 2012), whereas the emission component increases when going closer to the star. The bottom-most panel gives the residuals of the slit spectrum in 2012 (position 2) when the spectrum at $+0.6^{\prime \prime}$ (pixel 30) is subtracted from the spectrum at $-2.5^{\prime \prime}$ (pixel 15) distance to the central star. Here, the residuals are larger compared to the other residuals in the emission lines. This indicates that the $\mathrm{H} \alpha$ line is located on the pole of the star. Negative distances indicate directions in the south-(east) of the nebula. Positive distances indicate directions towards the north-(west).

star via Roche lobe overflow. The main sequence star shows signatures of an accretion disk and fast jets accelerated outwards, evident in a broad $\mathrm{H} \alpha$ component and CO UV emission lines (Witt et al. 2009). The bright red glow in the Red Rectangle in the optical part of the spectrum is caused by extended red emission (ERE), indicative of carbonaceous grains or silicate particles (Witt et al. 1998; Seahra \& Duley 1999; Ledoux et al. 2001; Malloci et al. 2004; Mulas et al. 2004; Chang et al. 2006; Berné et al. 2008). The bright red glow from the $H$-band adaptive optics image taken by Tuthill \& Lloyd (2007) is likely caused by scattered near-IR light off of the dust. The central objects in the Red Square are a B[e] star (FS CMa) with likely a secondary companion.

\subsection{The IR spectrum: PAHs and silicates}

PAHs (polycyclic aromatic hydrocarbons) and crystalline silicates have been detected in the near-IR and mid-IR spectrum of MWC 922 (Molster et al. 2002a,b,c; Peeters et al. 2002). Figure 16 shows the spectral energy distribution (SED) in the near- and mid IR of the Red Square Nebula in the upper panel.
The lower panel shows a comparison to the SED of the Red Rectangle Nebula. Both spectra cover the same spectral range. The PAHs emission features as well as the silicates have been labeled in both spectra for better comparison. We only distinguish between carbon based emission signatures, that is PAHs, and oxygen based emission features, that is silicates. For a more in-depth comparison and identification of the particular silicate features or PAHs please refer to Molster et al. (2002a,b,c) and Peeters et al. (2002).

Signatures of strong PAH emission are clearly visible between $1-10 \mu \mathrm{m}$ in both environments, as well as strong silicate emission bands at longer wavelengths, between 30 and $40 \mu \mathrm{m}$. It becomes obvious that the Red Rectangle possesses stronger PAH emission features, whereas the Red Square Nebula shows stronger silicate emission features. It was recognized that the Red Rectangle has a spatially separated chemistry with silicates located in the circumbinary disk that antedate the C-rich material in the nebula, in which PAHs have been detected (Van Winckel et al. 2002). This has been interpreted as the result of AGB evolution, in which the star is initially O-rich. The silicates are tracers of this O-rich phase, and are trapped in 


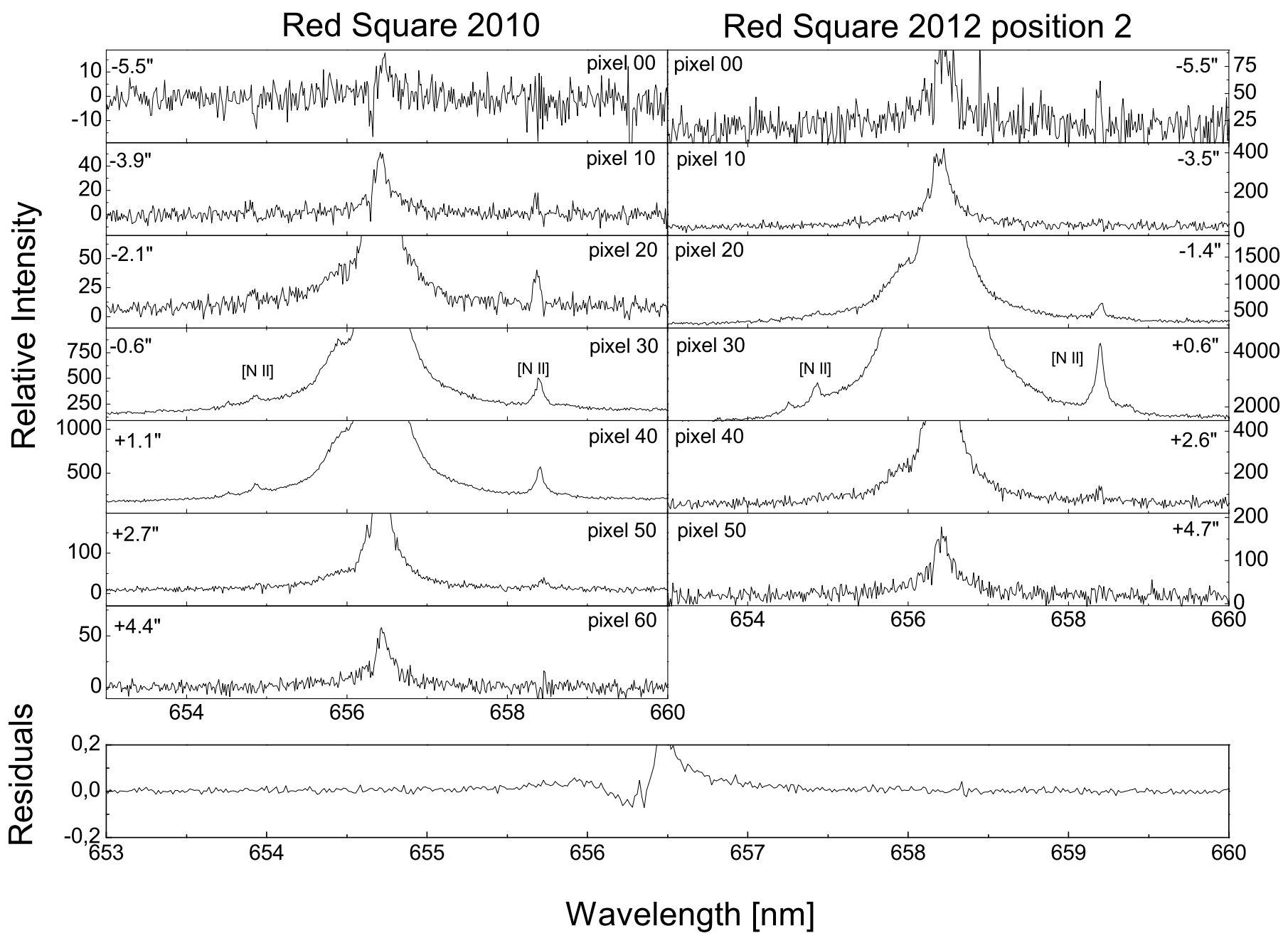

Fig. 11. Zoom-in on the [N II] lines located at $654.86 \mathrm{~nm}$ and $658.39 \mathrm{~nm}$, next to the $\mathrm{H} \alpha$ line. We plot the two spectra obtained in 2010 in 2012 , position 2. Pixel 00 (2010) corresponds to the south-east position. Pixel 00 for position 2 in 2012 is located in the east. The low density gas tracers can be seen in both spectra, but become very weak at the edges of the respective slit positions. However, a small absorption band can be detected at pixel 00, in the 2010 dataset. This is indicating that the forbidden N II can be seen close to the central star in emission (pixel 30, 2010 dataset), and further away from the central star in absorption (pixel 00, 2010 dataset). The bottom-most panel gives the residuals of the slit spectrum in 2012 (position 2) when the spectrum at +0.6" (pixel 30) is subtracted from the spectrum at -2.5" (pixel 15) distance to the central star. Negative distances indicate directions in the south-(east) of the nebula. Positive distances indicate directions towards the north-(west).

a (circumbinary) disk. Evolution turns the formerly O-rich star into a C-rich star, in which PAHs (and carbonaceous dust) are formed in the outflows.

In the Red Square Nebula, extended PAH emission has been detected (Lagadec et al. 2011), next to silicate emission features. This would imply that the PAHs must have been formed in an O-rich environment, hence either in the wind or the upper layers of the (circumbinary) disk, and then entrained into the wind, where then extended PAH emission has been detected (Lagadec et al. 2011). Besides the Red Rectangle Nebula, there are also many other objects in the literature which show $\mathrm{O}$ and C-rich environments, most of these objects however are postAGB objects (i.e., massive PNe or PNe with WC stars), and an explanation following the Red Rectangle is generally accepted. However, there are some other cases where O-rich and C-rich circumstellar compounds are present and a change in the $\mathrm{C} / \mathrm{O}$ ratio in the ejecta has been found. These include some massive red supergiants (Sylvester et al. 1994) as well as some LBVs (Voors et al. 2000; Guha Niyogi et al. 2014).

\subsection{The optical spectrum: molecules and atomic ions}

The optical spectrum in the Red Rectangle is dominated by broad ERE which is said to be indicative of large molecular emission stemming from either photo-luminescent processes of silicate particles or carbonaceous grains (Witt et al. 1998; Mulas et al. 2004; Wada et al. 2009). Superimposed on the ERE are narrow emission bands. In this region, two of the emission bands were identified as $C_{2}$ Swan-bands (Wehres et al. 2010). Some of these emission bands were also compared to the diffuse interstellar bands (DIBs), molecular features which are usually seen in absorption in diffuse interstellar clouds. The Red Rectangle seems unique in this respect as it shows the same molecular features (DIBs) seen in emission (Sarre 1991; Scarrott et al. 1992; Sarre et al. 1995; Van Winckel et al. 2002; Wehres et al. 2011). However, the Red Square nebula is lacking those unique emission features detected in the Red Rectangle environment, and DIBs are only seen in absorption. Most likely the DIBs are due to interstellar matter towards the line of sight 


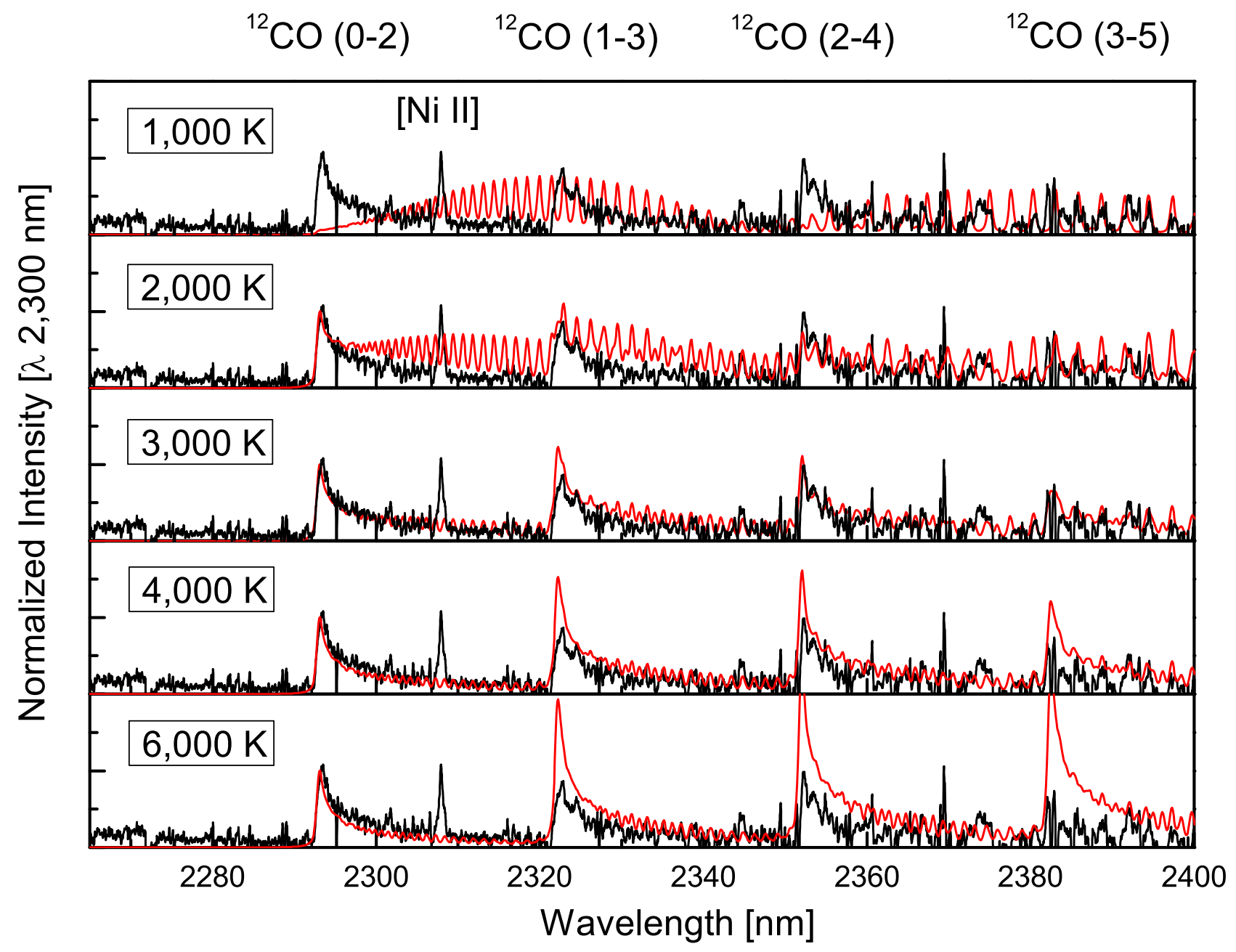

Fig. 12. Bandheads for the overtone transitions of the $\mathrm{CO}(0-2),(1-3)$ and $(2-4)$ vibrational transitions are shown. In addition we show the simulation for the next higher energetic transition $(\mathrm{CO}(3-5))$. The simulations are plotted in red on top of the observational data (black), assuming LTE conditions. The five panels - from top to bottom - show increasing temperatures. The intensities of the CO bandheads is reproduced best with a temperature of roughly $3000 \mathrm{~K}$.

of the Red Square, although one particular IR DIB has been argued to arise from circumstellar material in the nebula itself (Zasowski et al. 2015).

An overview of the X-shooter spectrum is given in Fig. 2. The ERE is completely absent and most of the emission lines could be identified coming from atoms or singly charged cations. Of these emission lines, most come from H I or Fe II emission lines. The only molecular species identified in the Red Square X-shooter spectra is CO. The ISO spectra however show emission line characteristics of PAHs and silicates. We also searched for molecular emission that previously has been identified in the Red Rectangle and/or in the ISM. In particular we looked for: $\mathrm{H}_{2}$ (Rosenberg et al. 2013), CN, CH, $\mathrm{C}_{2}$ (van Dishoeck \& Black 1989; Hobbs et al. 2004; Wehres et al. 2010), $\mathrm{CH}^{+}$(Carrington \& Ramsay 1982; Hobbs et al. 2004), $\mathrm{C}_{3}$ (Maier et al. 2001), NH (Hofzumahaus \& Stuhl 1985), $\mathrm{OH}^{+}$ (Loomis \& Brandt 1936), HCO (Scherer \& Rakestraw 1997), $\mathrm{H}_{2} \mathrm{O}^{+}$(Gan et al. 2004), SH (Ramsay 1952), $\mathrm{SH}^{+}$(Civiš et al. 1989), $\mathrm{CO}^{+}$(Wu et al. 2008; Vujisic \& Pesic 1988). It is intriguing that the Red Square Nebula shows no signature of molecular emission lines other than $\mathrm{CO}$, as well as emission lines of very large species such as PAHs and silicates. The Red Rectangle however, shows the whole spectrum from smaller to larger molecules.

\section{Conclusions and implications}

Most of the emission lines in the Red Square Nebula could be identified and a classification of the origin of the emission lines has been attempted, for instance, originating in a rotating, and dense disk, in the low density nebula, in a fast stellar wind, or in shocked gas. We summarize specific lines that are probes of different physical and chemical environments in MWC 922: firstly, the H I recombination lines probe the photosphere of the disk and also the extended nebula. Among those, the $\mathrm{H} \beta$ and $\mathrm{H} \alpha$ lines show a clear P Cygni profile probing the fast outflowing shell of gas in the nebula. The narrow cores of the [Fe II] lines are likely to originate in the disk photosphere but the broad [Fe II] emission component is likely associated with shocks in the outflow cones, probing stellar winds. Extended, nebular [O I] on the other hand is likely associated with shocks. According to Hollenbach \& McKee (1989) is the combination of [O I] and [Fe II] lines a good tracer of shocks in general. In addition, the [O I] line measures the ionization zone that separates the ionized photosphere from the underlying, molecule-rich dusty disk. In the disk we find the Ca II emission lines, exhibiting a clear double peaked profile, indicative of a disk in rotation. The disk is also rich in PAH and silicate emission features. Lastly, the [N II] 


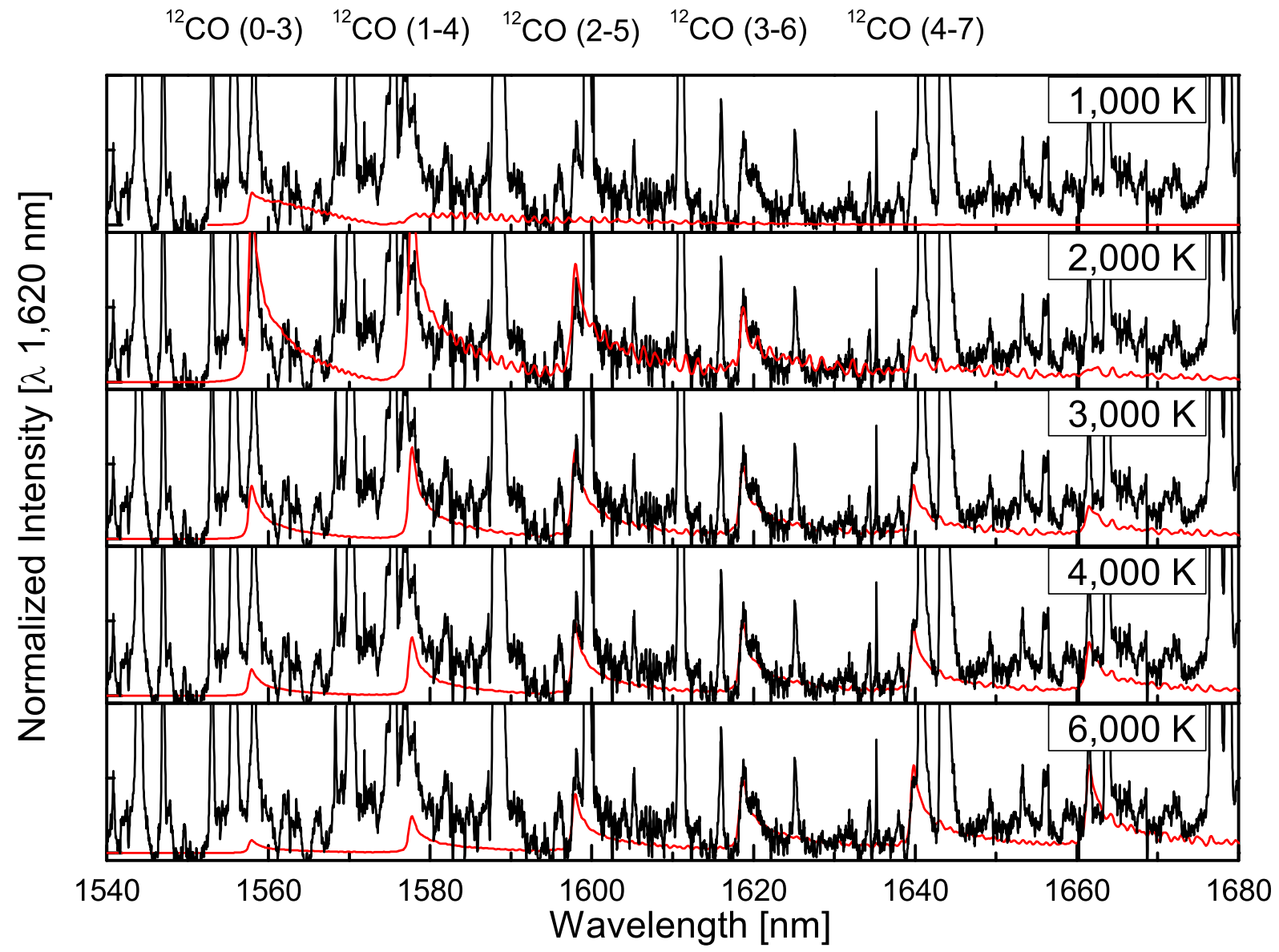

Fig. 13. Bandheads for $\mathrm{CO}(0-3),(1-4),(2-5),(3-6)$, and (4-7) vibrational bands are detected. This is based on the definite identification of $\mathrm{CO}(3-6)$ at $1620 \mathrm{~nm}$. The simulations are plotted in red on top of the observational data (black), assuming LTE conditions. A temperature of roughly $3000 \mathrm{~K}$ in the simulation matches the observational band strengths best.

Table 4. CO column densities.

\begin{tabular}{l|c|c|c}
\hline \hline Transition & $\mathrm{F}_{\lambda}\left(\mathrm{erg} \mathrm{s}^{-1} \mathrm{~cm}^{-2} \operatorname{arcsec}^{2}\right)$ & Einstein $A\left(\mathrm{~s}^{-1}\right)$ & Column density $\left(\mathrm{cm}^{-2}\right)$ \\
\hline \multicolumn{4}{c}{$\Delta v=2$} \\
\hline $\mathrm{CO}(0-2)$ & $6.79 \mathrm{E}-12 \pm 1.4 \mathrm{E}-12$ & $5.5 \mathrm{E}-1$ & $4.87 \mathrm{E} 13 \pm 9.7 \mathrm{E} 12$ \\
$\mathrm{CO}(1-3)$ & $9.86 \mathrm{E}-12 \pm 2 \mathrm{E}-12$ & 1.63 & $2.43 \mathrm{E} 13 \pm 4.9 \mathrm{E} 12$ \\
$\mathrm{CO}(2-4)$ & $2.27 \mathrm{E}-12 \pm 4.5 \mathrm{E}-11$ & 3.21 & $2.87 \mathrm{E} 12 \pm 5.7 \mathrm{E} 11$ \\
\hline \multicolumn{4}{c}{$\Delta v=3$} \\
\hline $\mathrm{CO}(0-3)$ & $6.94 \mathrm{E}-13 \pm 3.5 \mathrm{E}-13$ & $8 \mathrm{E}-3$ & $3.68 \mathrm{E} 13 \pm 1.8 \mathrm{E} 13$ \\
$\mathrm{CO}(1-4)$ & $4.41 \mathrm{E}-13 \pm 2.2 \mathrm{E}-13$ & $3.4 \mathrm{E}-2$ & $4.16 \mathrm{E} 12 \pm 2.1 \mathrm{E} 12$ \\
$\mathrm{CO}(2-5)$ & $6.31 \mathrm{E}-13 \pm 3.1 \mathrm{E}-13$ & $8.9 \mathrm{E}-2$ & $3.07 \mathrm{E} 12 \pm 1.5 \mathrm{E} 12$ \\
$\mathrm{CO}(3-6)$ & $5.71 \mathrm{E}-13 \pm 2.9 \mathrm{E}-13$ & $1.77 \mathrm{E}-1$ & $1.42 \mathrm{E} 12 \pm 2.8 \mathrm{E} 11$ \\
\hline \multicolumn{4}{c}{ Extrapolated CO column densities } \\
\hline $\mathrm{CO} v=0$ & \multicolumn{3}{c}{$8.5 \mathrm{E} 14 \pm 1.7 \mathrm{E} 14$} \\
$\mathrm{CO} v=1$ & \multicolumn{4}{c}{$2.0 \mathrm{E} 14 \pm 4.0 \mathrm{E} 13$} \\
Total CO & \multicolumn{4}{c}{$1.7 \mathrm{E} 15 \pm 3.4 \mathrm{E} 14$} \\
\hline
\end{tabular}

and [S II] forbidden lines originate in low density, but fairly hot nebular gas.

In Fig. 17 we present a schematic on the Red Square Nebula showing the detected emission lines and their origin within the Red Square Nebula. Extended PAH emission is shown. In the disk we also locate $\mathrm{Ca}$ II lines and $\mathrm{CO}$ band emission, as well as $\mathrm{HI}$ and $\mathrm{OI}$. H I is found in the photosphere of the disk, whereas
O I traces the ionization zone separating the ionized disk gas from the underlying molecular zone. The plethora of forbidden lines, including the [Fe II], [N II], [S II] as well as [Ca II], and other trace elements, are good tracers of the low density nebular environment. Plenty of the Fe II lines show a broad emission base, indicating fast stellar winds. In total, four of the H I recombination lines that are detected show $\mathrm{P}$ Cygni profiles, again 
N. Wehres et al.: The Red Square Nebula

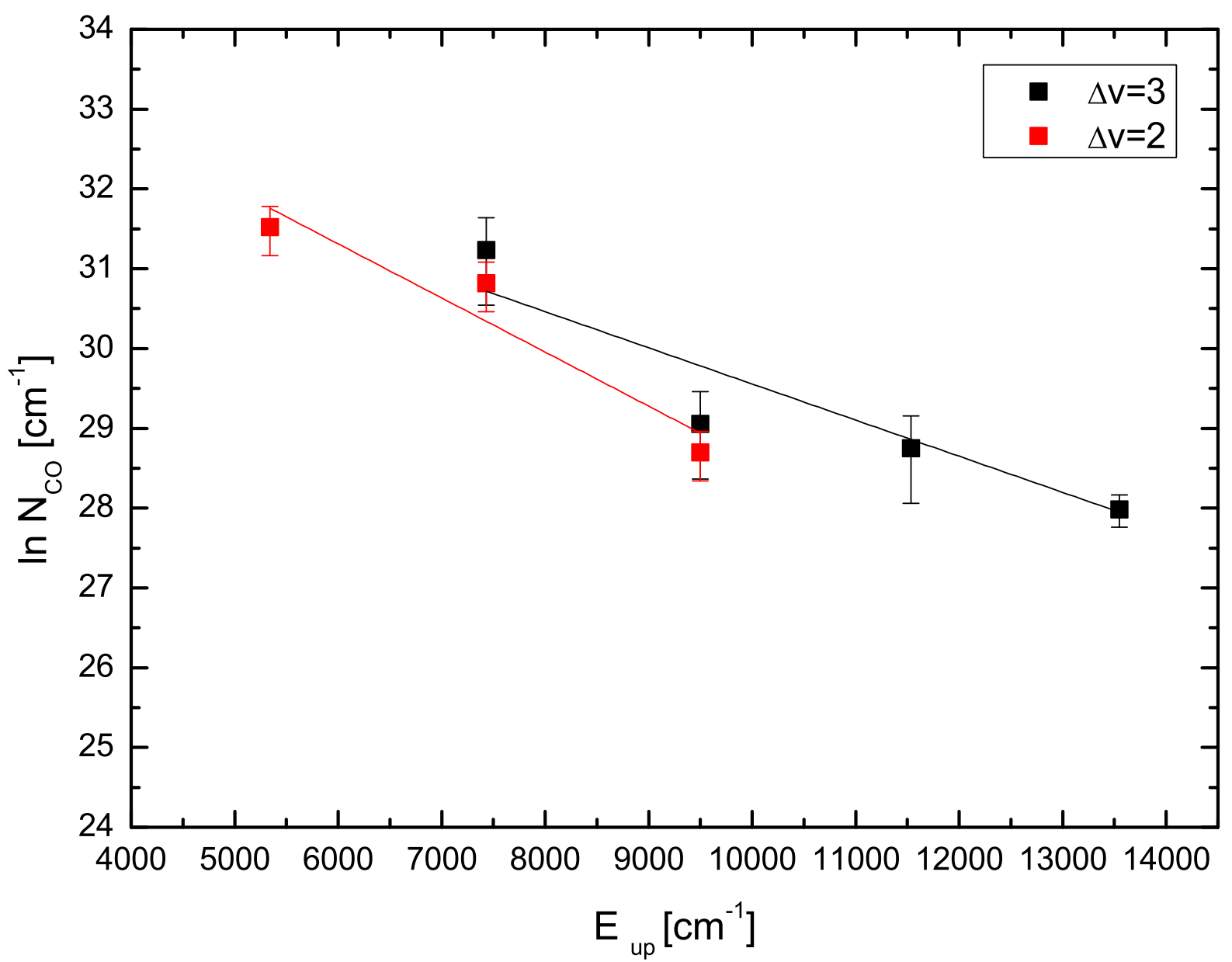

Fig. 14. Rotation diagram for the two CO overtone transitions $\Delta v=2$ and $\Delta v=3$. The resulting temperatures are 2140 and $3200 \mathrm{~K}$.

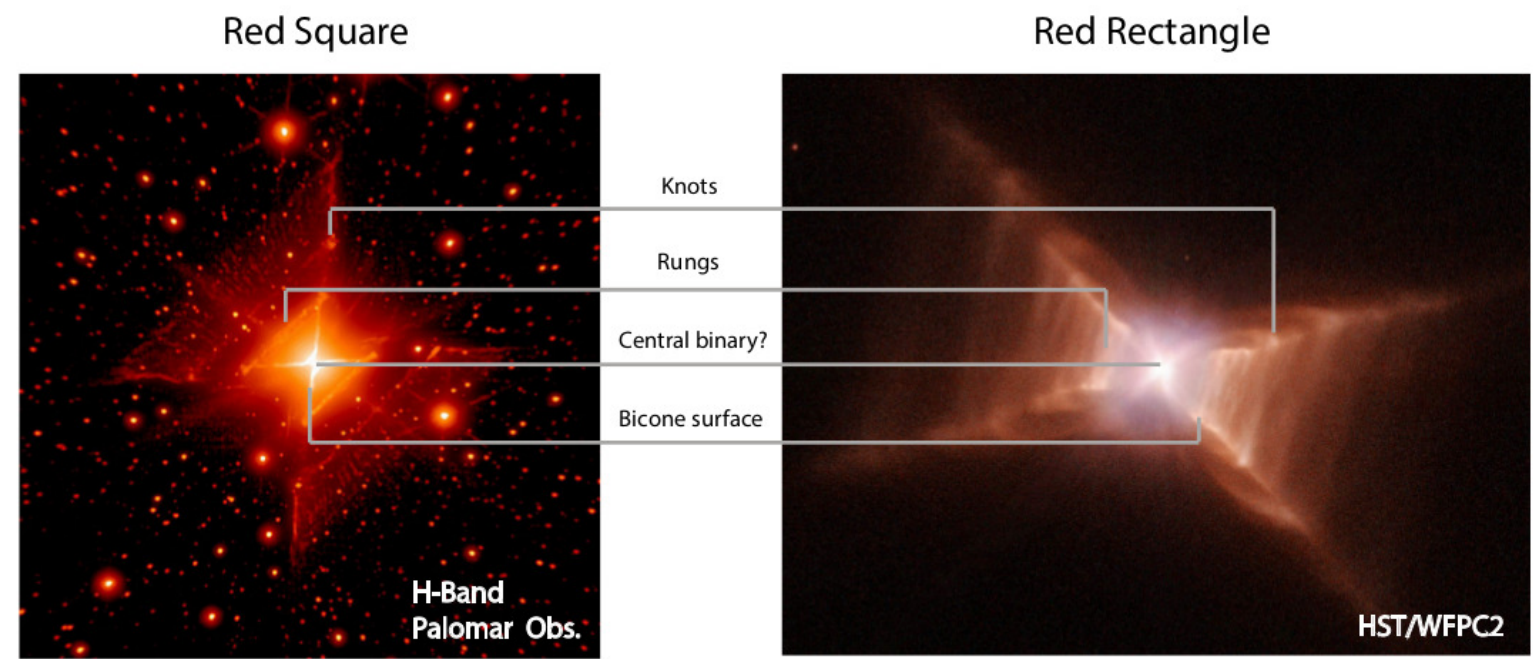

Fig. 15. Left panel: Red Square Nebula from Mt. Palomar H-band adaptive optics (Tuthill \& Lloyd 2007). Right panel: Red Rectangle Nebula using the HST PC and WF cameras applying different filters (Cohen et al. 2004). The figure highlights the similarities in morphology between these two striking objects.

indicative of outflowing material in an expanding shell of gas. We conclude that the physical and chemical conditions in the Red Rectangle and Red Square Nebula are very distinct, despite the similar morphology, such as the ladder-rungs in the outflowing material, the X-shaped bicones, the placement of knots, and the detailed comblike structures at the termination of the outflow cones. However, we argue that the forces driving the morphology of the Red Square are similar to those in the Red Rectangle Nebula. We speculate that, like the Red Rectangle, the Red Square Nebula also consists of a binary star system. This interaction would explain the formation of a cicumbinary disk and the outflow cones, including the ladder-rung structures. 


\section{Red Square}

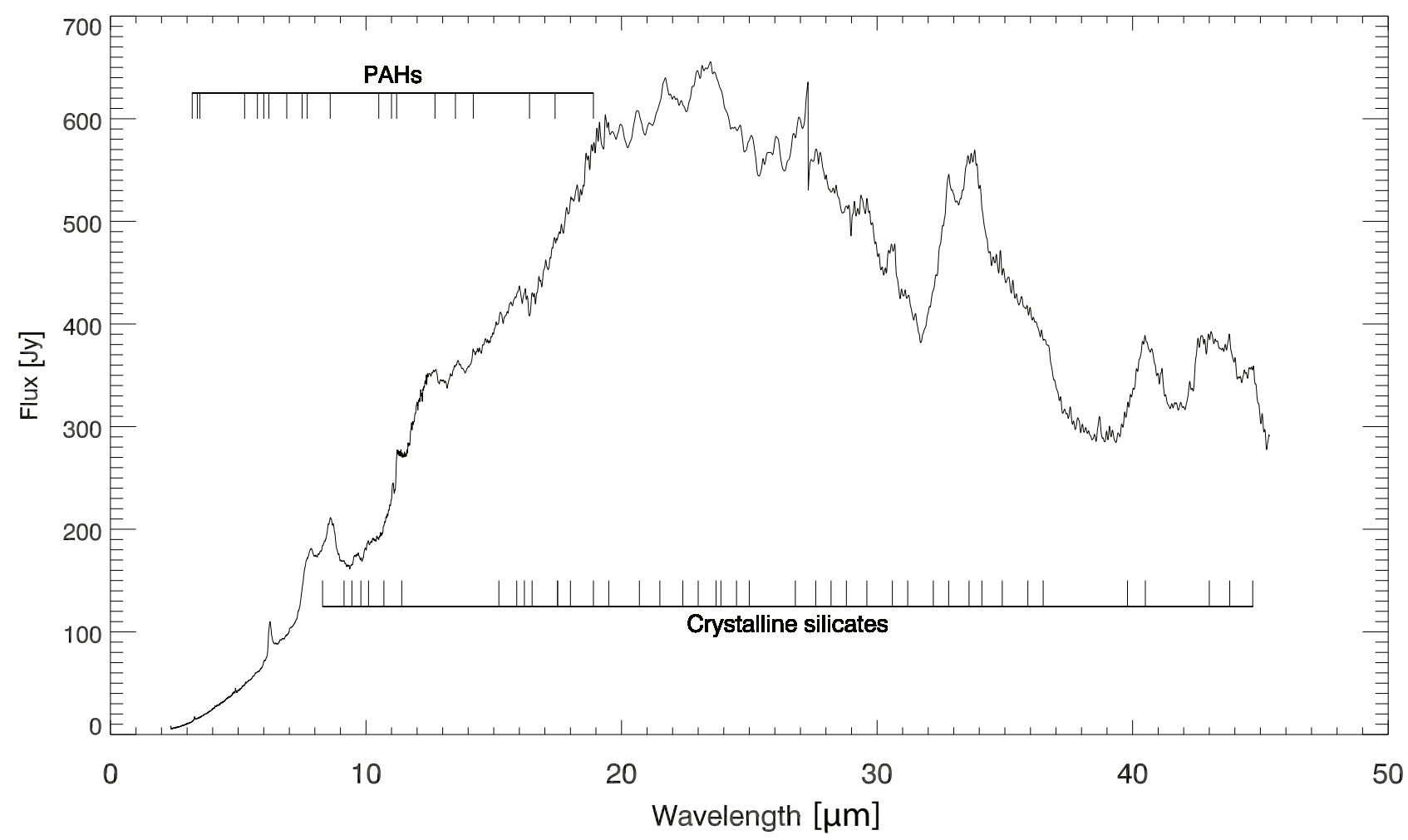

Red Rectangle

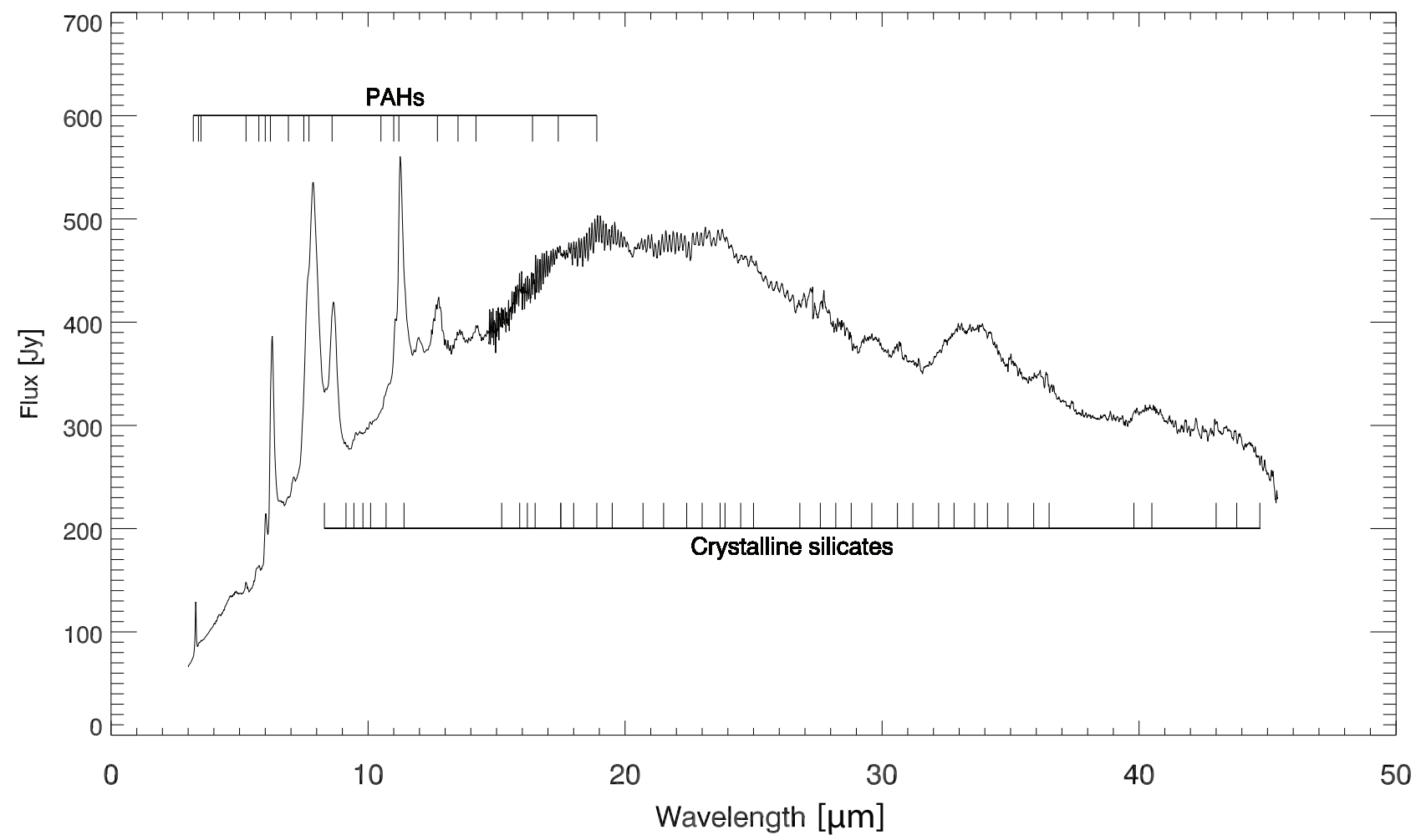

Fig. 16. Comparison of the near-IR - mid-IR ISO spectrum of the Red Square Nebula (upper panel) and the Red Rectangle Nebula (lower panel). The Red Square shows stronger emission features of crystalline silicates, whereas the Red Rectangle shows stronger emission of PAHs (polycyclic aromatic hydrocarbons). 


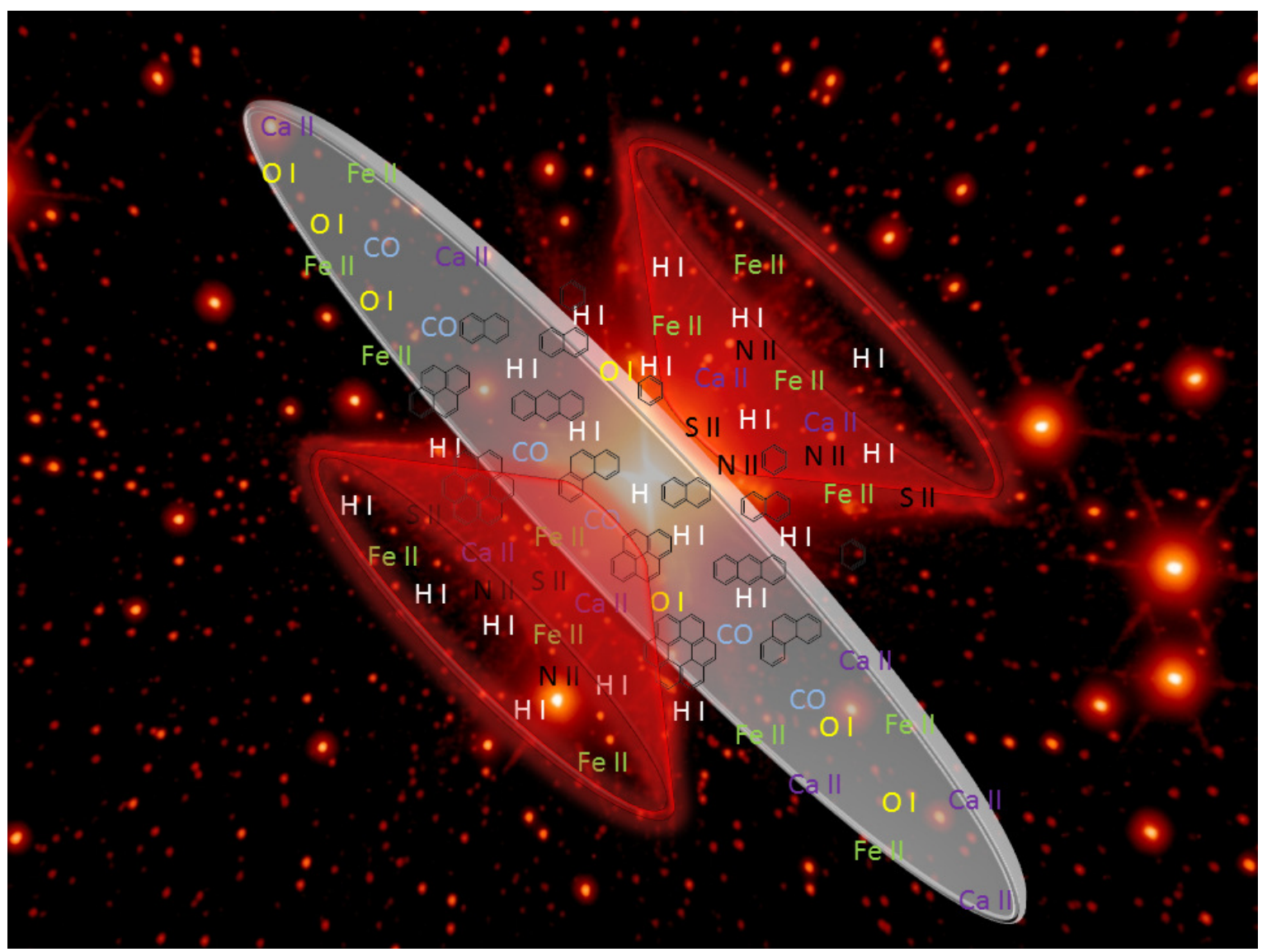

Fig. 17. Identified emission lines are depicted schematically. The Red Square image has been taken by Tuthill \& Lloyd (2007). According to our analysis we place the identified emission features according to their location in the nebula.

Acknowledgements. Based on observations collected at the European Organisation for Astronomical Research in the Southern Hemisphere under ESO program IDs 385.C-072 and 089.C-0874. The research is carried out within the Collaborative Research Centre 956, sub-project [B3], funded by the Deutsche Forschungsgemeinschaft (DFG). We also would like to thank the referee for insightful comments that helped to improve the paper.

\section{References}

Allen, D. A., \& Swings, J. P. 1976, A\&A, 47, 293 Andrillat, Y., \& Swings, J. P. 1976, ApJ, 204, L123 Aret, A., \& Kraus, M. 2015, EAS Pub. Ser., 71, 231

Aret, A., Kraus, M., Muratore, M. F., \& Borges Fernandes, M. 2012, MNRAS, 423, 284

Berné, O., Joblin, C., Rapacioli, M., et al. 2008, A\&A, 479, L41

Cardelli, J. A., Clayton, G. C., \& Mathis, J. S. 1989, ApJ, 345, 245

Carrington, A., \& Ramsay, D. A. 1982, Phys. Scr., 25, 272

Chandra, S., Maheshwari, V. U., \& Sharma, A. K. 1996, A\&AS, 117, 557

Chang, H.-C., Chen, K., \& Kwok, S. 2006, ApJ, 639, L63

Civiš, S., Blom, C. E., \& Jensen, P. 1989, J. Mol. Spectr., 138, 69

Cohen, M., Van Winckel, H., Bond, H. E., \& Gull, T. R. 2004, AJ, 127, 2362

Cox, N. L. J., Cami, J., Kaper, L., et al. 2014, A\&A, 569, A117

Cushing, M. C., Vacca, W. D., \& Rayner, J. T. 2004, PASP, 116, 362

Draine, B. T. 2003, ARA\&A, 41, 241

Ehrenfreund, P., Cami, J., Dartois, E., \& Foing, B. H. 1997, A\&A, 318, L28

Gan, Y., Yang, X., Guo, Y., et al. 2004, Mol. Phys., 102, 611

Garstang, R. H. 1962, MNRAS, 124, 321

Geballe, T. R., Najarro, F., Figer, D. F., Schlegelmilch, B. W., \& de La Fuente, D. 2011, Nature, 479, 200

Guha Niyogi, S., Min, M., Meixner, M., et al. 2014, A\&A, 569, A80

Hamann, F. 1994, ApJS, 93, 485

Hobbs, L. M., Thorburn, J. A., Oka, T., et al. 2004, ApJ, 615, 947

Hobbs, L. M., York, D. G., Thorburn, J. A., et al. 2009, ApJ, 705, 32

Hofzumahaus, A., \& Stuhl, F. 1985, J. Chem. Phys., 82, 5519
Hollenbach, D., \& McKee, C. F. 1989, ApJ, 342, 306

Israelian, G., \& de Groot, M. 1999, Space Sci. Rev., 90, 493

Kramida, A., Ralchenko, Yu., Reader, J., \& NIST ASD Team 2013, NIST Atomic Spectra Database (ver. 5.1), http://physics.nist.gov/asd [2014, August 8], National Institute of Standards and Technology, Gaithersburg, MD Lagadec, E., Verhoelst, T., Mékarnia, D., et al. 2011, MNRAS, 417, 32

Lamers, H. J. G. L. M., Zickgraf, F.-J., de Winter, D., Houziaux, L., \& Zorec, J. 1998, A\&A, 340, 117

Ledoux, G., Guillois, O., Huisken, F., et al. 2001, A\&A, 377, 707

Lenzen, R., Hartung, M., Brandner, W., et al. 2003, in Instrument Design and Performance for Optical/Infrared Ground-based Telescopes, eds. M. Iye, \& A. F. M. Moorwood, Proc. SPIE, 4841, 944

Loomis, F. W., \& Brandt, W. H. 1936, Phys. Rev., 49, 55

Maier, J. P., Lakin, N. M., Walker, G. A. H., \& Bohlender, D. A. 2001, ApJ, 553, 267

Malloci, G., Mulas, G., \& Benvenuti, P. 2004, A\&A, 420, 809

Marston, A. P., \& McCollum, B. 2008, A\&A, 477, 193

Miroshnichenko, A. S. 2007, ApJ, 667, 497

Miroshnichenko, A. S., Manset, N., Kusakin, A. V., et al. 2007, ApJ, 671, 828

Mishra, A. P., Shetty, B. J., \& Kshirsagar, R. J. 2005, J. Mol. Spectr., 232, 296

Molster, F. J., Waters, L. B. F. M., \& Tielens, A. G. G. M. 2002a, A\&A, 382, 222

Molster, F. J., Waters, L. B. F. M., Tielens, A. G. G. M., \& Barlow, M. J. 2002b, A\&A, 382, 184

Molster, F. J., Waters, L. B. F. M., Tielens, A. G. G. M., Koike, C., \& Chihara, H. 2002c, A\&A, 382, 241

Mulas, G., Malloci, G., \& Benvenuti, P. 2004, A\&A, 420, 921

Nussbaumer, H., \& Storey, P. J. 1980, A\&A, 89, 308

Nussbaumer, H., \& Storey, P. J. 1988, A\&A, 193, 327

Peeters, E., Hony, S., Van Kerckhoven, C., et al. 2002, A\&A, 390, 1089

Pesenti, N., Dougados, C., Cabrit, S., et al. 2003, A\&A, 410, 155

Ramsay, D. A. 1952, J. Chem. Phys., 20, 1920

Reipurth, B., Bally, J., Graham, J. A., Lane, A. P., \& Zealey, W. J. 1986, A\&A, 164,51

Rosenberg, M. J. F., van der Werf, P. P., \& Israel, F. P. 2013, A\&A, 550, A12

Rothman, L. S., Gordon, I. E., Barber, R. J., et al. 2010, J. Quant. Spectr. Rad. Transf., 111, 2139 
Rousset, G., Lacombe, F., Puget, P., et al. 2003, in Adaptive Optical System Technologies II, eds. P. L. Wizinowich, \& D. Bonaccini, Proc. SPIE, 4839, 140

Rudy, R. J., Erwin, P., Rossano, G. S., \& Puetter, R. C. 1992, ApJ, 398, 278

Sarre, P. J. 1991, Nature, 351, 356

Sarre, P. J., Miles, J. R., Kerr, T. H., et al. 1995, MNRAS, 277, L41

Scarrott, S. M., Watkin, S., Miles, J. R., \& Sarre, P. J. 1992, MNRAS, 255, 11P

Scherer, J. J., \& Rakestraw, D. J. 1997, Chem. Phys. Lett., 265, 169

Seahra, S. S., \& Duley, W. W. 1999, ApJ, 520, 719

Soker, N. 2002, ApJ, 577, 839

Soker, N. 2005, AJ, 129, 947

Soker, N. 2015, ApJ, 800, 114

Sylvester, R. J., Barlow, M. J., \& Skinner, C. J. 1994, MNRAS, 266, 640

The, P. S., de Winter, D., \& Perez, M. R. 1994, A\&AS, 104, 315

Tuairisg, S. Ó., Cami, J., Foing, B. H., Sonnentrucker, P., \& Ehrenfreund, P. 2000, A\&AS, 142, 225

Tuthill, P. G., \& Lloyd, J. P. 2007, Science, 316, 247

Vacca, W. D., Cushing, M. C., \& Rayner, J. T. 2003, PASP, 115, 389

van Dishoeck, E. F., \& Black, J. H. 1989, ApJ, 340, 273

van Hoof, P. 2014, The Atomic Line List v2.04, http://www.pa.uky.edu/ peter/atomic/
Van Winckel, H., Cohen, M., \& Gull, T. R. 2002, A\&A, 390, 147

Voors, R. H. M. 1999, Ph.D. Thesis, Universiteit Utrecht, The Netherlands

Voors, R. H. M., Waters, L. B. F. M., de Koter, A., et al. 2000, A\&A, 356, 501

Vujisic, B. R., \& Pesic, D. S. 1988, J. Mol. Spectr., 128, 334

Wada, S., Mizutani, Y., Narisawa, T., \& Tokunaga, A. T. 2009, ApJ, 690, 111

Waelkens, C., Van Winckel, H., Waters, L. B. F. M., \& Bakker, E. J. 1996, A\&A, 314, L17

Wehres, N., Romanzin, C., Linnartz, H., van Winckel, H., \& Tielens, A. G. G. M. 2010, A\&A, 518, A36

Wehres, N., Linnartz, H., van Winckel, H., \& Tielens, A. G. G. M. 2011, A\&A, 533, A28

Wehres, N., Ochsendorf, B., Bally, J., et al. 2014, in IAU Symp., 297, eds. J. Cami, \& N. L. J. Cox, 226

Western, C. M. 2013, PGOPHER, a Program for Simulating Rotational Structure, University of Bristol, http://pgopher.chm.bris.ac.uk

Witt, A. N., Gordon, K. D., \& Furton, D. G. 1998, ApJ, 501, L111

Witt, A. N., Vijh, U. P., Hobbs, L. M., et al. 2009, ApJ, 693, 1946

Wu, Y., Yang, X., Guo, Y., \& Chen, Y. 2008, J. Mol. Spectr., 248, 81

Zasowski, G., Chojnowski, S. D., Whelan, D. G., et al. 2015, ApJ, 811, 119 


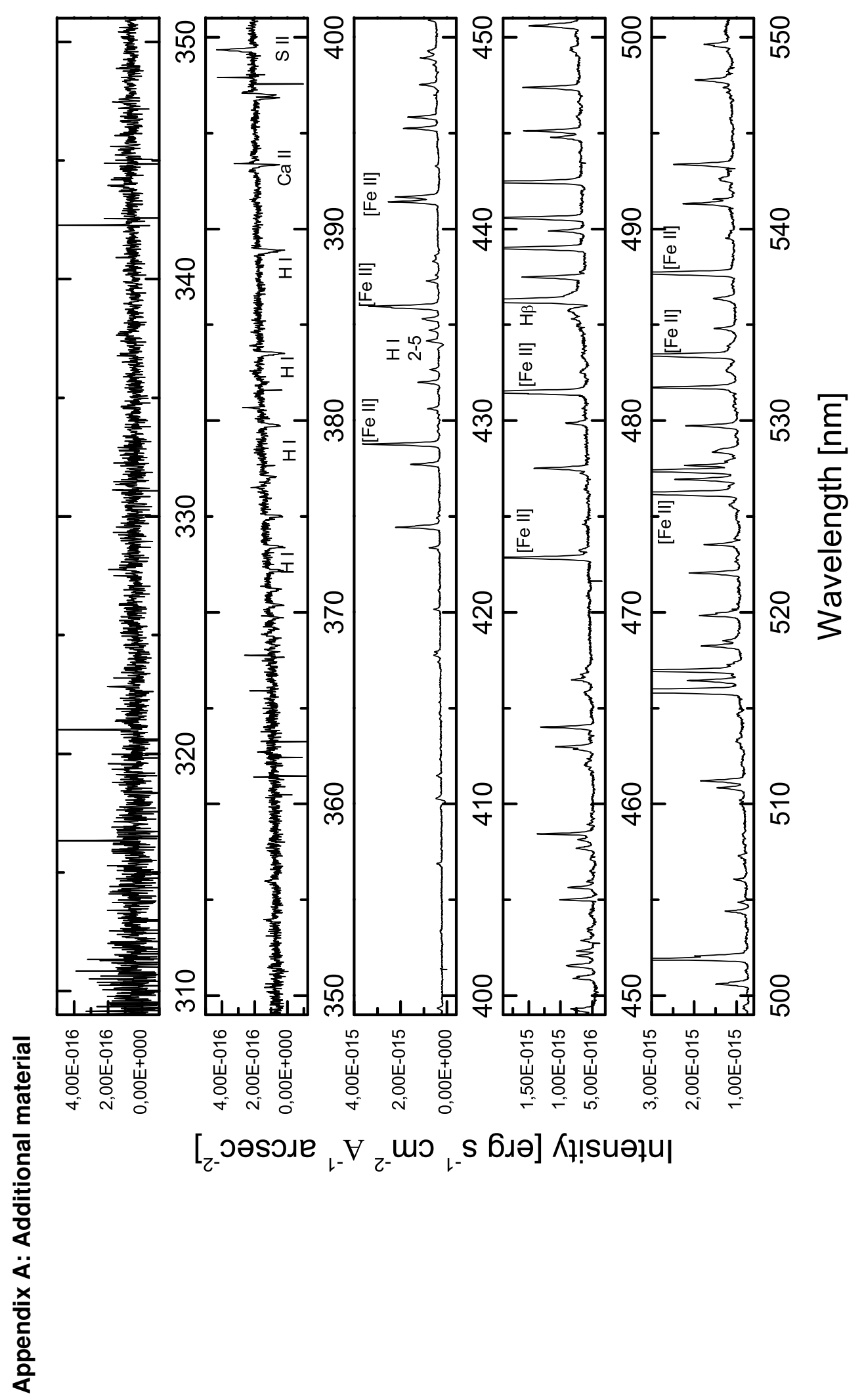




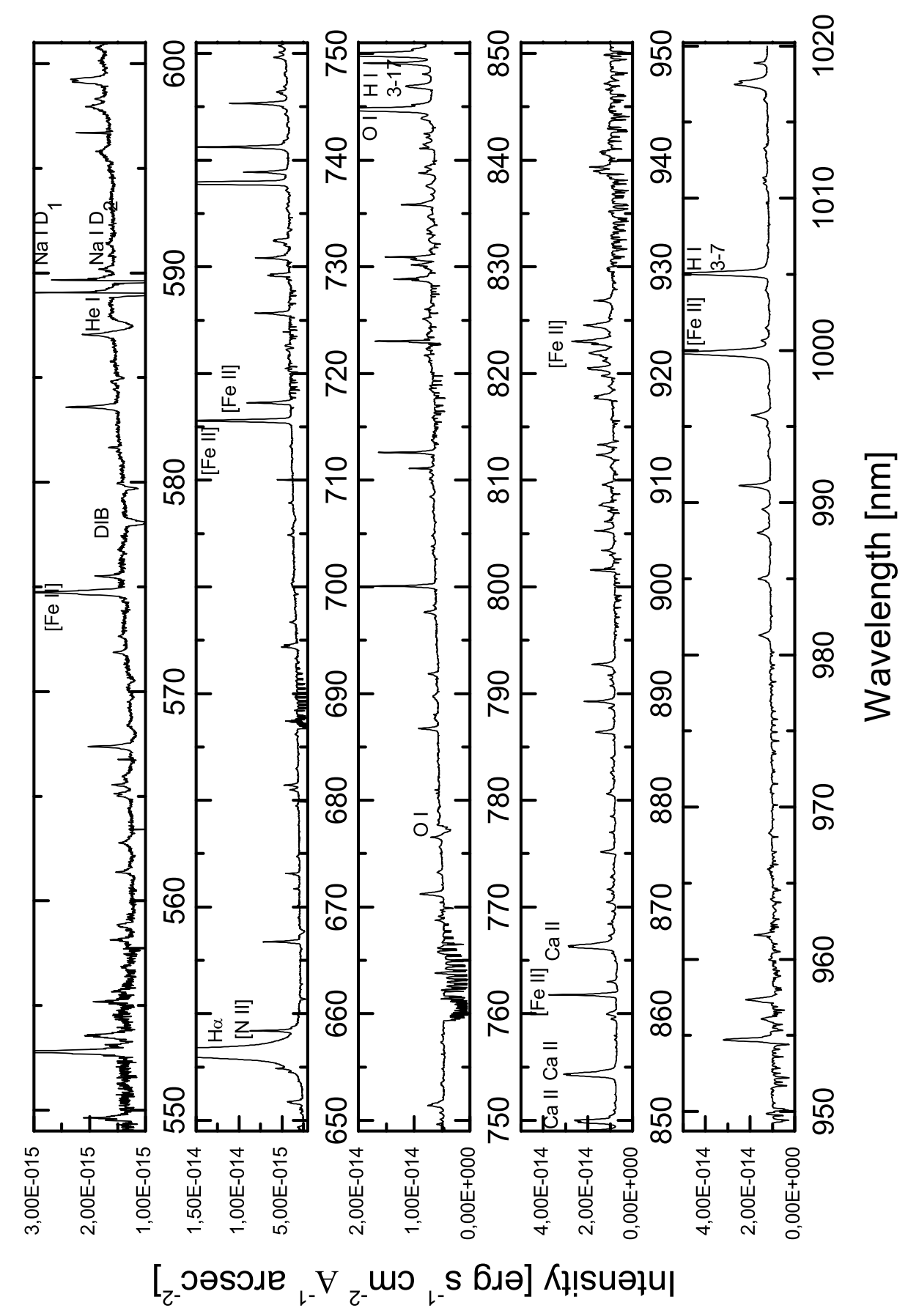




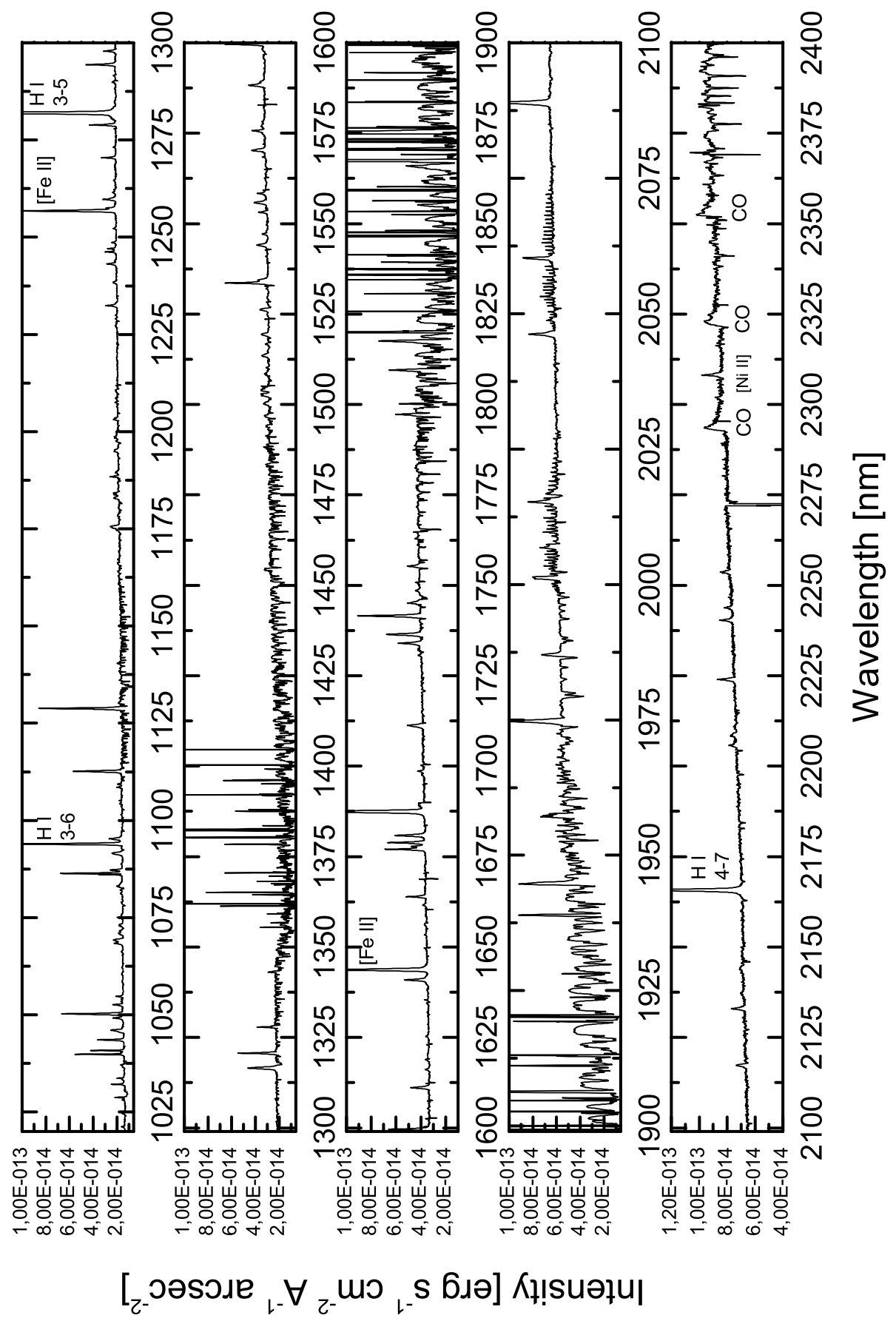




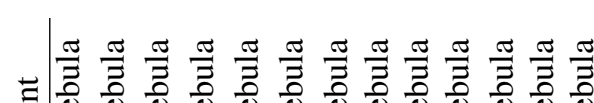

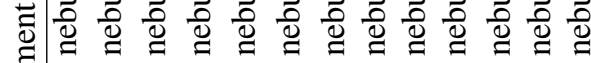

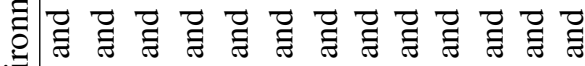

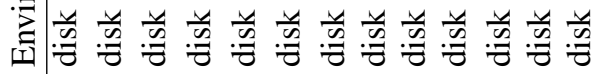

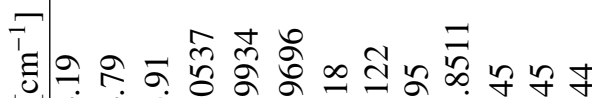

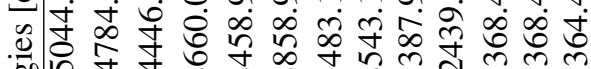
紫字寺寺

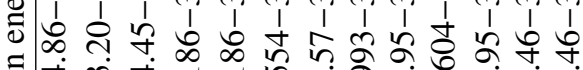
过过

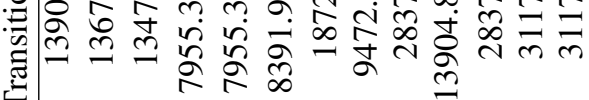

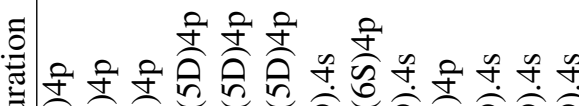

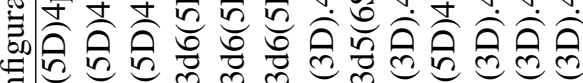
要

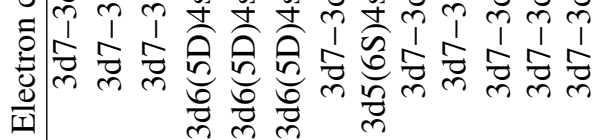

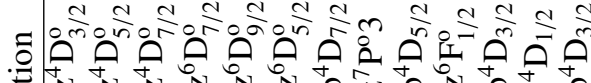

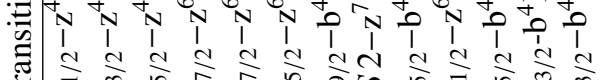

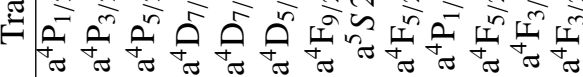

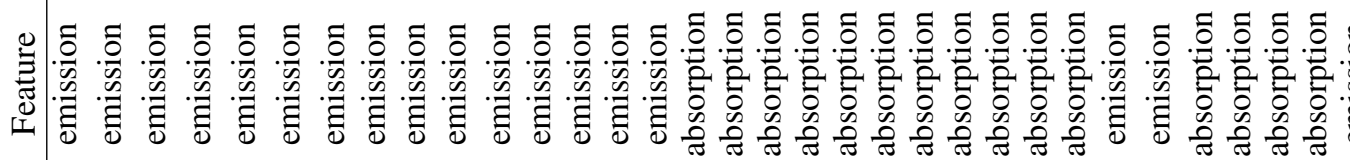

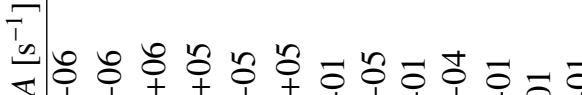

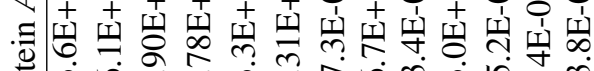
竞

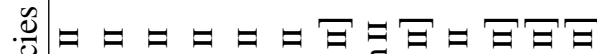

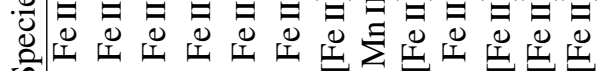

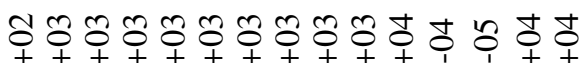

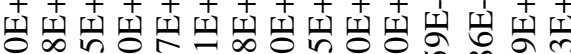

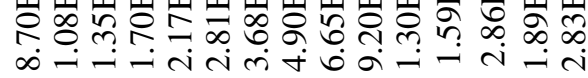

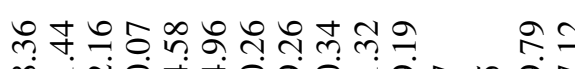

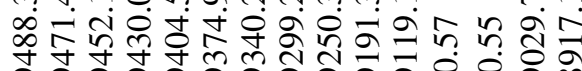

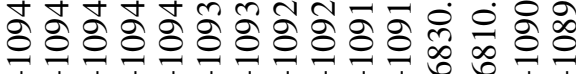

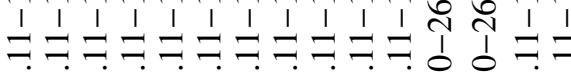
nों

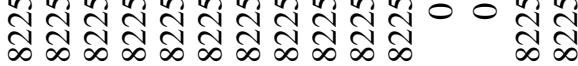

ते ते

तु तु

ते ते

तु $ु$ तु

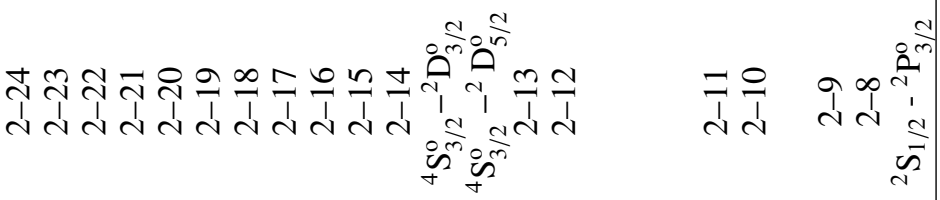

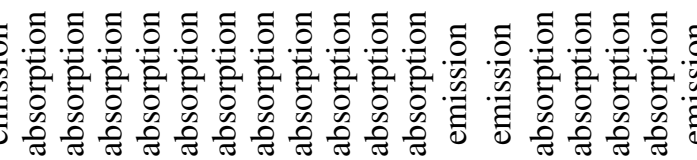

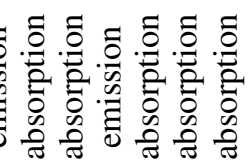

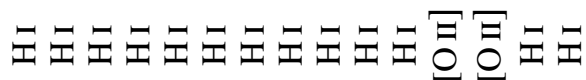

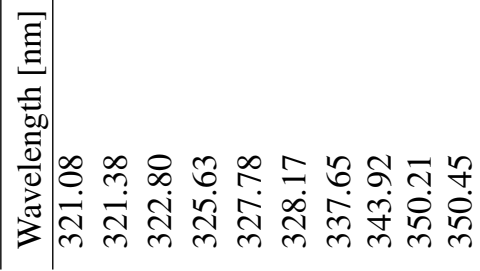

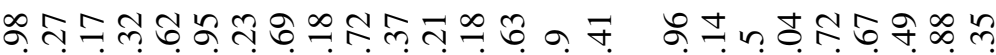

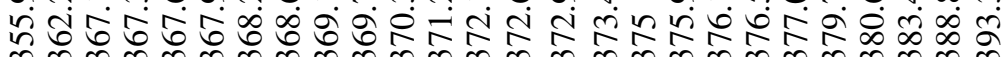

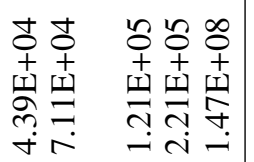
㞋王王寻

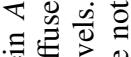

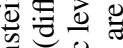

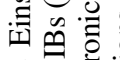

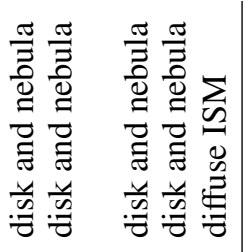

से

소 ㅎํㅇำ

कó

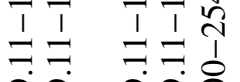

公合

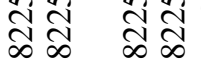

○ิ

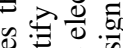

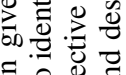

青穴造

ठํ.

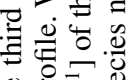

हैं क्ष

$\dot{0}$ 过

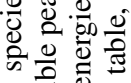

흉 छ

형

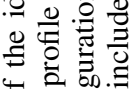

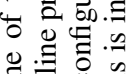

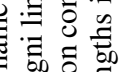

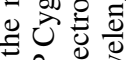

긍ㅊㅎ

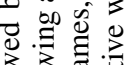

응 芯

क ज्ञ

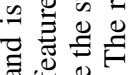

घं की

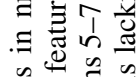

营.

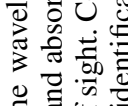
छ 荘

ڤ. 유

कo.

声

究造

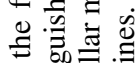

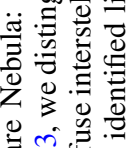

壳

次

\& 00.

$\Xi$ 节

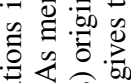
氖的泀 


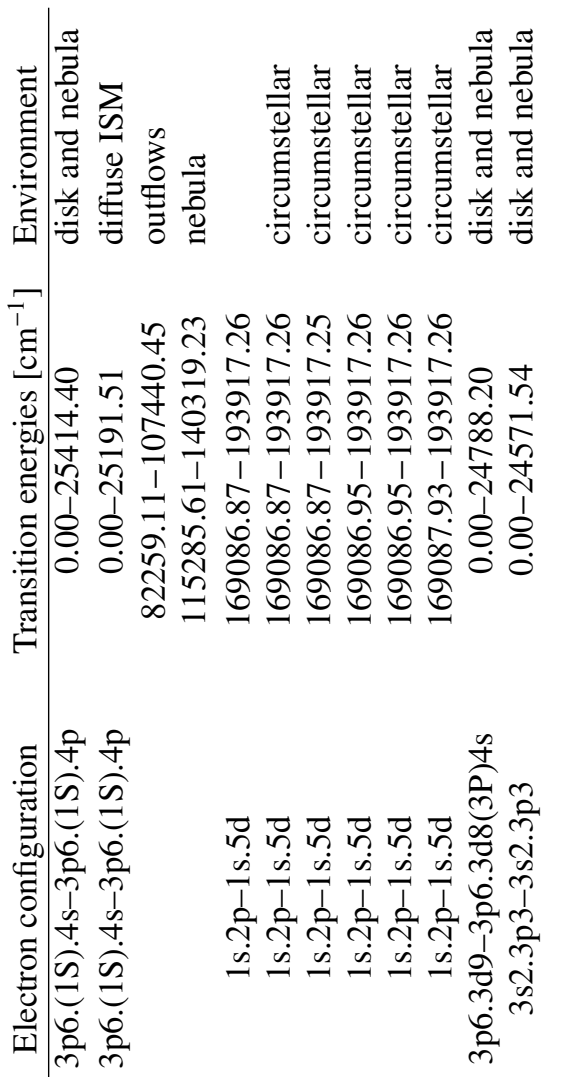

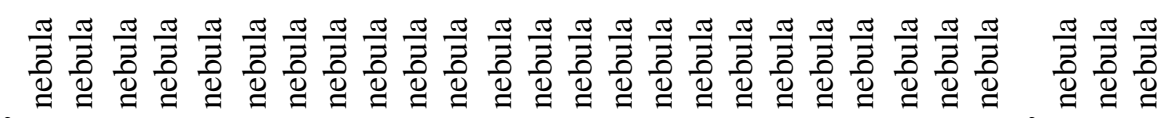

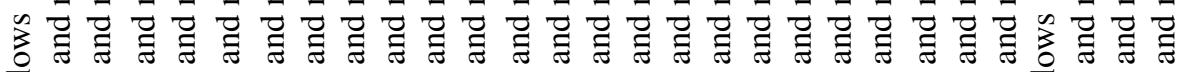

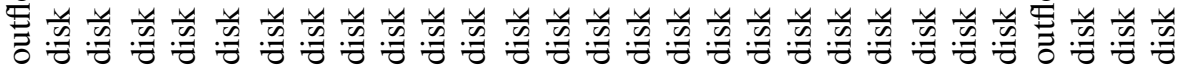

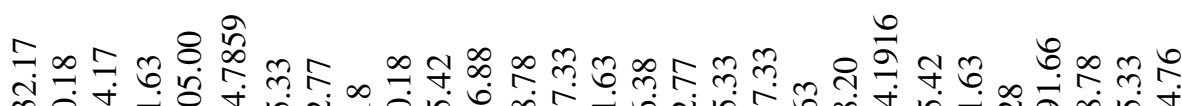

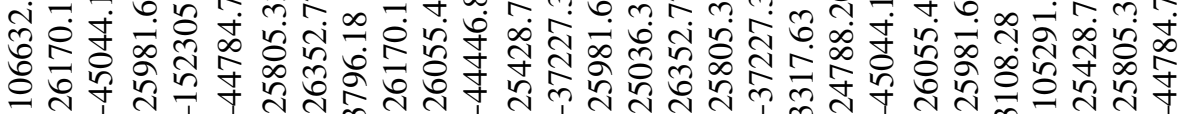

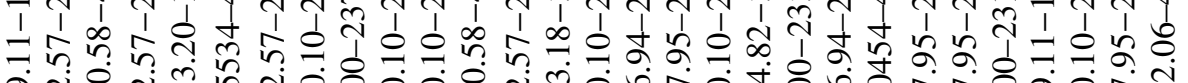

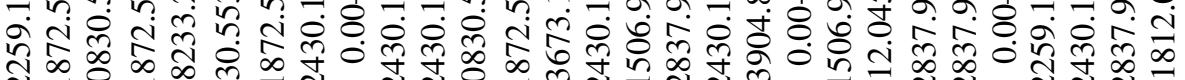

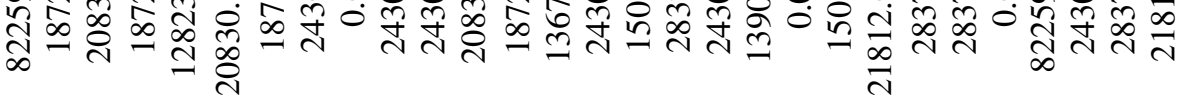

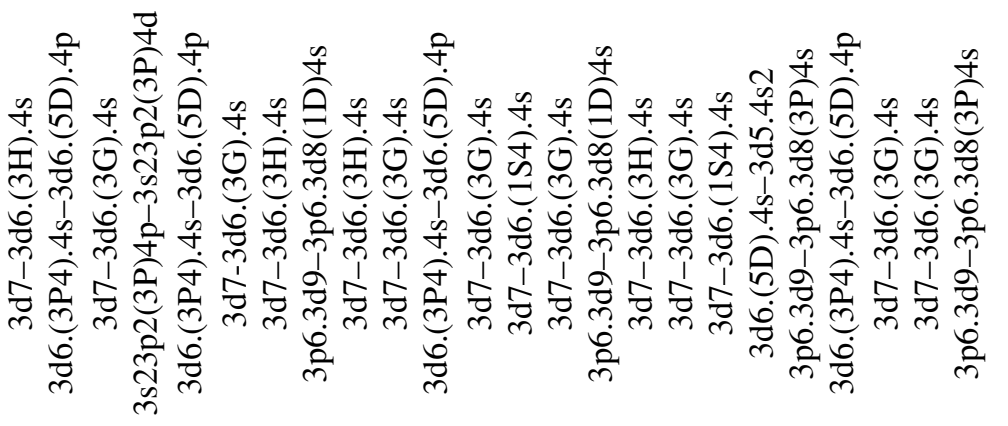

:

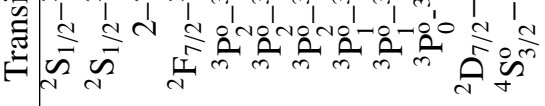

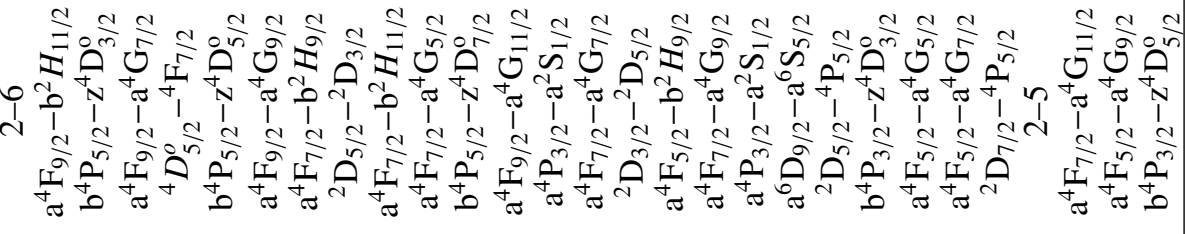

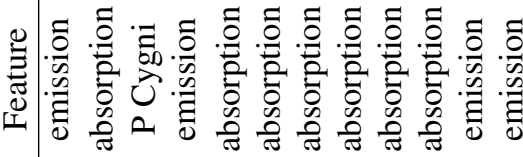

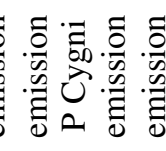

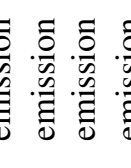

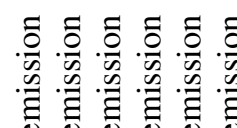

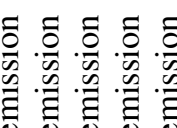

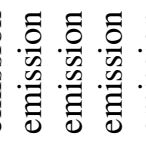

$T_{0}$ 凹

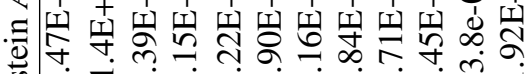

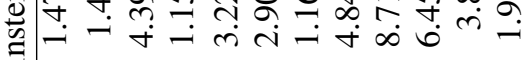

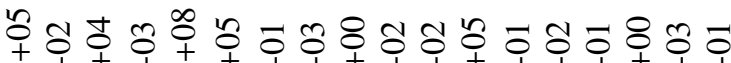

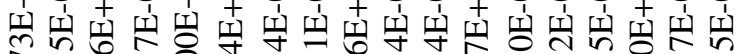
å

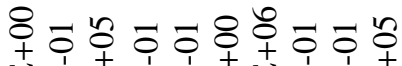

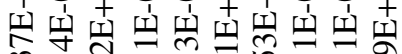

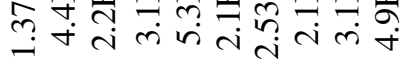

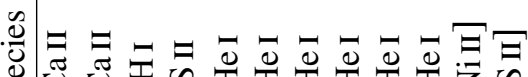

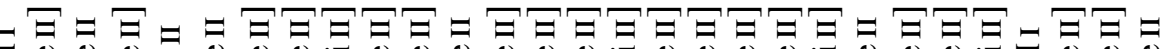

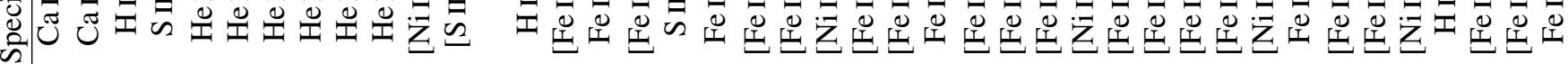

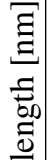

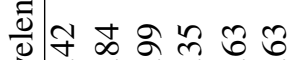

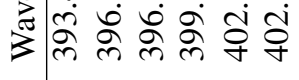

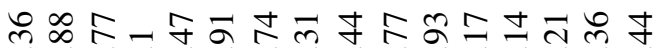

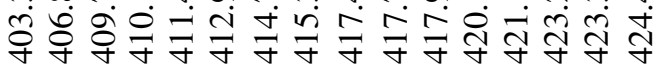

a느소

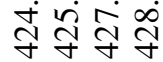

于 ले สู $85 \pi m$

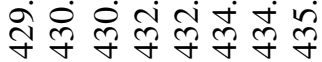




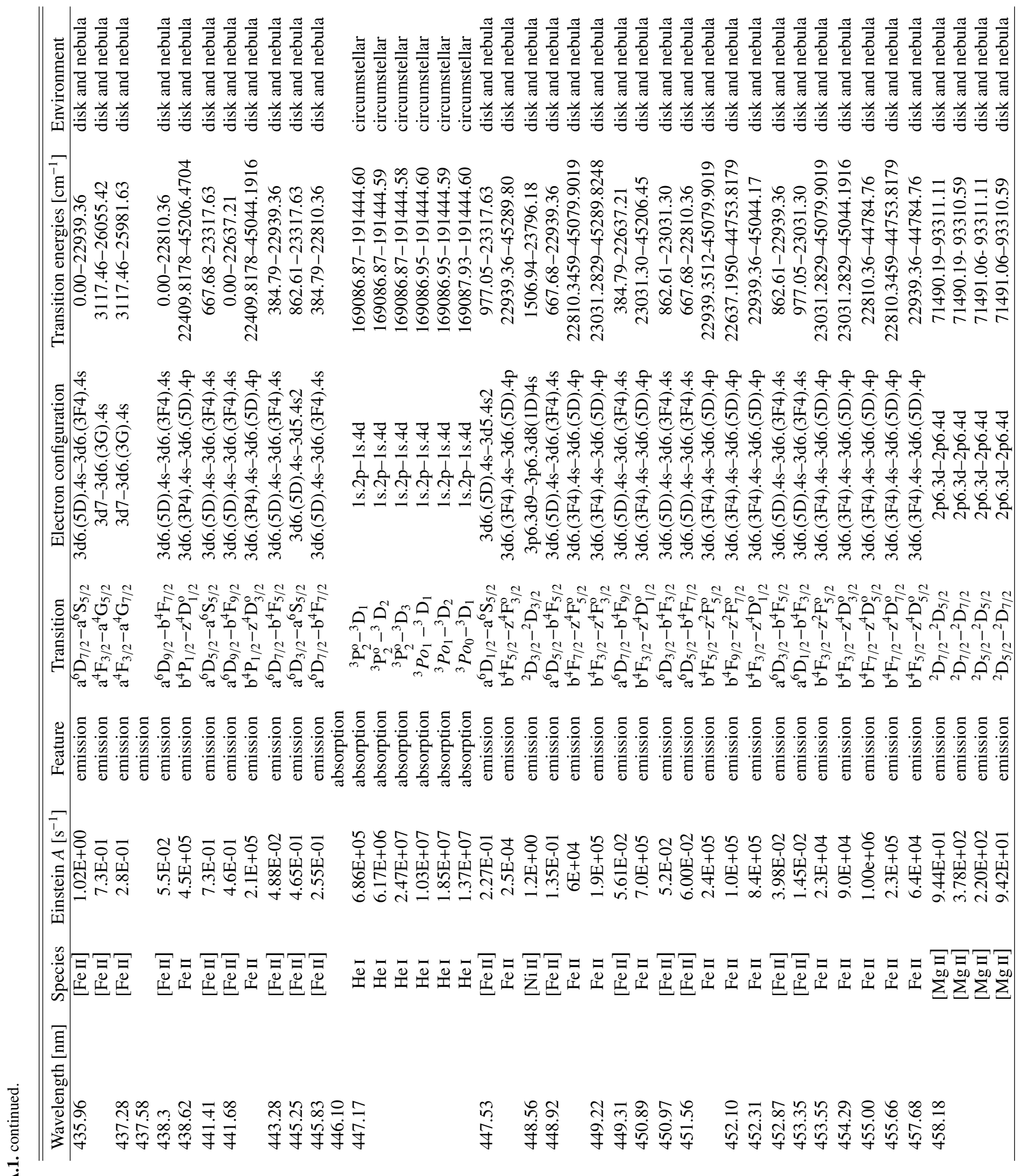




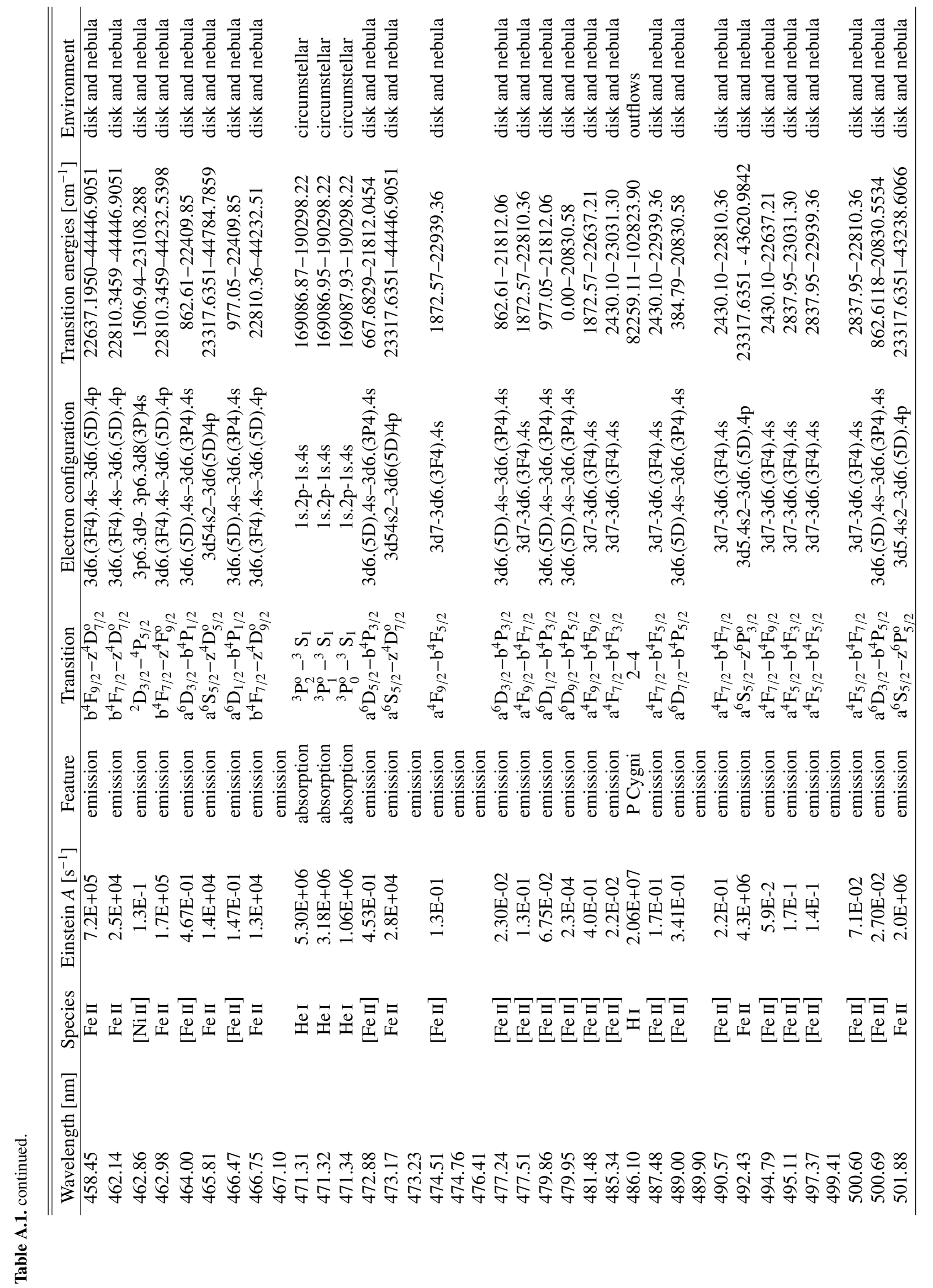




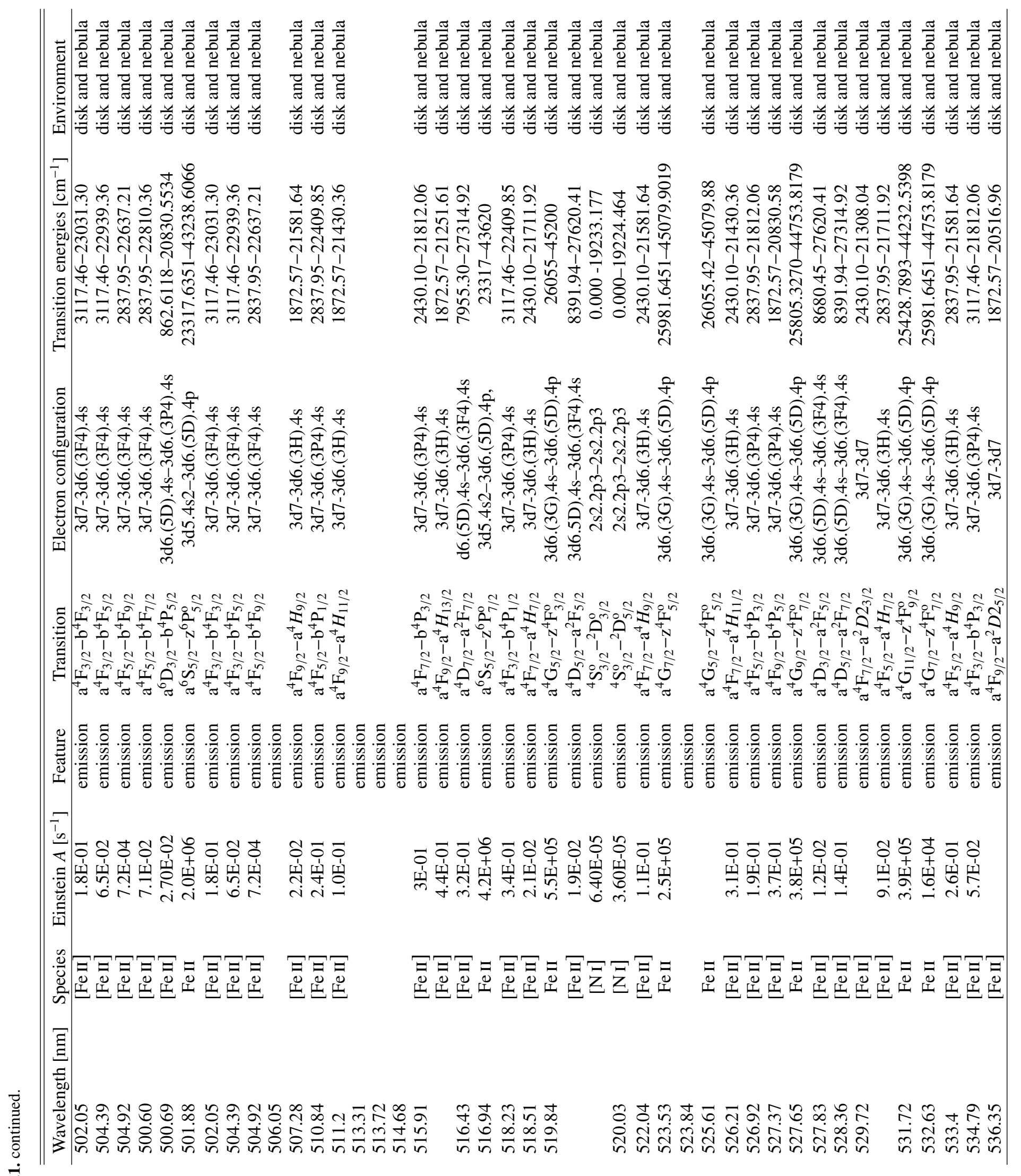




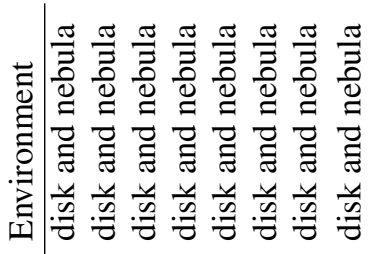

Тี่

娄

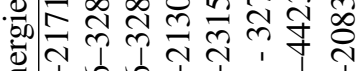

ฮ.

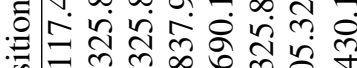

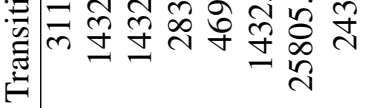

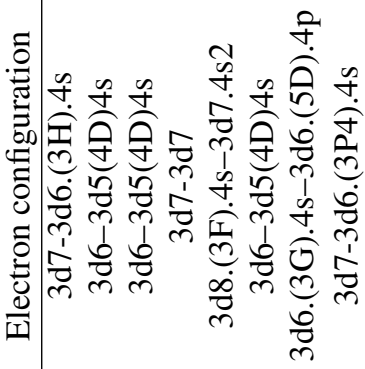

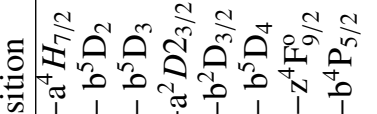

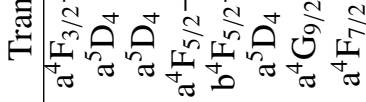

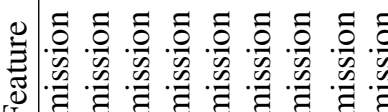

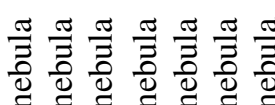

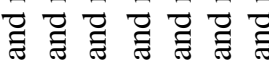

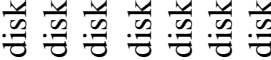

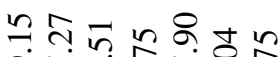

ठิ

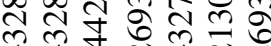

in 1 i 1 i T 1

कळ

ர்

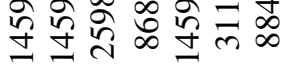

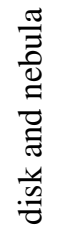

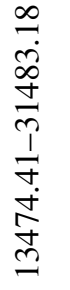

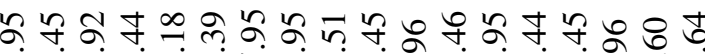

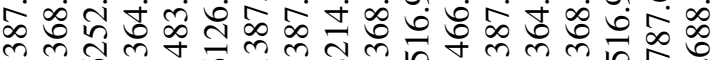

m m d্

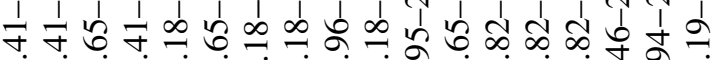

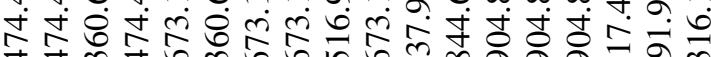

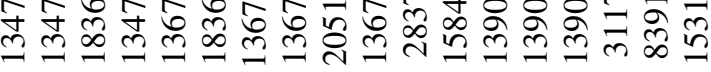

กิ 누ำ 8 +

守过它

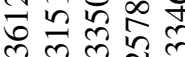

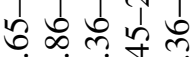

จํ.

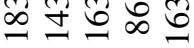

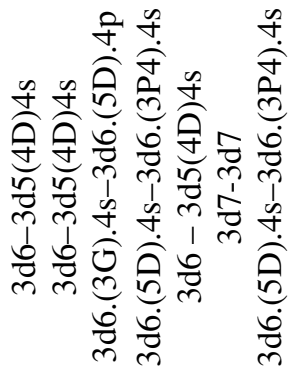

$$
\text { 今ิ }
$$

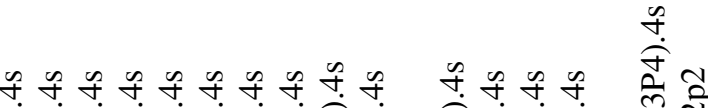

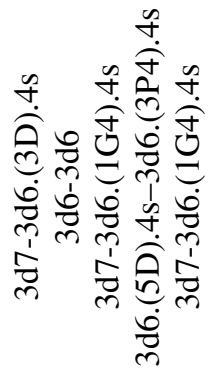

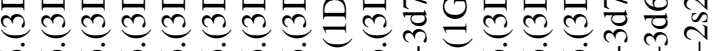

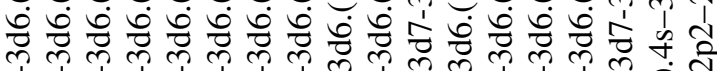

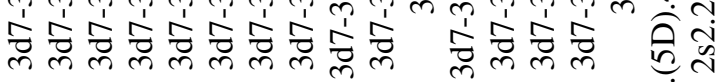
कृ
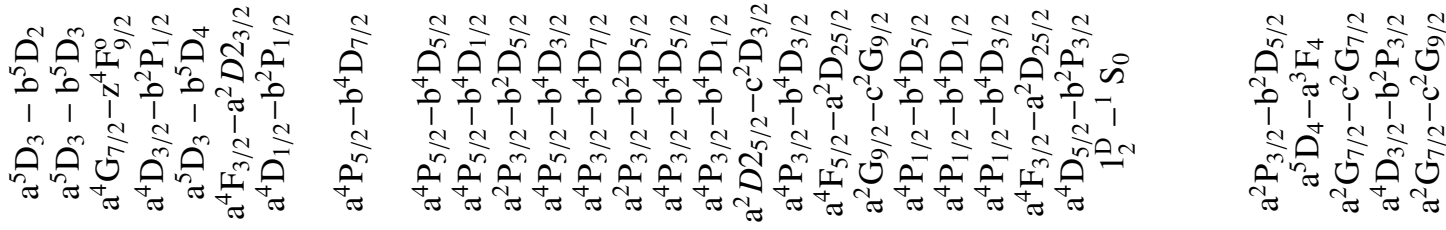

\section{ฮี .ี

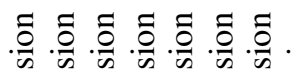 \\ ธี .․ำ. .ิ \\ .르.

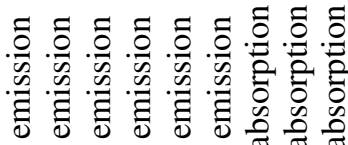

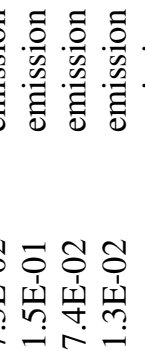

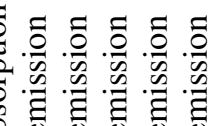

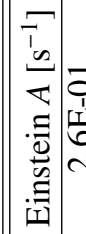

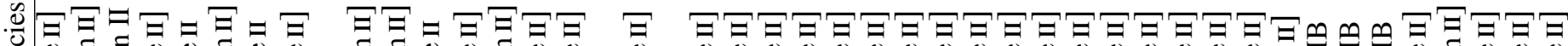

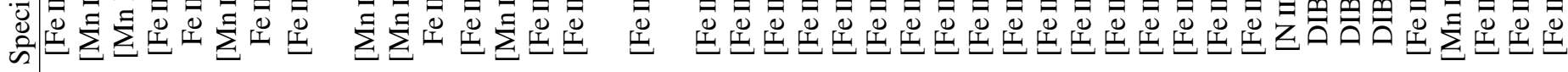

$\bar{\Xi}$

䍃

可

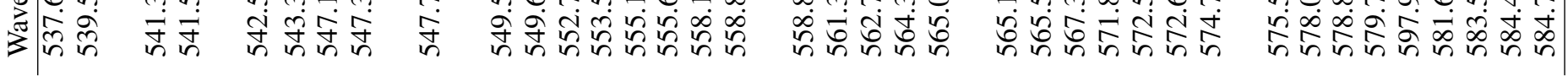




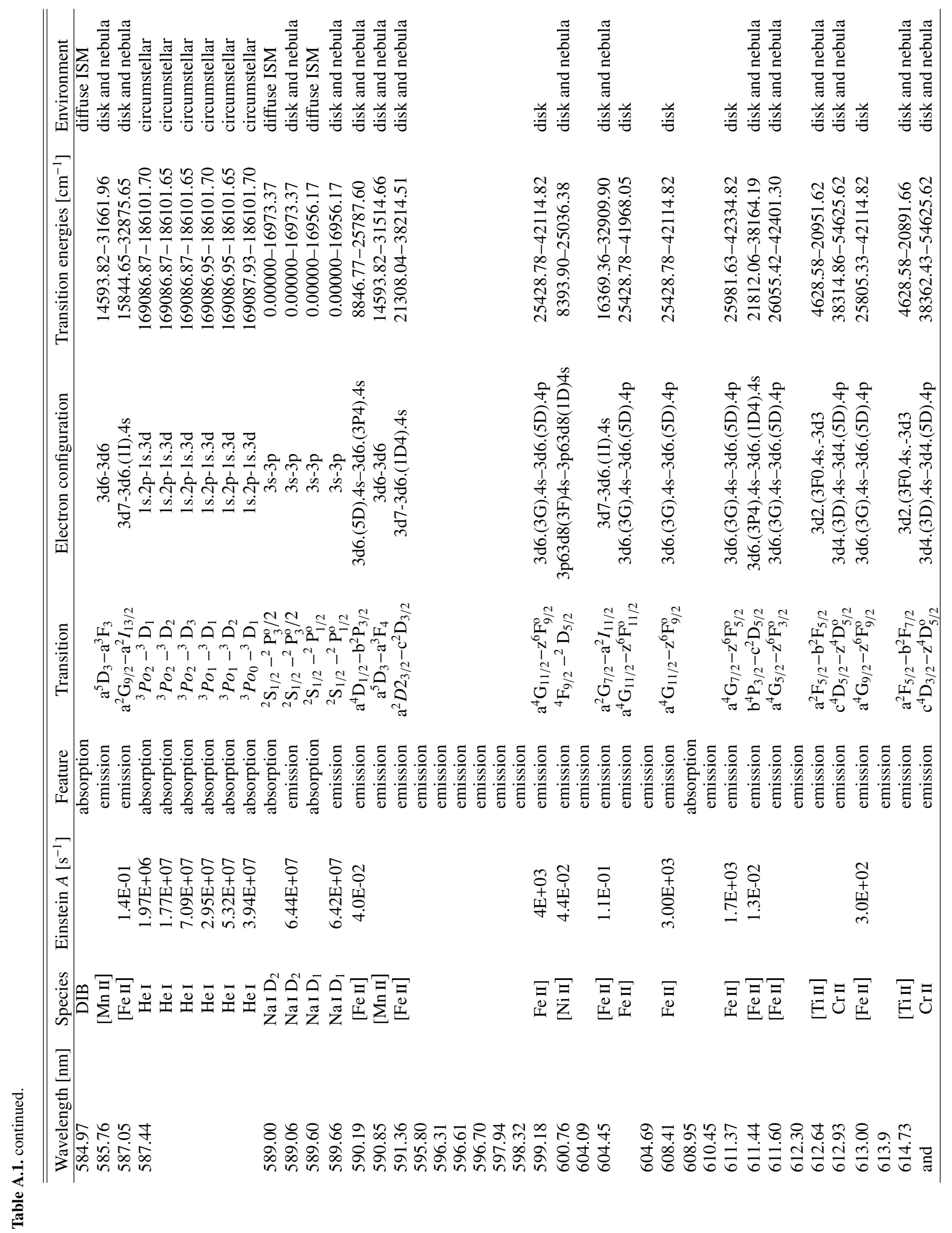




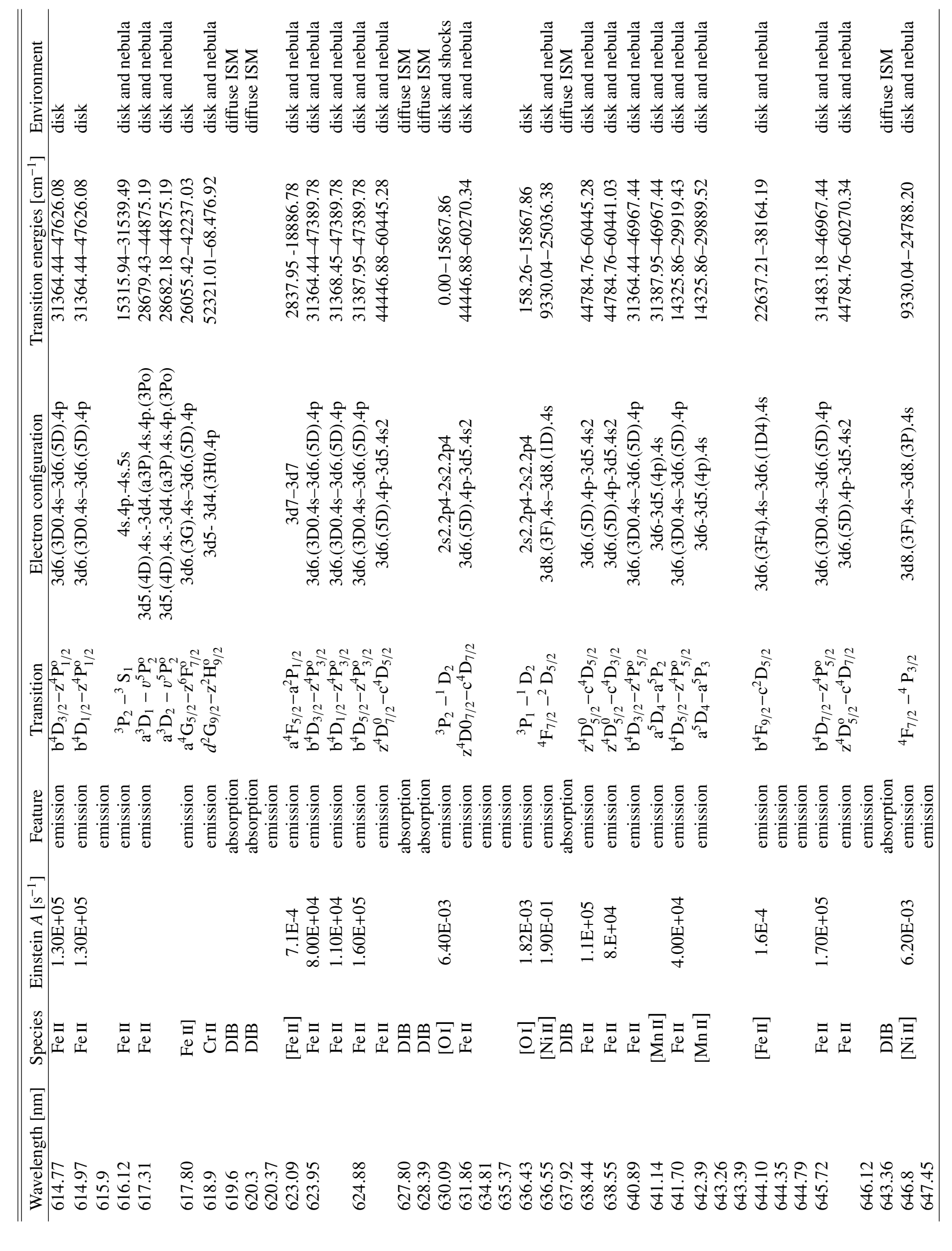




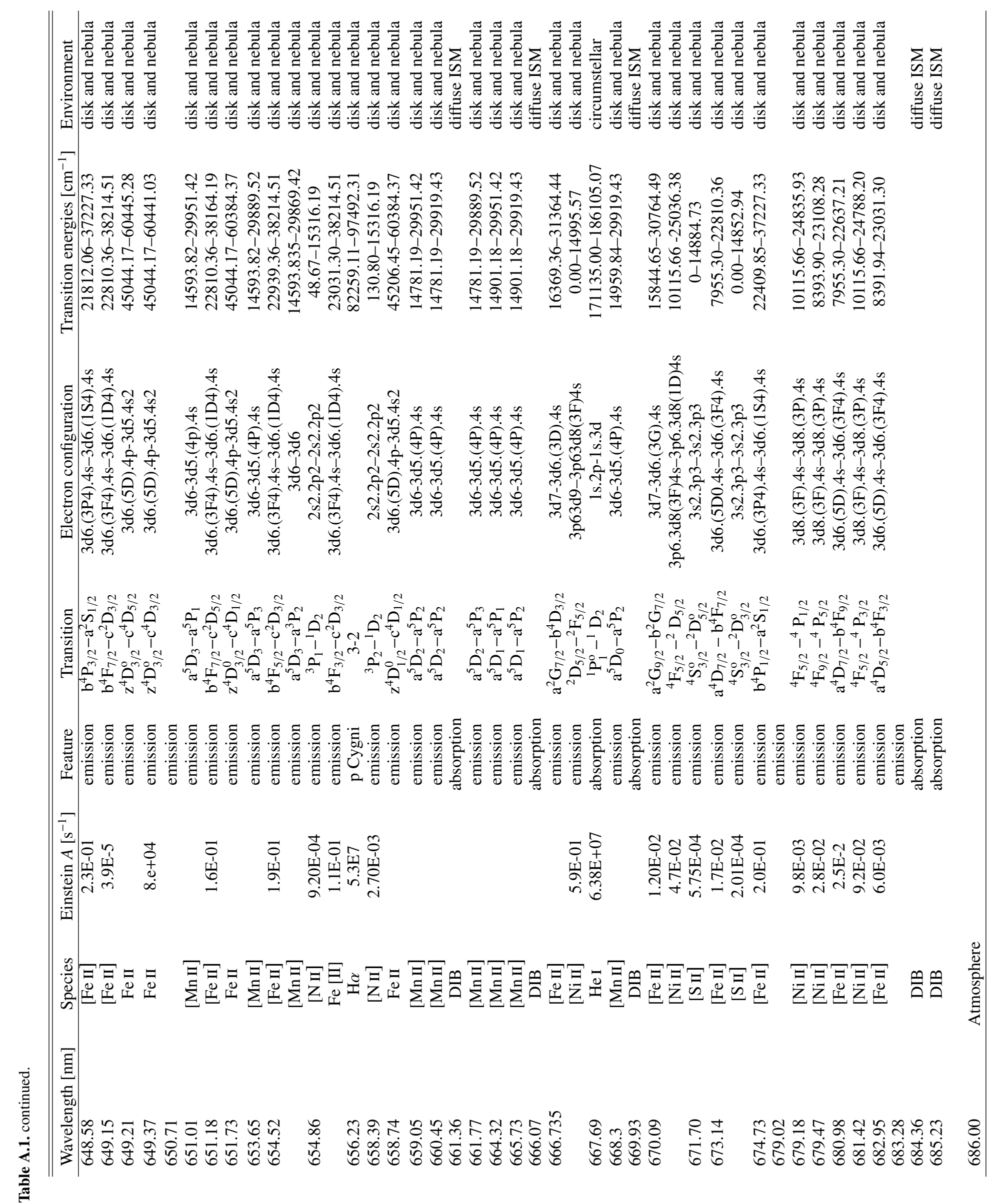




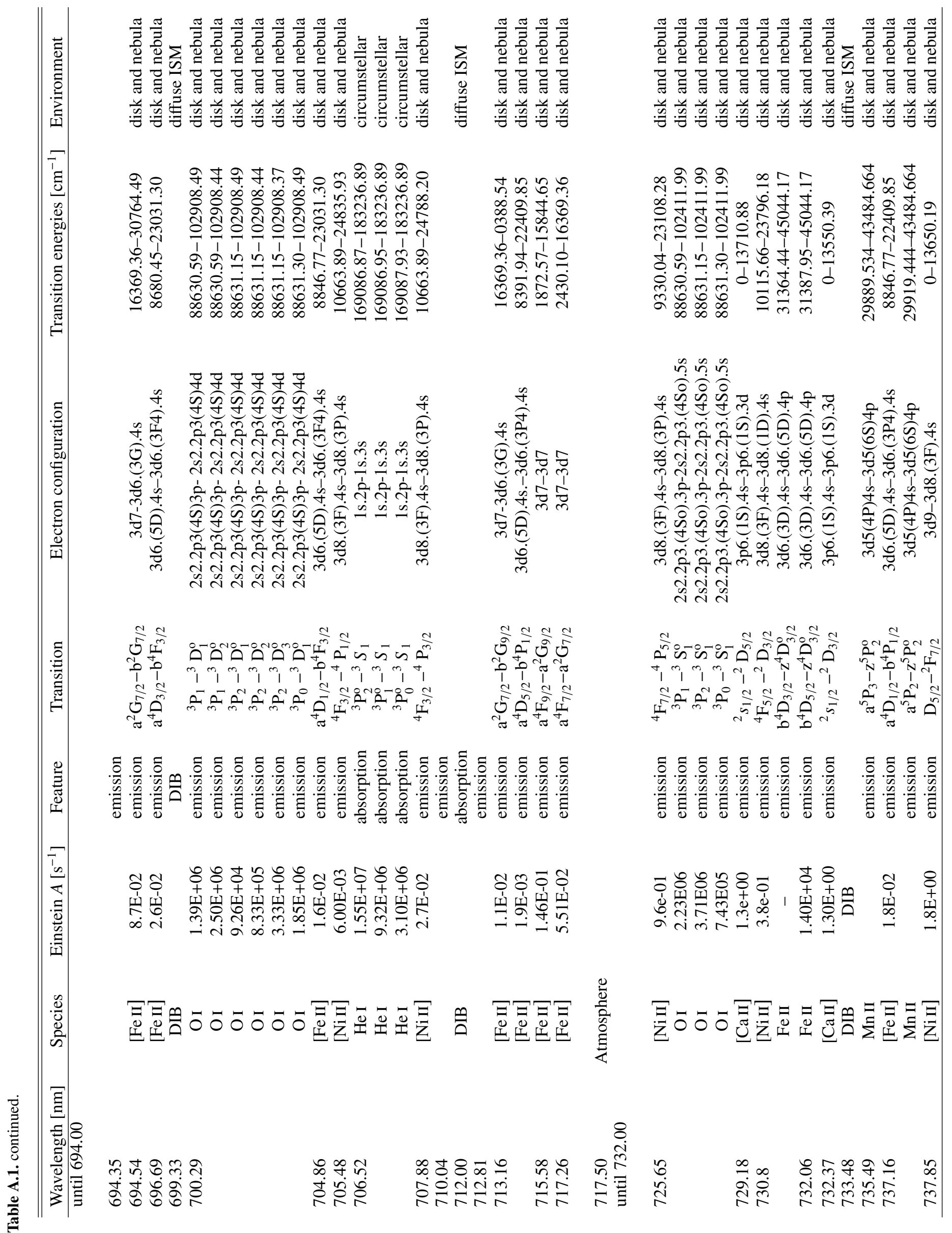




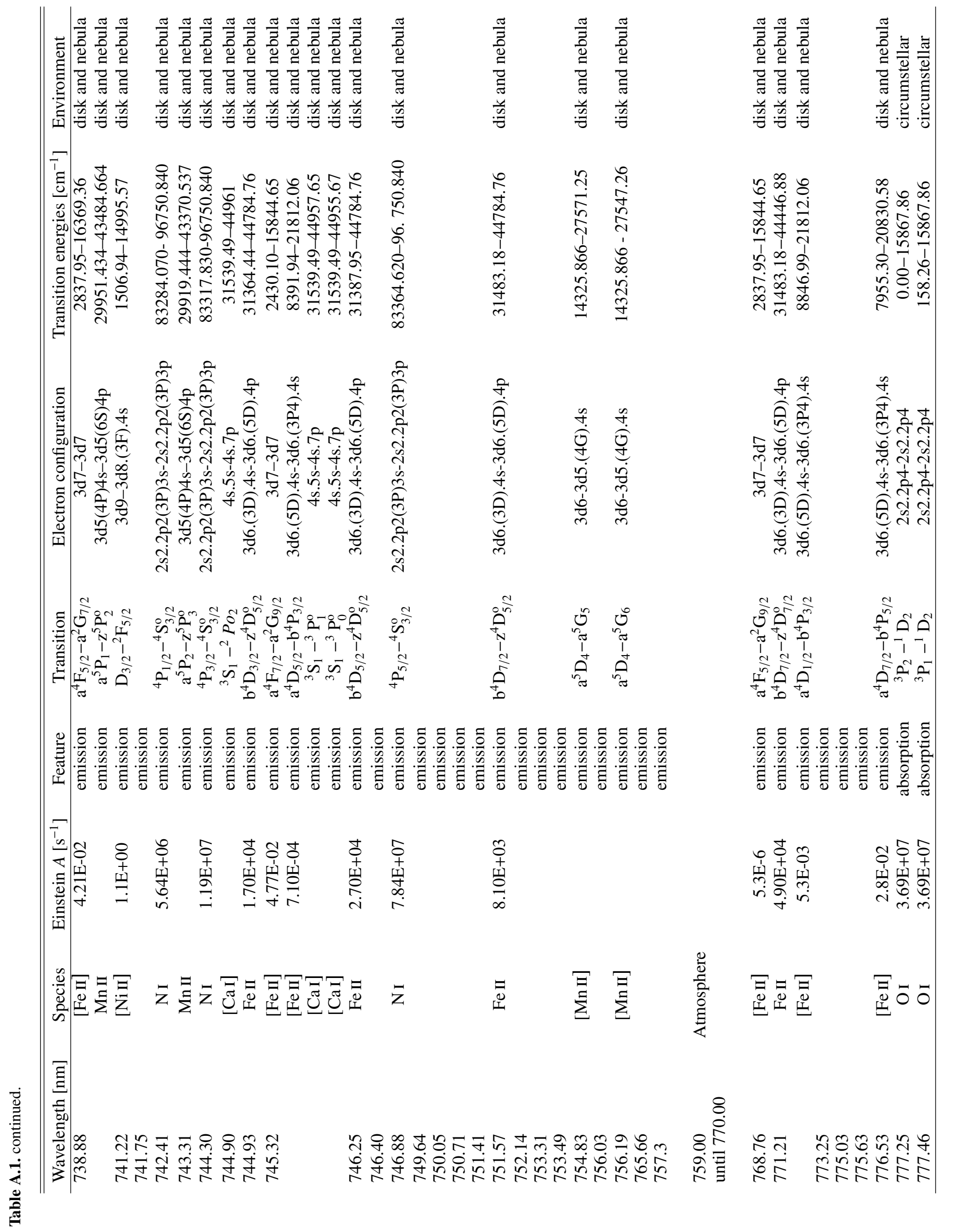




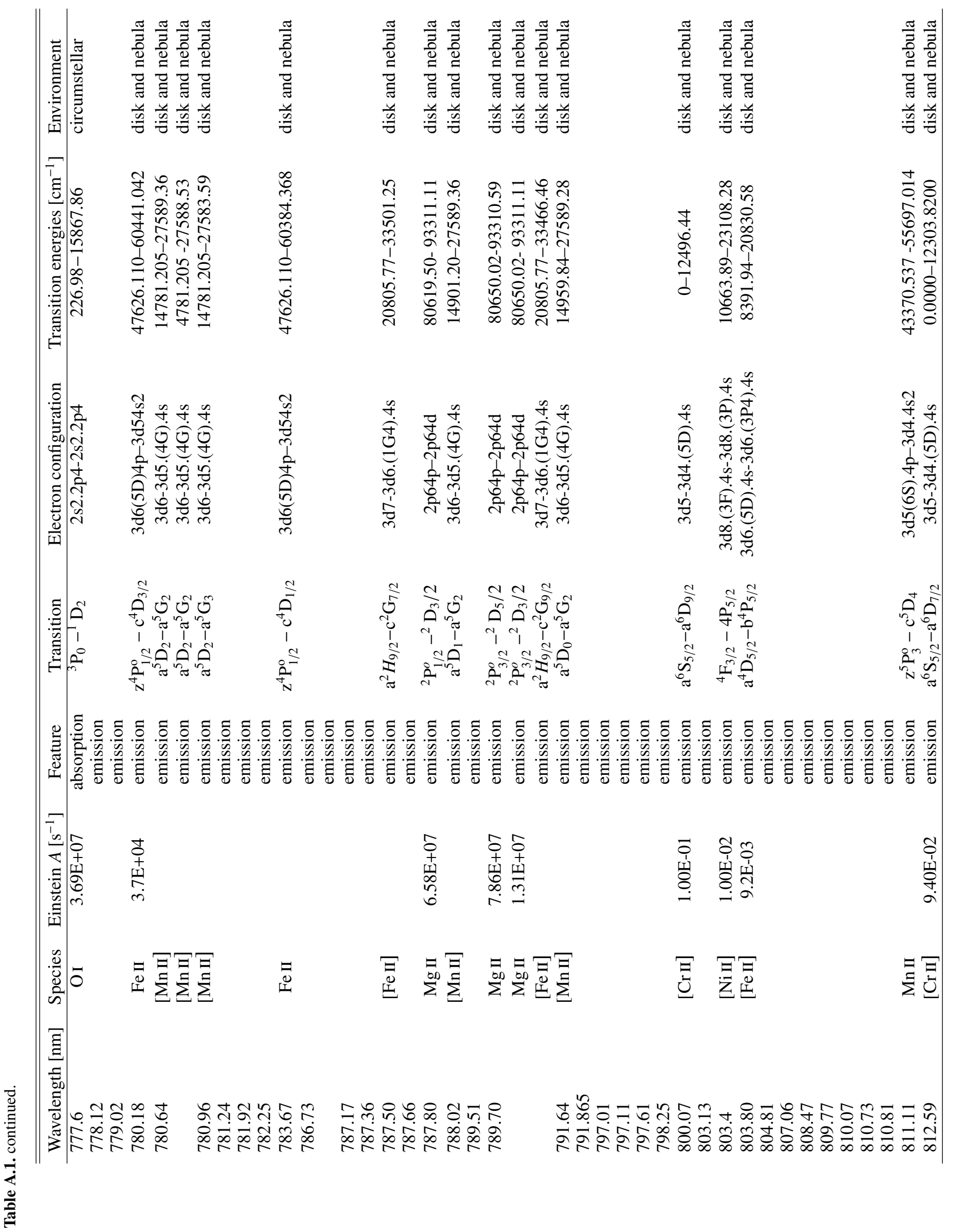




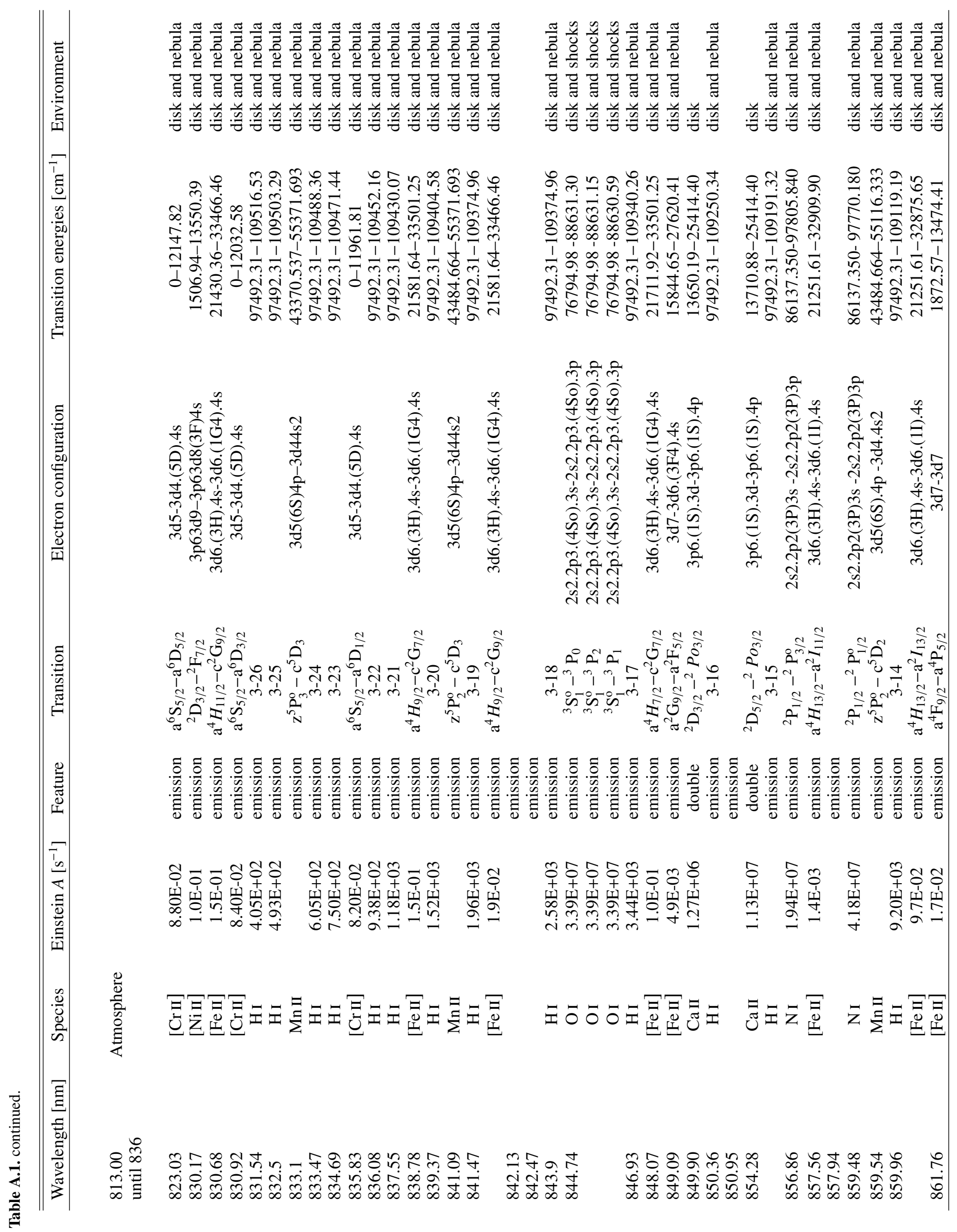




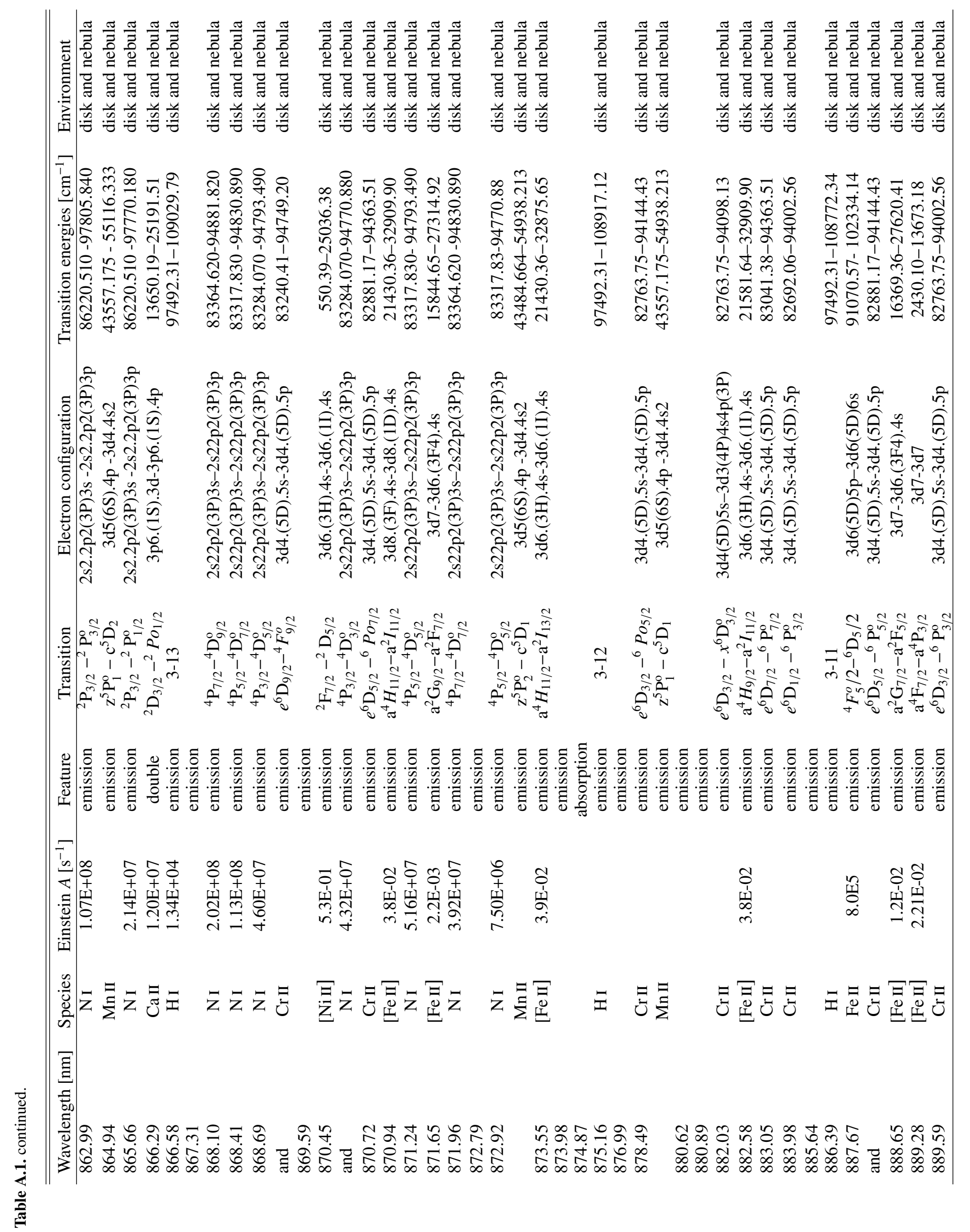




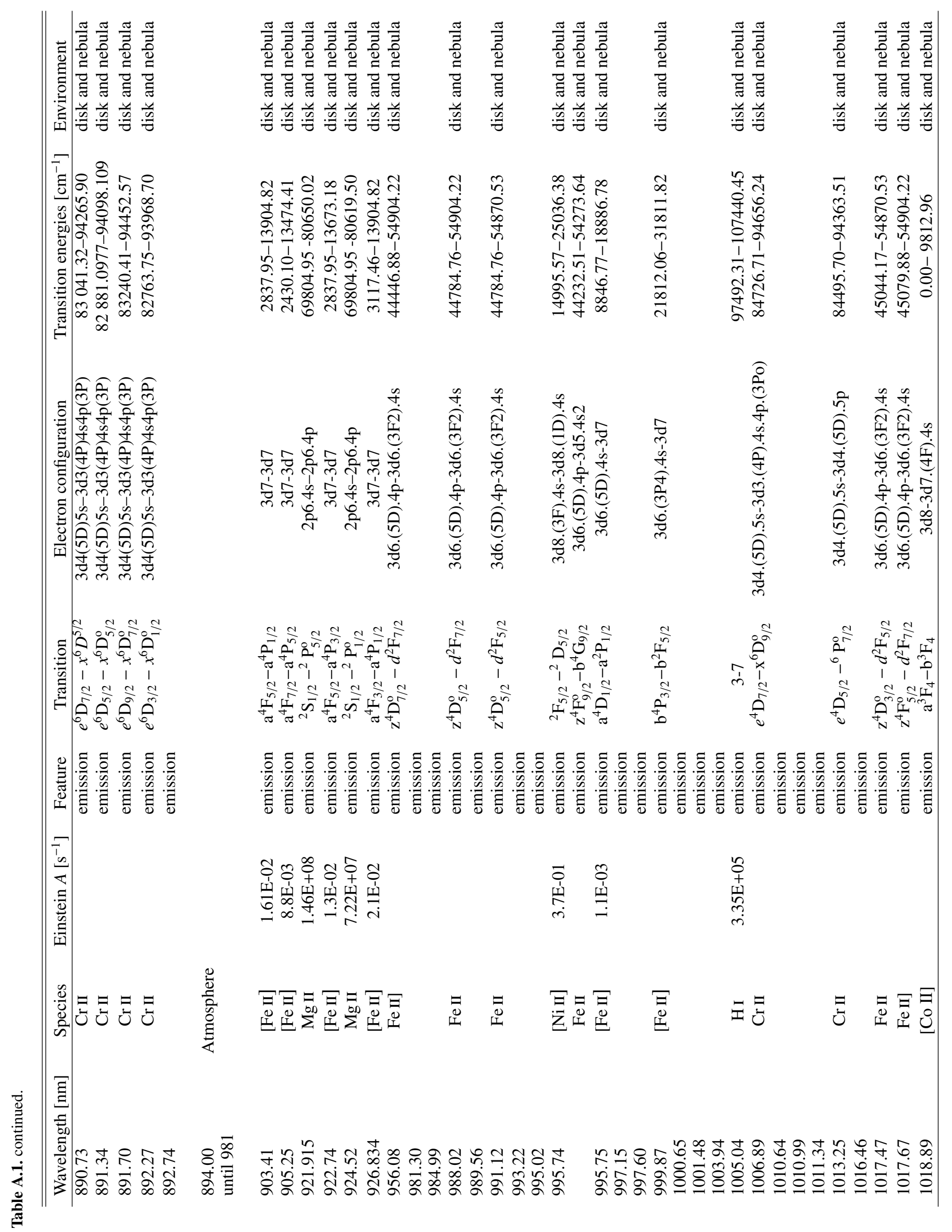




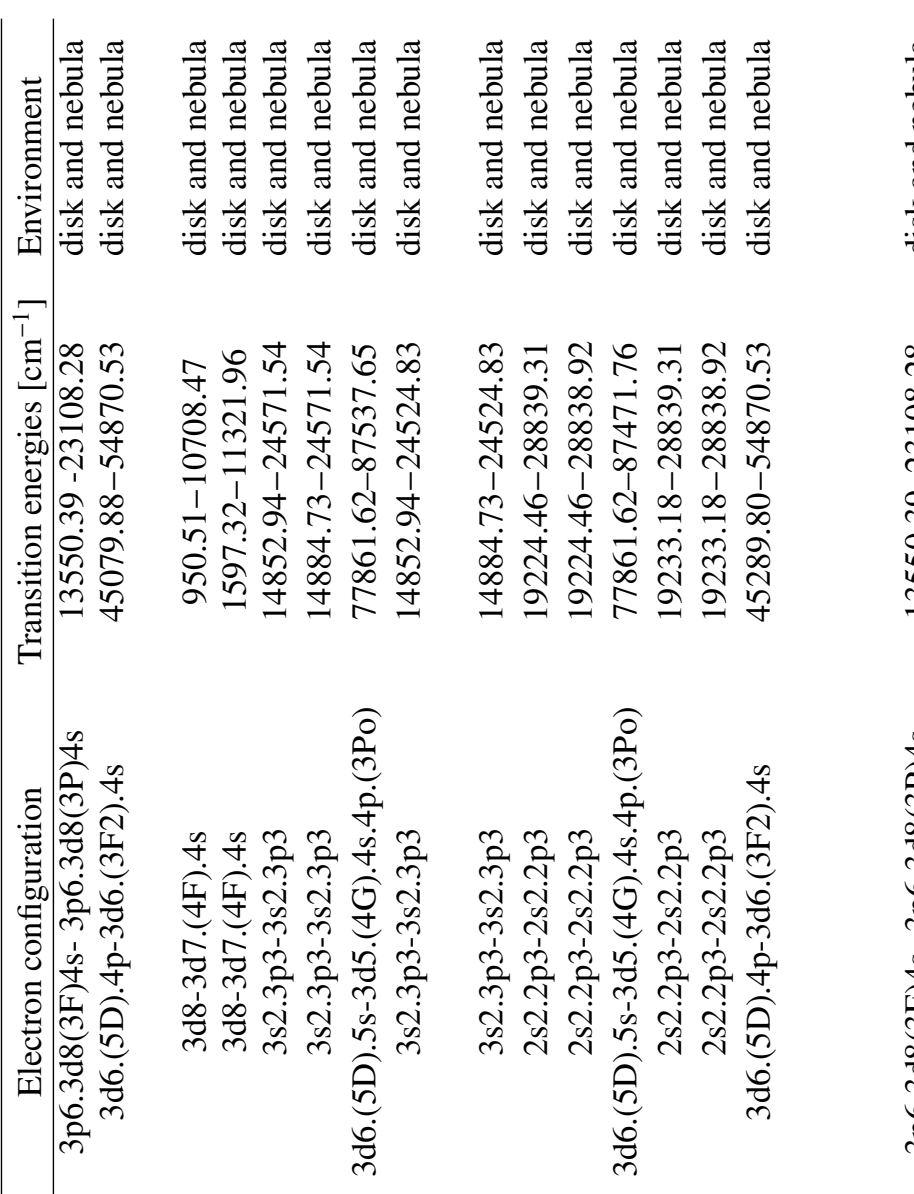

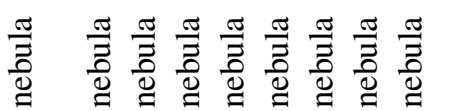

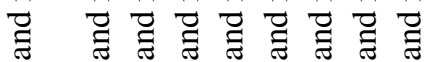

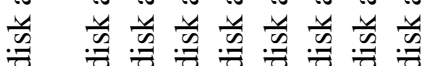

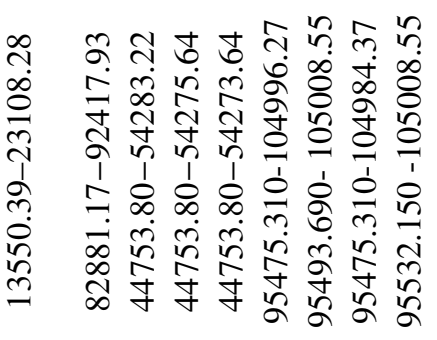

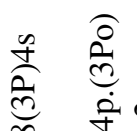

官

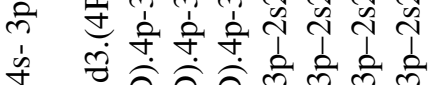

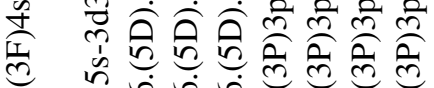

की
말 ले ले ले
家

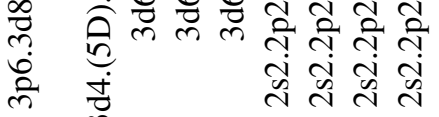

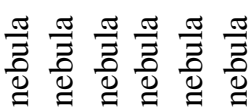

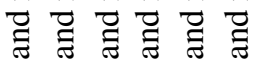

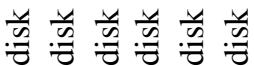

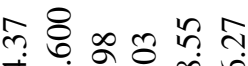

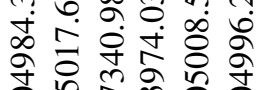

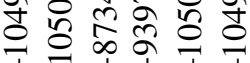

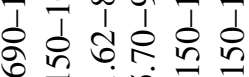

政

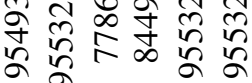

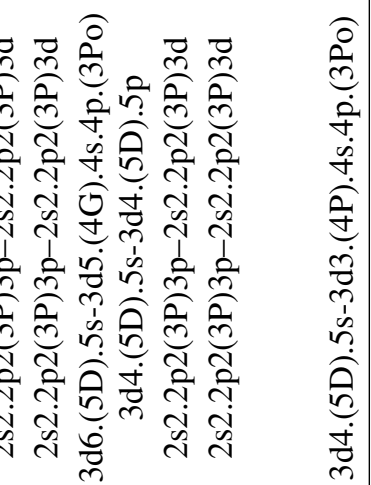

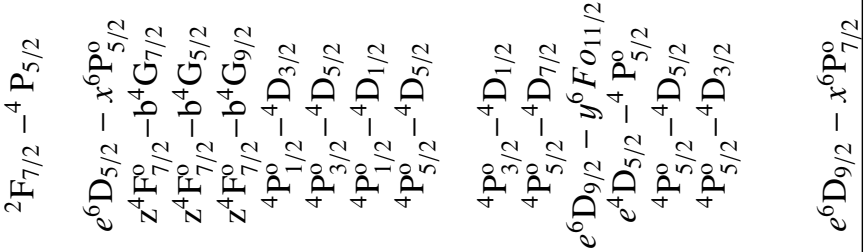

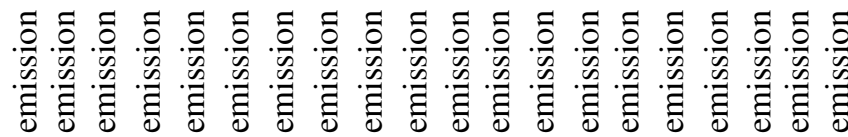

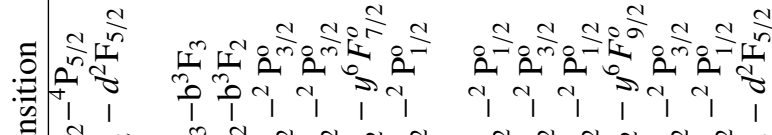

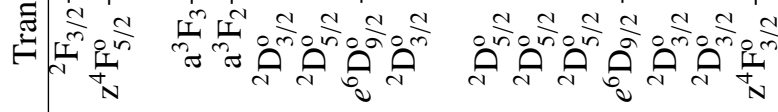

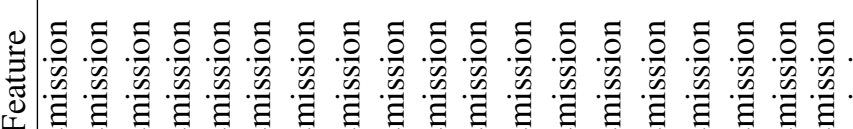

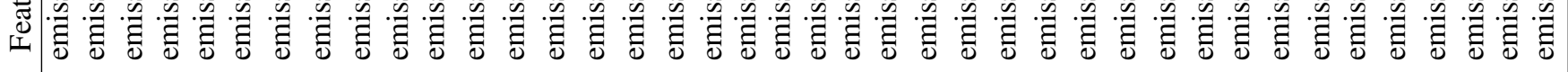

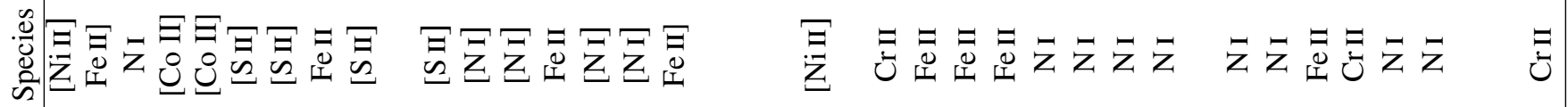

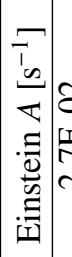

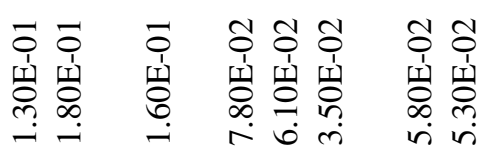

至

五

章

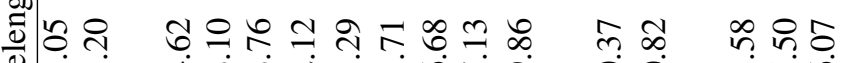

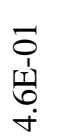

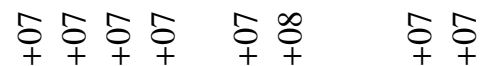

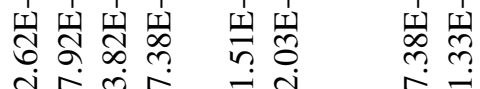

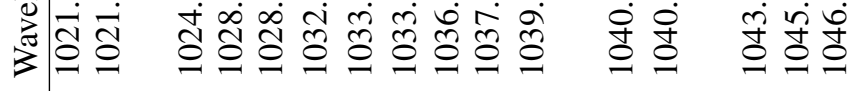

tid 守守守

舟

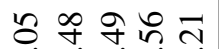




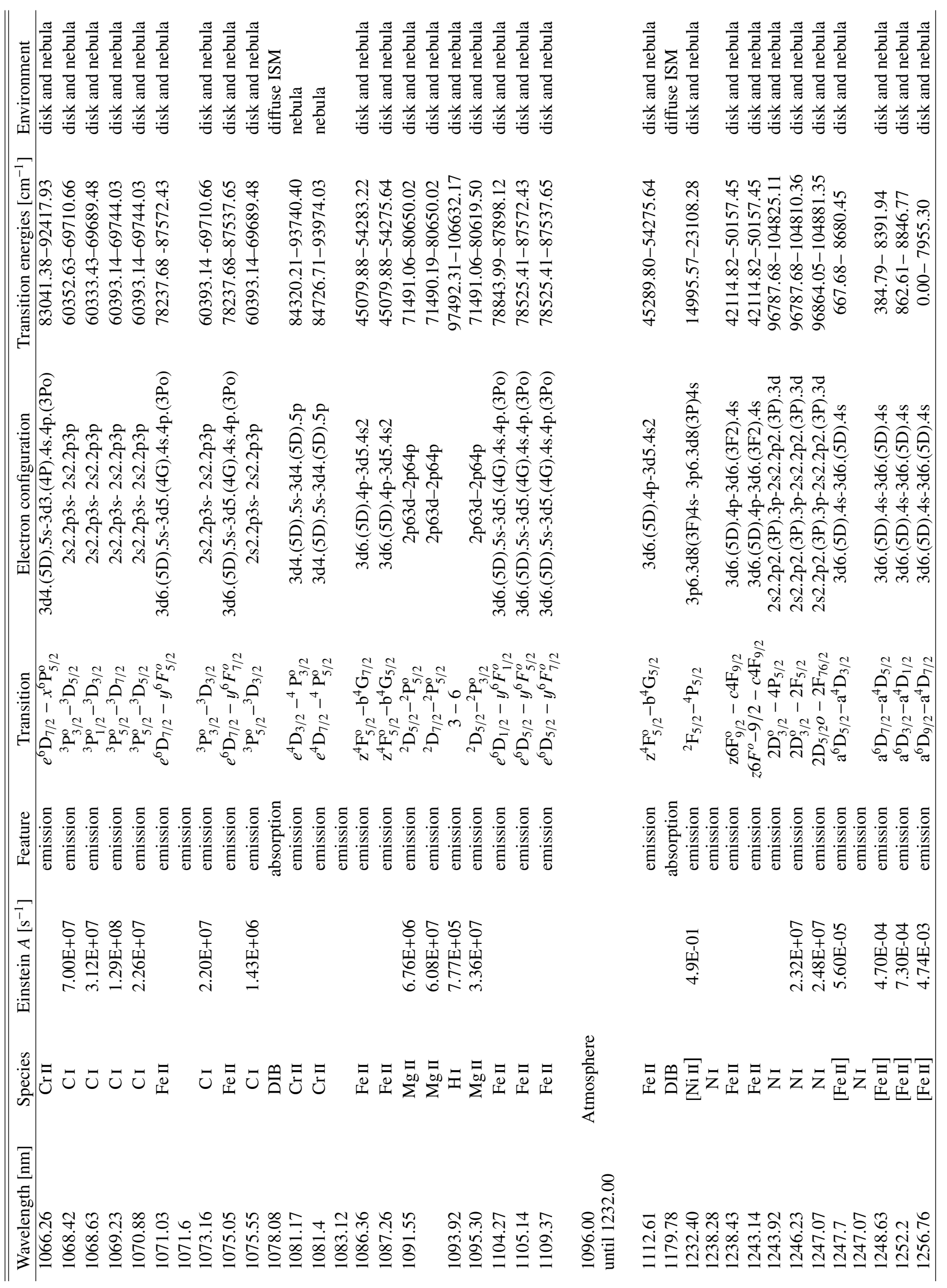




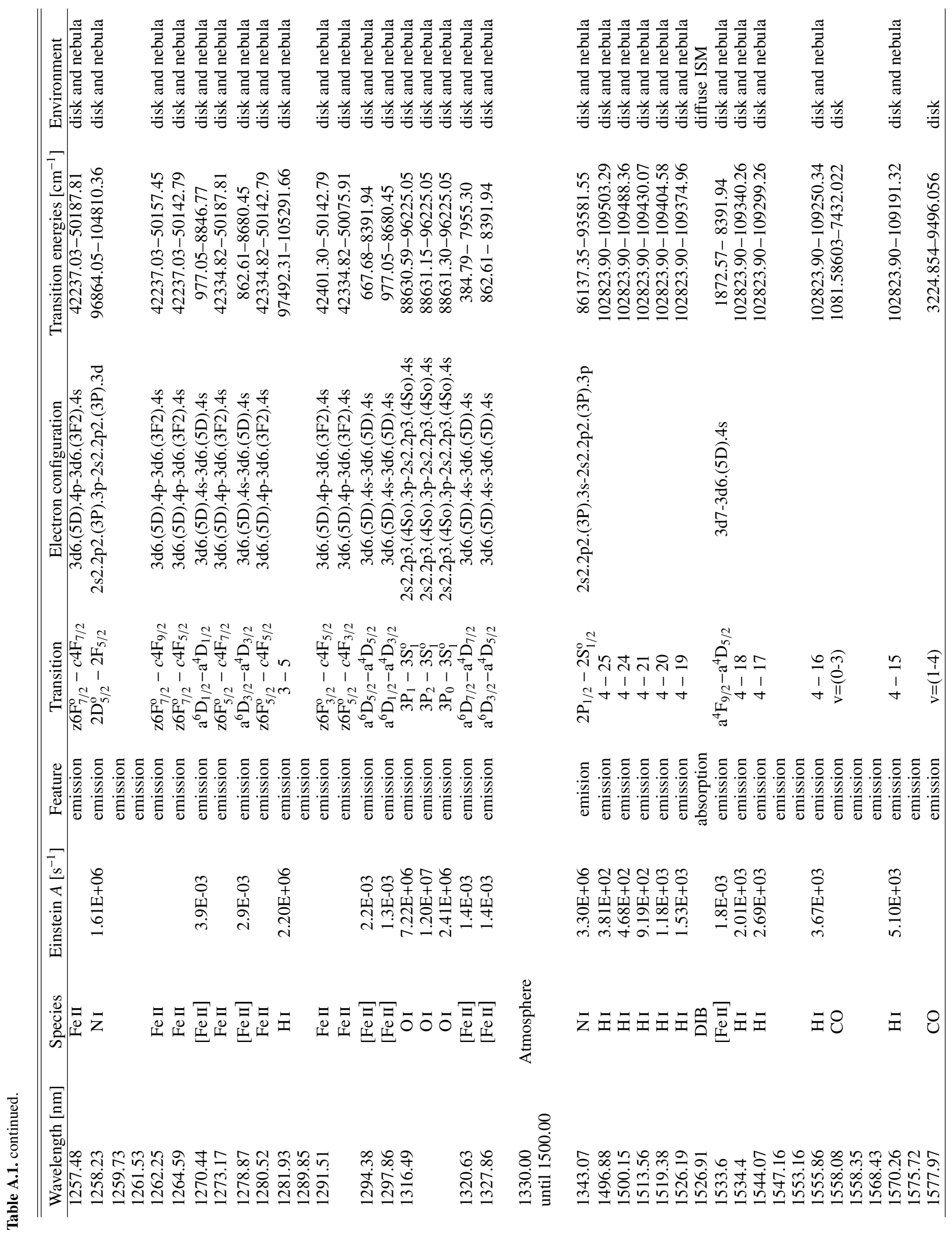




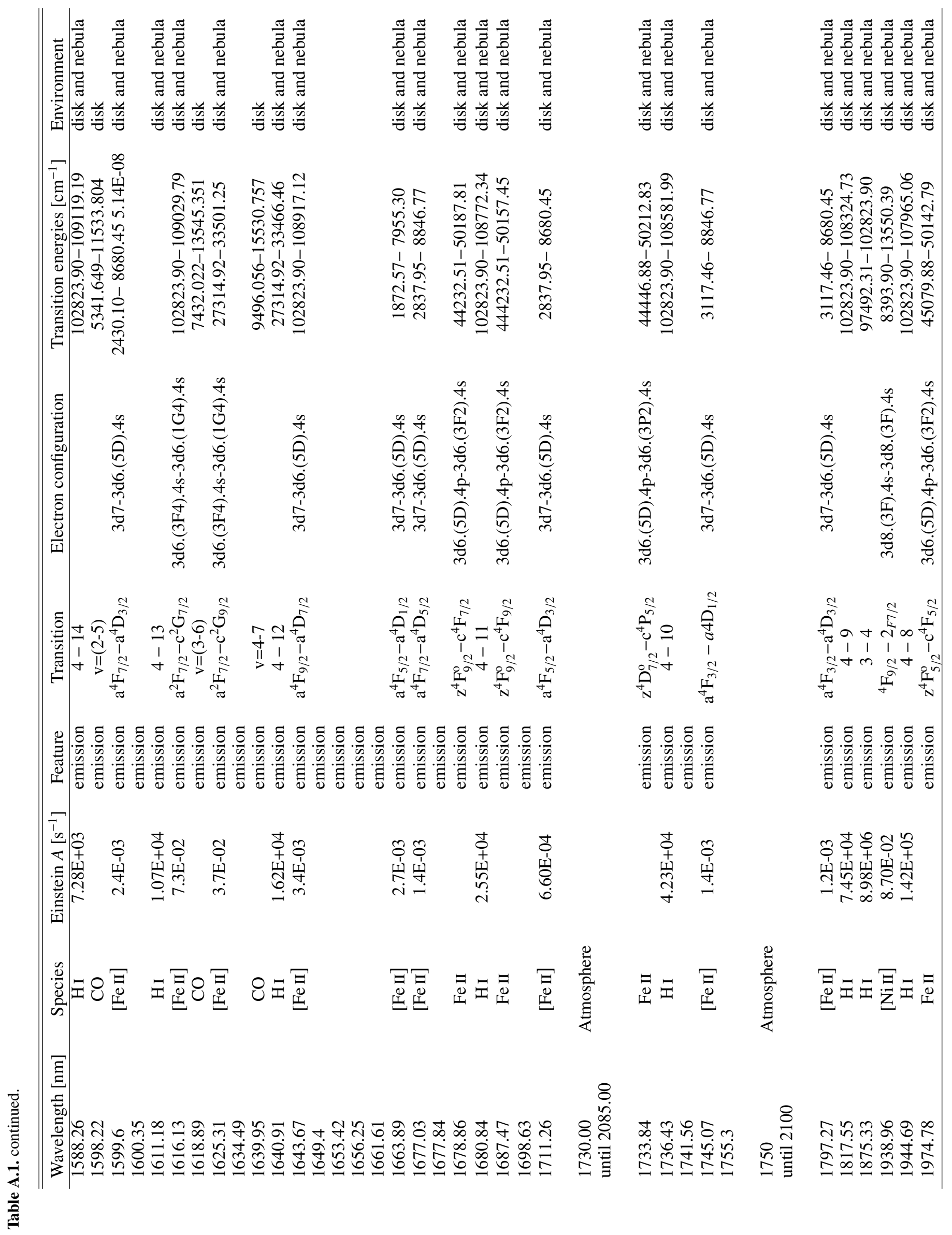




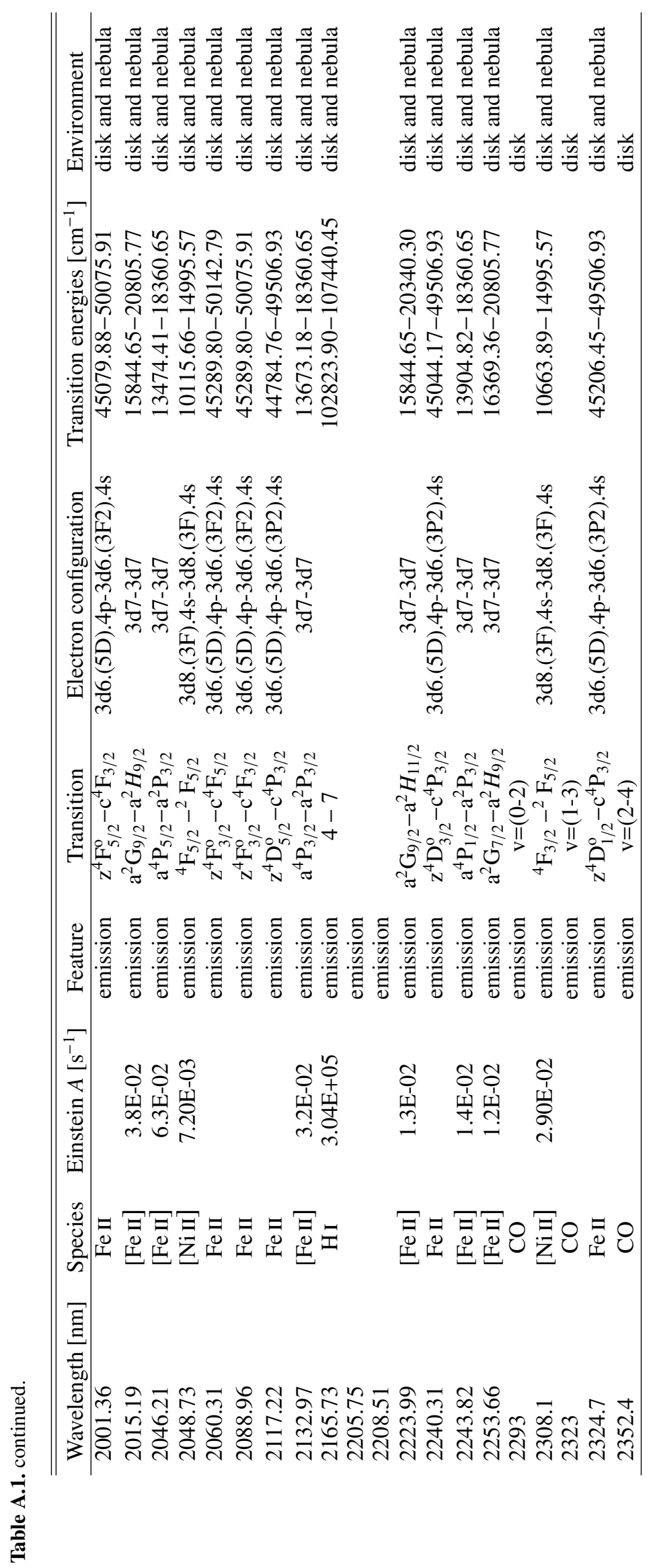

